\title{
10th Laser Display and Lighting Conference: Abstract Book
}

, "10th Laser Display and Lighting Conference: Abstract Book," Proc. SPIE 11851, 10th Laser Display and Lighting Conference (LDC 2021), 1185101 (30 June 2021); doi: 10.1117/12.2601617

SPIE. Event: Laser Display and Lighting Conference 2021, 2021, Online Only 


\section{LDC 2021 Proceedings}

\section{Laser Display and Lighting Conference 2021}

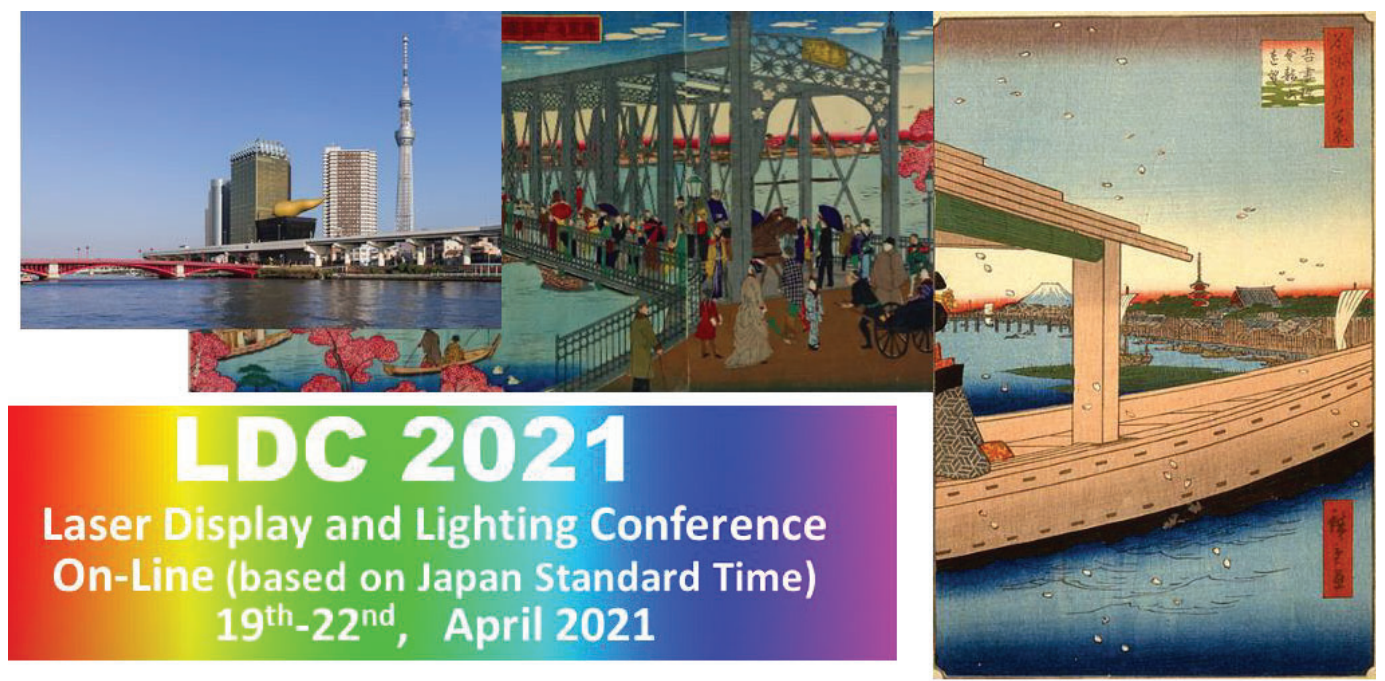




\section{Laser Display and Lighting Conference 2021}

\section{Introduction}

The Laser Display and Lighting Conference (LDC) is an international conference on laser displays, laser lighting, and related technologies. The 1st, 2nd, 4th, and 6th-8th LDC were held in Yokohama, Japan in 2012, 2013, 2015, 2017-2019 respectively, and the 3rd in Taichung, Taiwan in 2014, the 5th in Jena, Germany in 2016. The 9th LDC was held online. The 10th Laser Display and Lighting Conference (LDC2021) will be held online as well.

The LDC2021 is intended to provide a central forum for the update and review of scientific and technical information on laser display and lighting covering a wide range of fields from fundamental research to systems and applications. The conference is sponsored by the Optical Society of Japan in cooperation with several academic societies and associations.

For details, please come to our web-site;

https://ldc.opicon.jp/

\section{Chairs}

$\begin{array}{rrl}\text { Conference chair } & \text { co-chair } & \text { Kazuo Kuroda } \\ & \text { co-chair } & \text { Hiroshi Murata } \\ & \text { co-chair } & \text { Fergal Shevlin } \\ \text { Steering committee } & \text { co-chair } & \text { Kazuhisa Yamamoto } \\ & \text { co-chair } & \text { Norihiro Ohse } \\ \text { Program committee } & \text { co-chair } & \text { Sunao Kurimura } \\ & \text { co-chair } & \text { Tetsuya Yagi } \\ & \text { co-chair } & \text { Shining Zhu } \\ \text { vice co-chair } & \text { Tatsushi Hamaguchi } \\ \text { vice co-chair } & \text { Muneharu Kuwata } \\ \text { vice co-chair } & \text { Hirotsugu Yamamoto }\end{array}$


Sponsors

Sponsored by

The Optical Society of Japan

Co-Sponsored by

The Japan Society of Applied Physics

Organized by

Laser Display Technology Group (LDT) of Optical Society of Japan (OSJ)

In cooperation with

SPIE,

the Chemical Society of Japan,

the Society of Polymer Science, Japan,

International Display Workshop

Information Photonics

The Laser Society of Japan

Consortium of Visible Laser Diode Applications

\section{Co-located conferences}

Optics and Photonics International Congress (OPIC) 2021 is a FOUR day event, including ELEVEN cutting edge conferences. OPIC 2021 provides access to the very latest products, research and initiatives in the optics and photonics sector. It also offers you the opportunity for the interaction with those driving the future of optics and photonics technology. And Optics and Photonics International Exhibition (OPIE) 2021, an exciting trade and technology exhibition featuring leading players from across the globe, will be held at the great hall next to the conferences place. That may bring you good opportunities to touch the state of the art products and technologies in the sector. By registering for LDC 2021, you can participate in all international conferences.

\section{$\underline{\text { Scope }}$}

LDC 2021 covers the laser display / lighting technology in the following major topical fields;

\section{A. Light Sources and Components}

Visible lasers, LED, solid-state light sources, phosphor, wavelength conversion materials, for displays and automotive applications. Optoelectronic components, such as spatial light modulators, high-speed scanners, MEMS, projection components, display drivers and interfaces are also included.

\section{B. Imaging / Lighting}


Display, projector, lighting, 3D imaging, and holography systems and technologies. Evaluation and reduction technologies of speckle and color speckle. Color management for laser-based display and lighting. Laser safety and standardization are also included.

\section{Smart Systems}

Display, projector, lighting, 3D imaging, holography, and IoT systems for smart society, which are integrated with other functional devices or technologies, such as AI analysis / control, signal processing, various sensors (e.g., cameras, TOF and LiDAR), human interfaces, wired/wireless microwave/millimeter wave/optical communication devices or interfaces, or wired/wireless optical power supplies. Design, algorithm, or components particularly applied for the above smart systems are also included.

\section{AR, MR, VR, ... XR Technologies}

AR (Augmented Reality), MR (Mixed Reality), and VR (Virtual Reality). These concepts are collectively called XR (Cross Reality). XR technologies opening a new market and having a great impact on life style. New platforms using XR technologies (relevant hardware, software technologies and their applications).

\section{E. Laser Applications for Automotive}

Lighting applications include headlights, rear lights, as well as other functional lighting devices for use in adverse weather conditions. Applications involving both lighting and display include road surface projectors. Sensing applications of particular attention are those for use in autonomous vehicles, such as ToF based LiDAR, as well as FMCW LiDAR. Light-based vehicle-to-vehicle communication will also be featured in this session.

\section{F. Novel and Emerging Technologies}

Novel and emerging technologies and applications of laser lighting and display. Components and optics. Entertainment, education, medical, social applications and others.

\section{Committee}

CONFERENCE CHAIR

\section{Co-Chairs}

Kazuo Kuroda (Utsunomiya Univ.)

Hiroshi Murata (Mie Univ.) 
Fergal Shevlin (DYOPTYKA)

STEERING COMMITTEE

Co-Chairs

Kazuhisa Yamamoto (Osaka Univ.)

Norihiro Ohse (SONY)

\section{Members}

Makio Kurashige (Dai Nippon Printing)

Atsushi Motogaito (Mie Univ.)

Keizo Ochi (Osaka Univ.)

Kayo Ogawa (Japan Women's Univ.)

Shouichi Ozawa (Techno Management Research)

Fumio Narusawa (HIOKI E.E. Corp.)

Shinji Saito (Toshiba Corp.)

Ichiro Sato (Bosch Corp.)

Tsutomu Shimura (Univ. of Tokyo)

Tsuyoshi Suzudo (Ricoh Industrial Solutions)

Takunori Taira (National Inst. of Natural Science)

Masashi Wada (Gooch \& Housego Japan)

\section{PROGRAM COMMITTEE}

\section{Co-Chairs}

Sunao Kurimura (National Inst. for Materials Science)

Tetsuya Yagi (Nichia Corp.)

Shining Zhu (Nanjing Univ.)

\section{Vice co-chairs}

Tatsushi Hamaguchi (SONY)

Muneharu Kuwata (Mitsubishi Electric Corp.)

Hirotsugu Yamamoto (Utsunomiya Univ.)

\section{Members}

Frank Fischer (BST)

Abdelmalek Hanafi (BMW)

Hidekazu Hatanaka (Ushio)

Ray-Hua Horng (National Chiao Tung Univ.) 
Masafumi Ide (Lamda Works)

Takaaki Ishigure (Keio Univ.)

Masato Ishino (Osaka Univ.)

Shunji Kamijima (Epson)

Young-Joo Kim (Yonsei Univ.)

Junichi Kinoshita (Osaka Univ.)

Takuya Kushimoto (Kyoto Univ.)

Masaru Kuramoto (Stanley)

Hisashi Masuda (Oxide)

Osamu Matoba (Kobe Univ.)

Hiroyuki Matsumoto (Iwasaki Electric)

Tomoyuki Miyamoto (Tokyo Inst. Tech.)

Satoshi Ouchi (Hitachi)

Lung-Han Peng (National Taiwan Univ.)

Y-Q Qin (Nanjing Univ.)

Masayuki Takayama (Honda)

\section{ADVISARY MEMBERS}

Ray-Hua Horng (National Chung Hsing Univ.)

Yasuhiro Koike (Keio Univ.)

Shigeo Kubota (Oxide Corp.)

Takashige Omatsu (Chiba Univ.)

Brian Schowengerdt (Univ. of Washington)

Hiroaki Sugiura (Mitsubishi Electric Corp.)

Toshiaki Suhara (Osaka Univ.)

Andreas Tünnermann (Fraunhofer Institute, IOF) 
Mon. Apr 19, 2021

LDC

LDC2021 | Oral Presentation

Opening Remarks

1:00 PM - 1:10 PM LDC (Online)

[LDC-Opening] Opening Remarks

1:00 PM - 1:10 PM

LDC2021 | Oral Presentation

Keynote Session

1:10 PM - 2:40 PM LDC (Online)

[LDC-1-01 (Invited)] Laser Light Sources for Specialty and Automotive Lighting Applications OPaul Rudy ${ }^{1}$ (1.KYOCERA SLD Laser)

1:10 PM - 1:55 PM

[LDC-1-02 (Invited)] Laser Technologies in Novel Medical Imaging

OAlbert P Heberle ${ }^{1} \quad$ (1.Openwater)

1:55 PM - 2:40 PM

LDC2021 | Oral Presentation

AR, MR, VR, ... XR technologies 1 - Aerial \&Aquatic Display -

2:55 PM - 4:10 PM LDC (Online)

[LDC-2-01 (Invited)] 3D Interface by Parity Mirror and Its Applications

OYuki Maeda ${ }^{1}$ (1.Parity Innovations Co., Ltd.)

2:55 PM - 3:25 PM

[LDC-2-02] Subjective Super-Resolution Display on Aerial LED Signage Formed with Aerial Imaging by Retro-Reflection

OKojiro Matsushita ${ }^{1}$, Akinori Tsuji ${ }^{2}$, Toyotaro Tokimoto $^{3}$, Hirotsugu Yamamoto ${ }^{1,4}$ (1.Utsunomiya University, 2.Tokushima University, 3.XAiX, LLC, 4.JST ACCEL)

3:25 PM - 3:40 PM

[LDC-2-03] Multiple Aquatic Image Formation with Faced Mirror Structure and Polarized AIRR

OKazunari Chiba ${ }^{1}$, Masaki Yasugi ${ }^{1,2}$, Hirotsugu Yamamoto $^{1,2}$ (1.Utsunomiya University, 2.JST ACCEL)

3:40 PM - 3:55 PM

[LDC-2-04] Influence of Diverging Angle of the Light Source on the Image Spot Formed in Water by
Use of Retro-reflection

ODaiki Kudo ${ }^{1}$, Kazunari Chiba ${ }^{1}$, Masaki Yasugi ${ }^{1,2}$, Nao Ninomiya ${ }^{1}$, Hirotsugu Yamamoto ${ }^{1,2}$

(1.Utsunomiya university, 2.JST ACCEL)

3:55 PM - 4:10 PM

\section{Tue. Apr 20, 2021}

LDC

LDC2021 | Oral Presentation

$A R, M R, V R, \ldots X R$ technologies 2 - Viewing angle in AR/VR display and devices 10:00 AM - 11:30 AM LDC (Online)

[LDC-3-01 (Invited)] Expanding Field-of-view in Headmounted Displays Considering Characteristics of Vision OWataru Yamada ${ }^{1}$, Hiroyuki Manabe ${ }^{1}$ (1.NTT DOCOMO, INC.) 10:00 AM - 10:30 AM

[LDC-3-02] Analysis on Viewing angle in Aerial Display by Use of an LED Panel Covered with Apertured Retro-Reflector

ODaiki Nishimura ${ }^{1}$, Masaki Yasugi ${ }^{1,2}$, Hirotsugu Yamamoto $^{1,2}$ (1.Utsunomiya University, 2.JST, ACCEL)

10:30 AM - 10:45 AM

[LDC-3-03] Increasing luminance of aerial image formed with AIRR by use of dual transparent spheres OKengo Fujii ${ }^{1}$, Satoshi Maekawa ${ }^{2}$, Hirotsugu Yamamoto $^{1,3}$ (1.Utsunomiya University , 2.Parity Innovations, 3.JST ACCEL)

10:45 AM - 11:00 AM

[LDC-3-04 (Invited)] Compact Full-color Laser Beam Projectors Based on Waveguide-type RGB Multiplexers

OToshio Katsuyama ${ }^{1}$, Akira Nakao ${ }^{1}$, Shoji Yamada ${ }^{1}$, Osamu Kawasaki ${ }^{2}$, Kazuki Iwabata $^{2}$, Koichi Horii ${ }^{2}$, Akira Himeno ${ }^{1,2}$

(1.University of Fukui, 2.SEIREN KST Corp.)

11:00 AM - 11:30 AM

LDC2021 | Oral Presentation

AR, MR, VR, ... XR technologies 3 - xR concepts 3:15 PM - 5:00 PM LDC (Online)

[LDC-4-01 (Invited)] Introduction of xR use case at Toyota 
OKoichi Kayano $^{1} \quad$ (1.Toyota Motor

Corporation)

3:15 PM - 3:45 PM

[LDC-4-02 (Invited)] Introducing KDDI' s activities in XR and Spatial computing towards 5G.

OKatsuhiro Kozuki ${ }^{1}$ (1.KDDI

Corporation)

3:45 PM - 4:15 PM

[LDC-4-03] Deforming Aerial Image by Use of Deflection of Beam Splitter in See-Through AIRR

OKosuke Inoue ${ }^{1}$, Masaki Yasugi ${ }^{1,2}$, Hirotsugu

Yamamoto $^{1,2}$ (1.Utsunomiya University, 2.JST, ACCEL)

4:15 PM - 4:30 PM

[LDC-4-04 (Invited)] Why lasers are key to make smart and AR glasses the next big thing OLucas Ginzinger $^{1} \quad$ (1.Bosch Sensortec $\mathrm{GmbH}$ )

4:30 PM - 5:00 PM

\section{Wed. Apr 21, 2021}

LDC

LDC2021 | Oral Presentation

Imaging / Lighting

10:00 AM - 11:45 AM LDC (Online)

[LDC-5-01 (Invited)] Laser TV progress with visible laser improvement

OXianrong Liu ${ }^{1}$ (1.Hisense)

10:00 AM - 10:30 AM

[LDC-5-02 (Invited)] Large-scale full-parallax full-color computer-generated holograms reconstructed by laser/LED lighting

OKyoji Matsushima ${ }^{1} \quad$ (1.Kansai

University)

10:30 AM - 11:00 AM

[LDC-5-03] Improvement of Color Rendering Index of BGYR Laser illuminats

OYoshio Manabe ${ }^{1}$, Masato Ishino ${ }^{1}$, Hiroshi Fuji ${ }^{1}$, Akira Takamori ${ }^{1}$, Junichi Kinoshita ${ }^{1}$, Kana Fujioka ${ }^{1}$, Kazuhisa Yamamoto ${ }^{1}$ (1.Institute of Laser Engineering, Osaka University)

11:00 AM - 11:15 AM

[LDC-5-04] Color Shift Behavior at Image Pattern Edges of Raster-scan RGB Mobile Laser Projectors

OJunichi Kinoshita ${ }^{1}$, Akira Takamori ${ }^{1}$, Kazuhisa
Yamamoto $^{1}$, Kazuo Kuroda ${ }^{2}$, Koji Suzuki ${ }^{3} \quad$ (1.Osaka Universitry, 2.Utsunomiya University, 3.OXIDE

Corporation)

11:15 AM - 11:30 AM

[LDC-5-05] Ultra high resolution and VAC-free N3D technology and its applications

OChin-Yung Hsieh ${ }^{1}$, Hao-Yu Liu ${ }^{1}$, Ruey-Jer Weng ${ }^{1}$, Wei-Yi Lu ${ }^{1}$, Naoki Sumi ${ }^{2}$ (1.Innolux Corporation,

2.Innolux Japan K.K.)

11:30 AM - 11:45 AM

LDC2021 | Oral Presentation

Light sources and components

1:00 PM - 2:30 PM LDC (Online)

[LDC-6-01 (Invited)] Nanowire LEDs with Ultrastable

Emission Characteristics and

Monolithically Integrated Multicolour

Emission

OZetian $\mathrm{Mi}^{1}$, Xianhe Liu ${ }^{1}$, Yi Sun ${ }^{1}$,

Yakshita Malhotra ${ }^{1}$, Yuanpeng $\mathrm{Wu}^{1}$

(1.University of Michigan)

1:00 PM - 1:30 PM

[LDC-6-02 (Invited)] Emission Characteristics of Random Lasers and Their Control

OTakashi Okamoto ${ }^{1}$ (1.Kyushu Institute

of Technology)

1:30 PM - 2:00 PM

[LDC-6-03 (Invited)] Purcell-Effect-Enhanced Red

Emission from Eu lons in $\mathrm{GaN}$

Cavities

OYasufumi FUJIWARA ${ }^{1}$, Dolf

TIMMERMAN ${ }^{1}$, Shuhei ICHIKAWA ${ }^{1}$, Jun

TATEBAYASHI $^{1}$ (1.Osaka University)

2:00 PM - 2:30 PM

LDC2021 | Oral Presentation

Laser technology for automotive applications 1 2:45 PM - 3:45 PM LDC (Online)

[LDC-7-01 (Invited)] Laser Crystal Phosphor Automobile Headlight Integrated with Beam Control and LiDAR OKenneth $\mathrm{Li}^{1}$, Y. P. Chang ${ }^{2}$

(1.Optonomous Technologies Inc., 2.Taiwan Color Optics, Inc.) 2:45 PM - 3:15 PM

[LDC-7-02] Improvement of Color LiDAR with RGB visible 
single-mode laser diodes

OMasato Ishino ${ }^{1}$, Tomoyuki Ohashi ${ }^{1}$, Hiroshi Fuji ${ }^{1}$,

Kana Fujioka ${ }^{1}$, Kazuhisa Yamamoto ${ }^{1}$ (1.Osaka

University)

3:15 PM - 3:30 PM

[LDC-7-03] Thermal property of AIN-Ce:YAG composite ceramics phosphor for laser lighting OTakuya Sawada ${ }^{1}$, Hiroshi Huji ${ }^{1}$, Kenta Yagasaki ${ }^{1}$, Hisashi Minemoto ${ }^{1}$, Yukio Manabe ${ }^{1}$, Kana Fujioka ${ }^{1}$, Kazuhisa Yamamoto ${ }^{1}$ (1.Institute of Laser Engineering, Osaka University)

3:30 PM - 3:45 PM

LDC2021 | Oral Presentation

Laser technology for automotive applications 2 4:00 PM - 5:00 PM LDC (Online)

[LDC-8-01(Invited)] GaN-based Vertical-Cavity SurfaceEmitting Lasers with Lattice-Matched AllnN/GaN DBRs

OTetsuya Takeuchi ${ }^{1}$, Satoshi Kamiyama ${ }^{1}$, Motoaki Iwaya ${ }^{1}$, Isamu Akasaki ${ }^{1,2}$ (1.Meijo University, 2.Nagoya University) 4:00 PM - 4:30 PM

[LDC-8-02] Freeform Lens Design for High-Efficient LED Low-Beam Headlamp Lens

OZhengbo Zhu ${ }^{1}$, Shili Wei ${ }^{1}$, Wenyi $\mathrm{Li}^{1}$, Donglin $\mathrm{Ma}^{1}$

(1.Huazhong University of Science and

Technology)

4:30 PM - 4:45 PM

[LDC-8-03] High Power Laser Phosphor Light Source using Tilted Rotating Mirror

OKenneth $\mathrm{Li}^{1}$, Y. P. Chang ${ }^{2}$, Lion Wang ${ }^{2}$, Andy Chen $^{2}$, Stark Tsai ${ }^{2}$ (1.Optonomous Technologies Inc., 2.Taiwan Color Optics, Inc.)

4:45 PM - 5:00 PM

\section{Thu. Apr 22, 2021}

\section{LDC}

LDC2021 | Oral Presentation

Smart systems

1:00 PM - 2:30 PM LDC (Online)

[LDC-9-01 (Invited)] Geo-Marine Science using Visible Light

OHiroshi Yoshida ${ }^{1}$, Shojiro Ishibashi ${ }^{1}$

(1.JAMSTEC)
1:00 PM - 1:30 PM

[LDC-9-02] Imperceptible Projected Marker Codes with Application to Calibration-Free Projection Mapping

OShingo Kagami ${ }^{1}$ (1.Tohoku University)

1:30 PM - 2:00 PM

[LDC-9-03] Scanning RGB Laser Beam Detection for Smart Laser Display System

OTakeshi Ebara ${ }^{1}$, Hiroshi Murata ${ }^{1,2}$, Masato Ishino ${ }^{2}$, Junichi Kinoshita ${ }^{2}$, Kazuhisa Yamamoto ${ }^{2}$ (1.Mie

University, 2.Institute of Laser Engineering, Osaka

University)

2:00 PM - 2:15 PM

[LDC-9-04] Extraction Method of Typical Traffic Lines of Pedestrians

OAkinobu Watanabe $^{1}$ (1.Hitachi, Ltd.)

2:15 PM - 2:30 PM

LDC2021 | Oral Presentation

Novel and Emerging Technologies

2:45 PM - 3:15 PM LDC (Online)

[LDC-10-01] Design an Extreme Ultraviolet Illumination System with High Uniformity

OYanqiu Li ${ }^{1}$, Qian Hao ${ }^{1}$, Ke Liu ${ }^{1}$, Xu Yan ${ }^{1}$

(1.Beijing Institute of Technology)

2:45 PM - 3:00 PM

[LDC-10-02] Colorization of Arc3D using Projector Lighting

Olkuya Saji ${ }^{1}$, Masafumi Nakata ${ }^{2}$, Yasuhiro

Kashihara ${ }^{2}$, Atsushi Hayashi ${ }^{2}$, Hirotsugu

Yamamoto $^{1,3}$ (1.Utsunomiya University, 2.NSC

Co., Ltd., 3.JST, ACCEL)

3:00 PM - 3:15 PM

LDC2021 | Oral Presentation

Closing Remarks

3:15 PM - 3:25 PM LDC (Online)

[LDC-Closing] Closing Remarks

3:15 PM - 3:25 PM 
LDC2021 | Oral Presentation

\title{
Opening Remarks
}

Mon. Apr 19, 2021 1:00 PM - 1:10 PM LDC (Online)

Click to the Online Room

\section{[LDC-Opening] Opening Remarks}

\author{
1:00 PM - 1:10 PM
}

Proc. of SPIE Vol. 11851 1185101-10

Downloaded From: https://www.spiedigitallibrary.org/conference-proceedings-of-spie on 26 Apr 2023 Terms of Use: https://www.spiedigitallibrary.org/terms-of-use 
LDC2021 | Oral Presentation

\section{Keynote Session}

Mon. Apr 19, 2021 1:10 PM - 2:40 PM LDC (Online)

Click to the Online Room

\section{[LDC-1-01 (Invited)] Laser Light Sources for Specialty and Automotive Lighting} Applications

OPaul Rudy ${ }^{1}$ (1.KYOCERA SLD Laser)

1:10 PM - 1:55 PM

[LDC-1-02 (Invited)] Laser Technologies in Novel Medical Imaging

OAlbert $\mathrm{P}$ Heberle ${ }^{1} \quad$ (1.Openwater)

1:55 PM - 2:40 PM 
LDC2021 | Oral Presentation

Keynote Session

Mon. Apr 19, 2021 1:10 PM - 2:40 PM LDC (Online)

\section{[LDC-1-01 (Invited)] Laser Light Sources for Specialty and Automotive Lighting Applications \\ OPaul Rudy ${ }^{1}$ (1.KYOCERA SLD Laser)}

We provide a description on the rapidly expanding capabilities of laser light technology, including 1000 lumen sources with luminance above $1000 \mathrm{~cd} / \mathrm{mm} 2$, more than $10 x$ that of LEDs, from surface mount device components. 


\title{
Laser Light Sources for Specialty and Automotive Lighting Applications
}

\author{
Paul Rudy \\ KYOCERA SLD Laser, Inc., 485 Pine Ave, Goleta, CA 93117, (408) 921-6948, PRudy@kyocera-sldlaser.com
}

\begin{abstract}
We provide a description on the rapidly expanding capabilities of laser light technology, including 1000 lumen sources with luminance above $1000 \mathrm{~cd} / \mathrm{mm} 2$, more than $10 x$ that of LEDs, from surface mount device components, along with compact lightweight spotlight arrays exceeding 50,000 lumens with a $10 \mathrm{~km}$ beam distance.
\end{abstract}

We provide a description on the rapidly expanding capabilities of laser light technology, including 1000 lumen sources with luminance above $1000 \mathrm{~cd} / \mathrm{mm} 2$, more than 10x that of LEDs, from surface mount device components, along with compact lightweight spotlight arrays exceeding 50,000 lumens with a $10 \mathrm{~km}$ beam distance. These sources are $6000 \mathrm{~K}$ and $70 \mathrm{CRI}$, and are optimized for outdoor illumination applications. Precision beam shaping with static diffusers and dynamic control demonstrations are also described.

We also report on the world's first dual emission white light and infrared (IR) source for automotive and consumer lighting, night vision illumination, precision long-range sensing beyond 250 meters, and high-speed LiFi communications faster than 20 gigabit per second. The new dual White/IR sources deliver high brightness, safe incoherent white light of 500 lumens with near infrared emission up to 1 watt average power and 100 watt peak power that can emit either together simultaneously or independently, depending on the needs of the application. The White/IR source technology has been configured into compact spotlight modules and also delivered via fiber optics in transport fibers as well as emissive fiber arrangements. Until now, high speed dual emission white/IR sources have not been possible because LEDs and legacy lampbased light sources are unable to deliver high brightness dual wavelength emission from the same point source, and they are incapable of being modulated at the high speeds required for accurate sensing and fast data rates.

When configured with sensors and compact optics, the new dual emission source delivers high brightness white light with $1 \mathrm{~km}$ beam distance, while independently producing IR illumination from the same emission spot to achieve ranging to more than 250 meters with $1 \%$ accuracy. Moreover, these sources generate LiDAR video imagery and data when integrated with sensor chips, to enable next generation $3 \mathrm{D}$ imaging headlights. White/IR dual emission sources are critical for automotive lighting, advanced driver assistance systems (ADAS), and autonomous vehicle LiDAR 3D sensing, as well as mobility applications including avionics, drones, railway, and marine. Additionally, dual emission sources are required for consumer and professional product portable lighting products, night vision illuminators, and rangefinders for recreation and outdoor, search \& rescue, and security applications.

Utilizing the new 1000 lumen source, high efficiency fiber delivery of the white light is demonstrated with transport and emissive fiber illumination up to $10,000 \mathrm{~cd} / \mathrm{m} 2$ for ultra-bright lighting for vehicle exterior grills, logos, and interiors. With 10 times the brightness of LED solutions, these sources produce brilliant and efficient illumination from thin, low cost, plug-and-play fiber optics and a modular light source, with simplified maintenance and enhanced thermal management.

Utilizing the White/IR dual-emission source, we present spotlight and fiber modules that deliver more than 20 gigabit per second data rates for $\mathrm{LiFi}$ communication applications. Additionally, we report on a compact $\mathrm{LiFi}$ development module with 1 gigabit per second data rate for emerging optical communication applications for a myriad of mobility use cases, as well as future smart cities such as intelligent streetlights, and smart buildings such as healthcare facilities and factories. By delivering both white and IR from the same source and fusing together lighting, sensing, and communication functionalities, the LiFi modules provide promise for future intelligent illumination systems with a unique combination of precision white lighting, accurate sensing and ultra-high speed communication with unconstrained optical bandwidth, as well as secure and efficient data transmission without RF interference of the incumbent WiFi technology. 
LDC2021 | Oral Presentation

Keynote Session

Mon. Apr 19, 2021 1:10 PM - 2:40 PM LDC (Online)

\section{[LDC-1-02 (Invited)] Laser Technologies in Novel Medical Imaging}

OAlbert P Heberle ${ }^{1}$ (1.Openwater)

We are devising a new generation of portable medical imaging technologies for improved access to medical diagnoses and treatments. Our breakthrough systems use opto-electronics and holography together with novel lasers to enable low cost equipment that rivals the resolution and image quality of multi-million dollar medical imaging scanners. 


\title{
Laser technologies in novel medical imaging
}

\author{
Albert P. Heberle \\ Openwater, 733 Front St., Suite C1A, San Francisco, CA 94111, albert@openwater.cc
}

\begin{abstract}
We are devising a new generation of portable medical imaging technologies for improved access to medical diagnoses and treatments. Our breakthrough systems use opto-electronics and holography together with novel lasers to enable low cost equipment that rivals the resolution and image quality of multi-million dollar medical imaging scanners.
\end{abstract}

Proc. of SPIE Vol. 11851 1185101-15 
LDC2021 | Oral Presentation

\section{AR, MR, VR, ... XR technologies 1 - Aerial \&Aquatic Display -}

Mon. Apr 19, 2021 2:55 PM - 4:10 PM LDC (Online)

Click to the Online Room

[LDC-2-01 (Invited)] 3D Interface by Parity Mirror and Its Applications

OYuki Maeda ${ }^{1}$ (1.Parity Innovations Co., Ltd.)

2:55 PM - 3:25 PM

[LDC-2-02]

Subjective Super-Resolution Display on Aerial LED Signage

Formed with Aerial Imaging by Retro-Reflection

OKojiro Matsushita ${ }^{1}$, Akinori Tsuji ${ }^{2}$, Toyotaro Tokimoto ${ }^{3}$, Hirotsugu Yamamoto ${ }^{1,4}$

(1.Utsunomiya University, 2.Tokushima University, 3.XAiX, LLC, 4.JST ACCEL)

[LDC-2-03]

3:25 PM - 3:40 PM

Multiple Aquatic Image Formation with Faced Mirror Structure and Polarized AIRR

OKazunari Chiba ${ }^{1}$, Masaki Yasugi ${ }^{1,2}$, Hirotsugu Yamamoto ${ }^{1,2}$ (1.Utsunomiya

University, 2.JST ACCEL)

3:40 PM - 3:55 PM

[LDC-2-04] Influence of Diverging Angle of the Light Source on the Image Spot Formed in Water by Use of Retro-reflection

ODaiki Kudo ${ }^{1}$, Kazunari Chiba ${ }^{1}$, Masaki Yasugi ${ }^{1,2}$, Nao Ninomiya ${ }^{1}$, Hirotsugu

Yamamoto $^{1,2}$ (1.Utsunomiya university, 2.JST ACCEL)

3:55 PM - 4:10 PM 
LDC2021 | Oral Presentation

$\mathrm{AR}, \mathrm{MR}, \mathrm{VR}, \ldots \mathrm{XR}$ technologies 1 - Aerial \&Aquatic Display -

Mon. Apr 19, 2021 2:55 PM - 4:10 PM LDC (Online)

\section{[LDC-2-01 (Invited)] 3D Interface by Parity Mirror and Its Applications}

OYuki Maeda ${ }^{1}$ (1.Parity Innovations Co., Ltd.)

An optical imaging device for air floating image display and its applications are introduced in this paper. An observer can see the air floating image by the naked eye and manipulate it by touching the air floating image using non-contact sensor. 


\title{
3D Interface by Parity Mirror and Its Applications
}

\author{
Yuki Maeda ${ }^{1}$ \\ ${ }^{1}$ Parity Innovations Co. Ltd., 1-5-1, Aramoto-kita, Higashiosaka-shi, Osaka, JAPAN, +81-6-6753-8244, maeda@piq.co.jp
}

\begin{abstract}
An optical imaging device for air floating image display and its applications are introduced in this paper. An observer can see the air floating image by the naked eye and manipulate it by touching the air floating image using non-contact sensor.
\end{abstract}

\section{Introduction}

An air floating image display have appeared frequently in science fiction movies, and their realization and practical application have been expected. In recent years, technological development of air floating image displays using imaging optics has progressed [1-3], and one of them is based on retro-reflection by micromirror array. Parity Innovations Co. Ltd. has developed Parity mirror, that is an optical imaging device based on a dihedral corner reflector array. Furthermore, a non-contact user interface, such as an air floating switch and an air floating touch display, has been developed as an application product using the Parity mirror. This technology is attracting a lot of attention because it can prevent contact infections in infectious diseases such as COVID-19, that the virus can survive for a long time on the surface of an object [4]. In this paper, basic principle of air floating imaging and applications using the Parity mirror are introduced.

\section{Parity mirror}

\subsection{Imaging}

Parity mirror can be made from transparent resin by thermal nanoimprint with a fine metallic stamper. Figure 1 shows a schematic diagram of the Parity mirror and the ray paths. The Parity mirror consists of many pillars whose sidewall works as a roof mirror, that is, the adjacent two sidewalls are orthogonal and vertical planar reflecting surfaces. Note that there are draft angles on the opposite side of the adjacent two walls for moldreleasing in process of thermal nanoimprint; these draft angle surfaces cannot form an air floating image. Typically, the size of the pillar is approximately $200 \mu \mathrm{m}$ square, and the depth of the pillar is approximately $300 \mu \mathrm{m}$. Some incident rays are reflected twice in the pillar by total internal reflection and invert the horizontal component, as shown in Fig. 1 (a). On the other hand, vertical component of the incident rays does not invert and path through the Parity mirror, as shown in Fig. 1 (b). The optical path of the twice-reflected ray is planesymmetric with respect to the Parity mirror. Accordingly, an air floating image (real image) of an object is formed at the plane-symmetric point. An observer can see the floating image when the image is viewed obliquely from above. No reflection (direct passing), once reflection and multiple (three or more times) reflection are not formed an air floating image but observed as stray light.

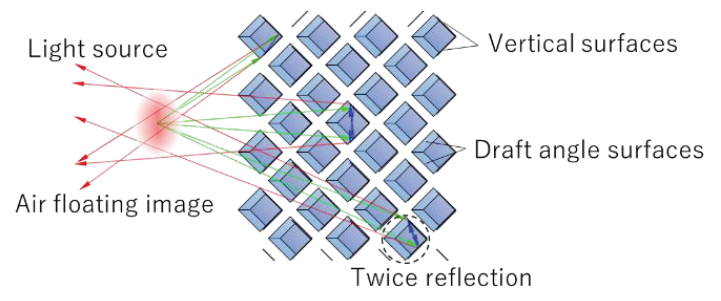

(a)

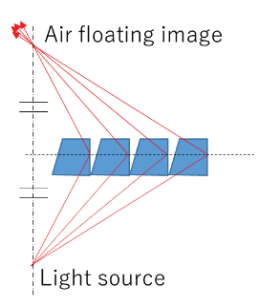

(b)

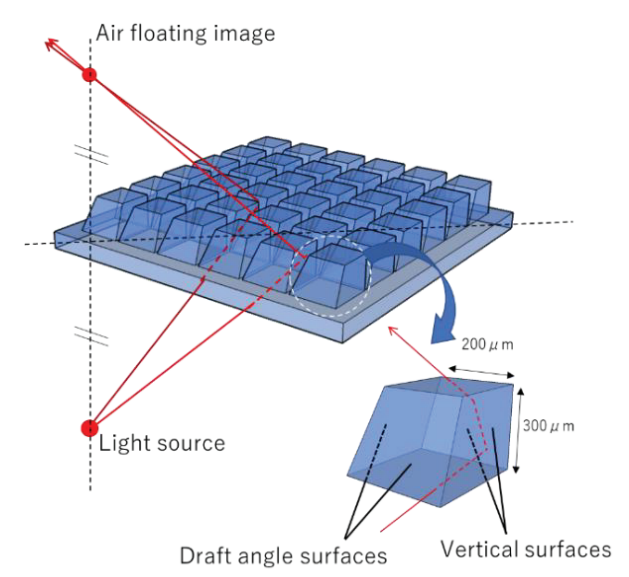

(c)

Fig. 1. Schematic illustration of the transparent material type DCRA and rays. (a) is top view. (b) is side view. (c) is overhead view with enlarged view of unit optical system.

\subsection{Imaging}

The Parity mirror can be manufactured by thermal nanoimprint using a metal stamper made by nano processing machine. Current maximum size is $300 \mathrm{~mm}$ square. The material is acrylic resin. As post-processing, shading is required to reduce stray light and improve contrast. Figure 2 shows a sample of Parity mirror. An 
effective incident angle for twice reflection is \pm 15 degrees horizontally from the center and from 20 degrees to 50 degrees vertically. Imaging efficiency is about $13 \%$, so it is desirable to use a high brightness light source.

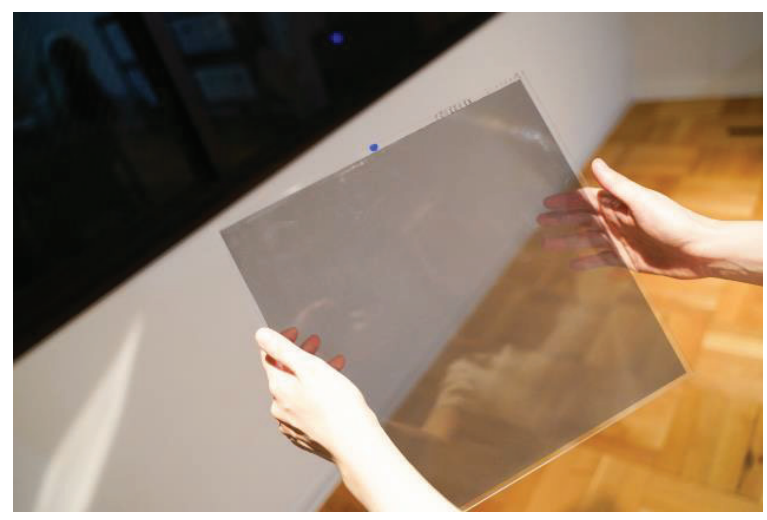

Fig. 2. Parity mirror with shading processing.

\section{Applications}

A non-contact user interface can be developed using the Parity mirror with a non-contact sensor, such as an infrared sensor and a camera system. Figure 3 shows the non-contact switch devices series named "AiR Switch". An infrared Time-of-Flight sensor was used as the noncontact sensor. An LED backlight and printed film were used as the light source. For example, by connecting AiR Switch to a desk light, the light can be turned on and off in a non-contact manner. In addition, by incorporating it into a building or device in advance, it is possible to operate the number of floors in elevator or a toilet flush button without physical contact. It is considered that replacing the operation device in public space with AiR Switch is effective to prevent contact infections in infectious diseases such as COVID-19.

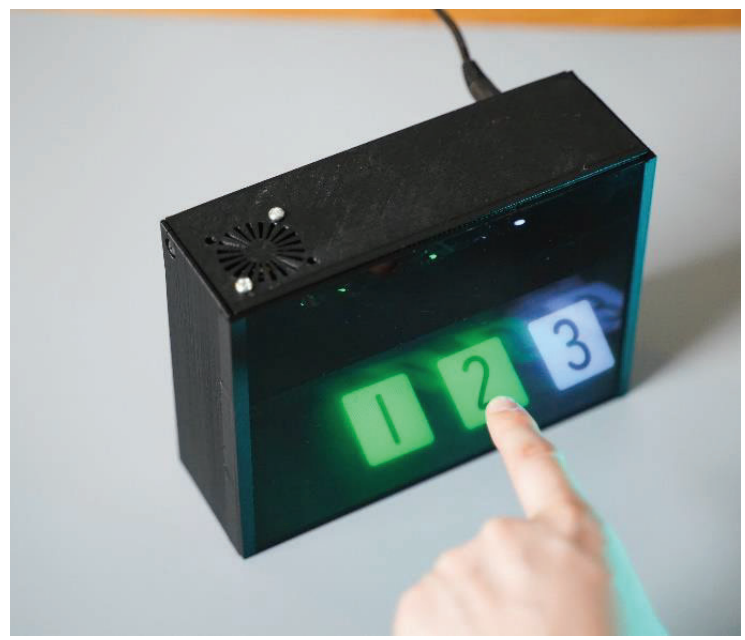

Fig. 3. AiR Switch.
Another example is named AIRIA (AIR InteRactive display) that enables more complicated non-contact operations, As shown in Fig. 4.

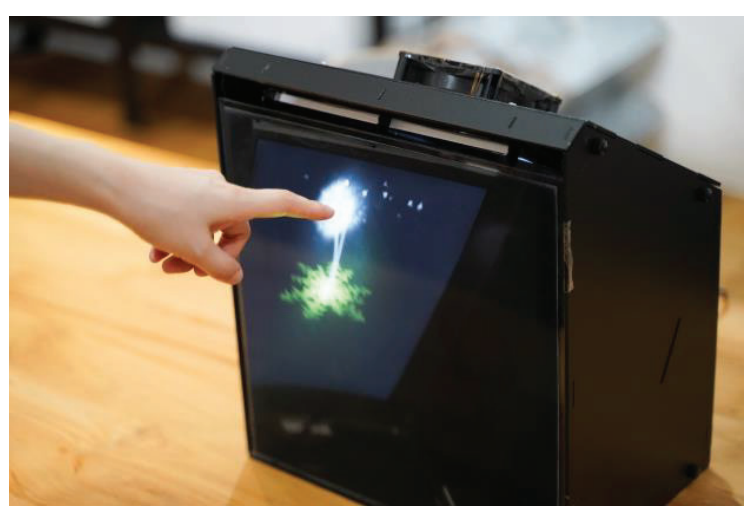

Fig. 4. AIRIA.

\section{Conclusion}

In this paper, basic principles and applications of the Parity mirror were introduced. The Parity mirror forms a distortion-free floating image that can be seen fixed positions in the air regardless of the direction and distance of viewing. AiR Switch and AIRIA were introduced as examples of non-contact user interfaces. The Parity mirror can be manufactured with a transparent material, such as acrylic resin, by thermal nanoimprint. For market expansion, we are working to reduce manufacturing costs and improve optical performance such as light efficiency. Moreover, upsizing and expansion of observation angle are important future works.

\section{References}

[1] S. Maekawa, K. Nitta, and O. Matoba, "Transmissive Optical Imaging Device with Micromirror Array," Proc. SPIE 6392, 63920E (2006).

[2] D. Miyazaki, N. Hirano, Y. Maeda, S. Yamamoto, T. Mukai, and S. Maekawa, "Floating volumetric image formation using a dihedral corner reflector array device," Appl. Opt. 52(1), A281-A289 (2013).

[3] H. Yamamoto and S. Suyama, "Aerial 3D LED display by use of retroreflective sheeting," Proc. SPIE 8648, 86480Q (2013).

[4] N. van Doremalen, T. Bushmaker, D. H. Morris, M. G. Holbrook, A. Gamble, B. N. Williamson, A. Tamin, J. L. Harcourt, N. J. Thornburg, S. I. Gerber, J. O. Lloyd-Smith, E. de Wit, and V. J. Munster, "Aerosol and Surface Stability of SARS-CoV-2 as Compared with SARS-CoV1," N Engl J Med. 382(16), 1564-1567 (2020). 
LDC2021 | Oral Presentation

$A R, M R, V R, \ldots X R$ technologies 1 - Aerial \&Aquatic Display -

Mon. Apr 19, 2021 2:55 PM - 4:10 PM LDC (Online)

\section{[LDC-2-02] Subjective Super-Resolution Display on Aerial LED Signage Formed with Aerial Imaging by Retro-Reflection}

OKojiro Matsushita ${ }^{1}$, Akinori Tsuji ${ }^{2}$, Toyotaro Tokimoto ${ }^{3}$, Hirotsugu Yamamoto ${ }^{1,4}$ (1.Utsunomiya University, 2.Tokushima University, 3.XAiX, LLC, 4.JST ACCEL)

We have realized a novel aerial display that features higher resolution than the light-source LED. We have realized subjective super-resolution display, which makes viewers perceive finer resolution than the actual number of pixels, on aerial LED signage by use of our designed high-speed LED display circuit and aerial imaging optics. 


\title{
Subjective Super-Resolution Display on Aerial LED Signage Formed with Aerial Imaging by Retro-Reflection
}

\author{
Kojiro Matsushita ${ }^{1}$, Akinori Tsuji ${ }^{2}$, Toyotaro Tokimoto ${ }^{3}$, Hirotsugu Yamamoto ${ }^{1,4}$ \\ ${ }^{1}$ Utsunomiya University, Yoto 7-1-2, Utsunomiya City, Tochigi, 321-0904, Japan, \\ E-mail: hirotsugu@yamamotolab.science \\ ${ }^{2}$ Tokushima University, Minamijosanjima-cho 2-1, Tokushima City, Tokushima, 770-8501, Japan, \\ ${ }^{3}$ XAiX, LLC, Minatomirai 5-3-1-1904, Nishi-ku, Yokohama City, Kanagawa, 220-0012, Japan, \\ ${ }^{4}$ JST ACCEL, Yoto 7-1-2, Utsunomiya City, Tochigi, 321-0904, Japan.
}

\begin{abstract}
We have realized a novel aerial display that features higher resolution than the light-source LED. We have realized subjective super-resolution display, which makes viewers perceive finer resolution than the actual number of pixels, on aerial LED signage by use of our designed high-speed LED display circuit and aerial imaging optics.
\end{abstract}

\section{Introduction}

Subjective super-resolution display is a novel display method to make viewers perceive finer resolution than the physical resolution of the source display [1]. Subjective super-resolution display shows the image data adjacent to a low-density LED element alternate at a high speed so that it looks like an illusion of higher resolution than the resolution of an actual LED. We have developed a variable frame rate LED display by use of FPGA (field programmable gate array) for subjective super-resolution effect by showing 4-neighouring subpixel information [2]. Furthermore, we have increased the number of virtual resolutions so that $3 \times 3$ dots in the original image are assigned to 1 pixel in an LED panel [3]. Adjacent $3 \times 3$ dots are divided so that they overlap 3 dots and shown on an LED panel with FPGA circuit.

This paper firstly reports the implementation of the subjective super-resolution display on an aerial LED signage. In this work, we employ aerial imaging by retroreflection (AIRR) [4] and an LED panel with our designed high-speed driving circuit for subjective super-resolution display. Although AIRR features a blurred imaging that interpolate black regions between LED lamps [4], the perceived resolution with this normal aerial LED signage is the same as the resolution of the light-source LED panel. By introducing subjective super-resolution display method, we aim to show finer resolution on an aerial LED signage than the original LED panel. In Section 2, we describe the principle of the subjective super-resolution. Aerial imaging optics is explained in Section 3. In Section 4 , we report the experimental setups and results.

\section{Subjective super-resolution display}

Subjective super-resolution display is realized by dividing a high-resolution original image into multiple subframes according to the size of the display and switching at high speed [1-3]. Fig. 1 shows the principle of subjective superresolution.

Subjective super-resolution utilizes fixational eye movement, which is the movement of the eyeball that occurs constantly unconsciously and consists of microsaccade, drift, and tremor [5]. Fig. 2 illustrates the movements of the line of sight due to fixational eye movement. We do not perceive the movement of the eyeball due to fixational eye movement. Because there is a circuit in the brain to correct the flickering caused by fixational eye movement [6]. The observer perceives that the movement due to the switching of the subframes is due to the shaking of his or her own eyeball, and by correcting in the brain, the observer can see more smoothly than the resolution of the actual display.

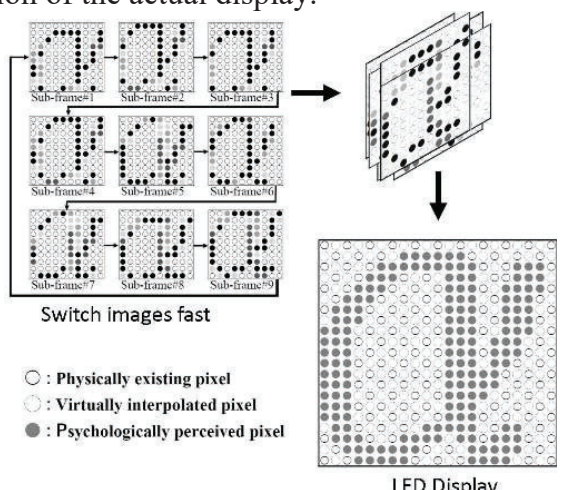

Fig. 1. Principle of subjective super-resolution display.

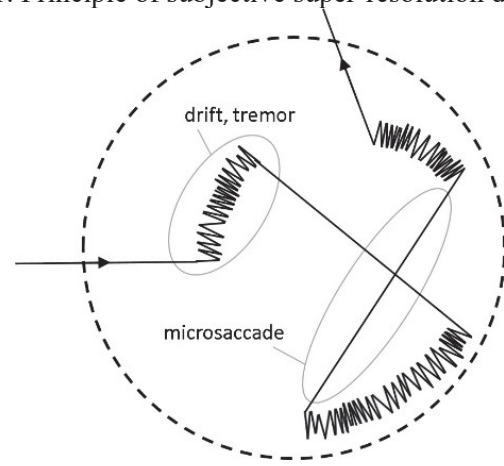

Fig. 2. Fixational eye movement.

\section{AIRR}

AIRR is one of the aerial imaging technologies and consists of a light source, a retro-reflector, and a beam splitter [4]. Fig. 3 shows the principle of AIRR. The light from the light source is reflected by the beam splitter and travels reversely after the retro-reflection. The light transmitted through the beam splitter converges to the 
plane symmetrical position of the light source with respect to the beam splitter.

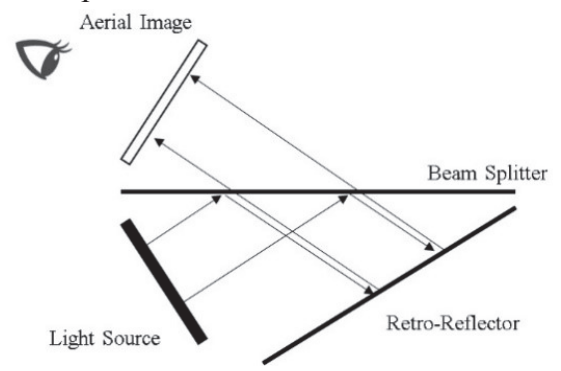

Fig. 3. Principle of AIRR.

\section{Experiment}

We used a binary image in $65 \times 65$ pixels as the original image and divided it into 9 subframes in $32 \times 32$ pixels. 9 subframes are obtained by extracting every other pixel based on each of the upper left 9 pixels of the original image. Fig. 4 shows the correspondence between the original image and the subframes. For the original image of $(2 n+1) \times(2 n+1)$ pixels, $n \times n$ pixels subframe are created. Fig. 5 shows the subframe used in this experiment. Nine slightly different subframes have been created. In other words, information for nine pixels is displayed for one actual pixel. Because the super-resolution effect is known to increase near $64 \mathrm{~Hz}$, the subframe switching frequency was set to $64 \mathrm{~Hz}$ [3]. We investigate the difference between directly viewing the LED panel and viewing the aerial LED signage.
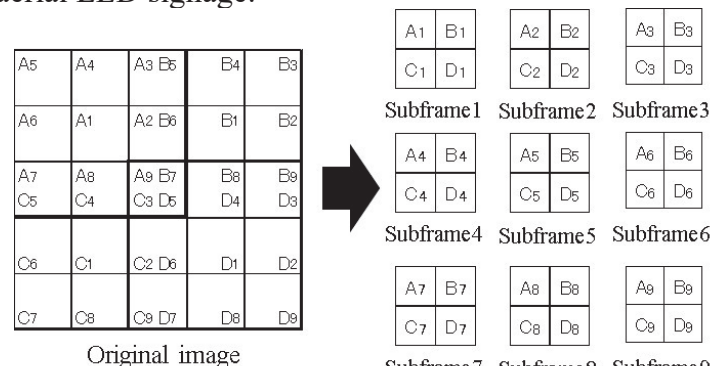

Fig. 4. Original image and subframes.

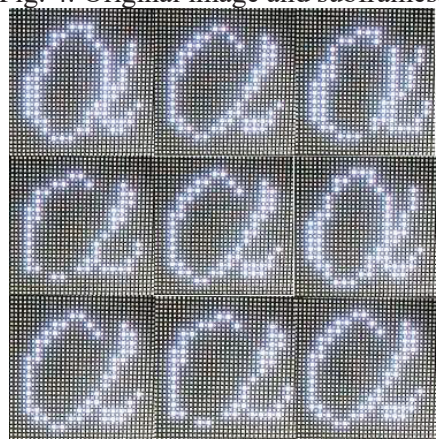

Fig. 5. Nine subframes

The left and the right in Fig. 6 are photographs of the source LED display and the aerial display, respectively. Observation results shows that the perceived resolution was higher in the aerial display. When we looked directly at the LED display in a bright place, the static existence of the LED pixels was noticeable. However, in the aerial image, the existence of the static pixels was invisible and only the bright pixels were visible. This difference makes the super-resolution effects higher.

Fig. 7 shows a comparison image in a dark place. In this case, when we looked directly at the source LED display, we saw the bright pixels. However, the superresolution effects of aerial images were higher than looking directly in the dark. When looked directly, the pixels of the LED are clearly visible, whereas the outlines of the pixels are blurred in the aerial image. It is thought that the blurring of the outline of each pixel facilitates correction in the brain.

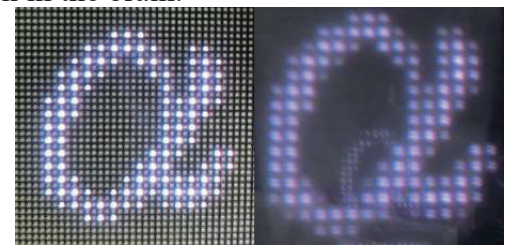

Fig. 6. Super-resolution effects in a bright place.

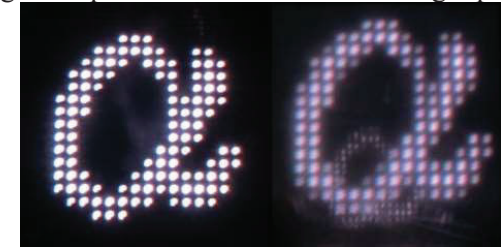

Fig. 7. Super-resolution effects in a dark place.

\section{Conclusion}

In this work, we constructed a system for subjective super-resolution LED display corresponding to 8 colors. We have confirmed by experiments that subjective superresolution is effective for multi-color images. Further studies are needed in order to verify the effectiveness of subjective super-resolution display in full color and superresolution effects in landscape images. A part of this work was supported by JST/ACCEL (grant no. JPMJAC1601) and JSPS KAKENHI (19H04155, 20H05702).

\section{References}

[1] T. Tokimoto, S. Tokimoto, K Fujii, S. Morita, and H. Yamamoto, "Subjective Super-Resolution Model on Coarse High-Speed LED Display in Combination with Pseudo Fixation Eye Movements," IEICE Trans. Electron., vol. E102-C, No. 11, pp. 780-788 (2019).

[2] T. Tokimoto, S. Tokimoto, K Fujii, S. Morita, and H. Yamamoto, "Subjective Super-Resolution Model on Coarse High-Speed LED Display in Combination with Pseudo Fixation Eye Movements," IEICE Trans. Electron., vol. E102-C, No. 11, pp. 780-788 (2019).

[3] K. Matsushita, T. Tokimoto, K. Fujii, H. Yamamoto, "Variable Frame Rate LED Display for Subjective SuperResolution," IMID2019, P02-102, pp. 107-108 (2019).

[4] H. Yamamoto, Y. Tomiyama, and S. Suyama, "Floating aerial LED signage based on aerial imaging by retroreflection (AIRR)," Opt. Express, vol. 22, pp. 2691926924 (2014).

[5] H. Kaneko, "Fixational eye movement," Journal of The Institute of Image Information and Television Engineers, vol. 63, pp. 1538-1539(2010)[in Japanese].

[6] S. Martinez-Conde, S. L. Macknik, and D. H. Hubel, "The role of fixational eye movements in visual perception," Nature Reviews Neuroscience, vol. 5, pp. 229-240 (2004). 
LDC2021 | Oral Presentation

$\mathrm{AR}, \mathrm{MR}, \mathrm{VR}, \ldots \mathrm{XR}$ technologies 1 - Aerial \&Aquatic Display -

Mon. Apr 19, 2021 2:55 PM - 4:10 PM LDC (Online)

\section{[LDC-2-03] Multiple Aquatic Image Formation with Faced Mirror Structure and Polarized AIRR}

OKazunari Chiba ${ }^{1}$, Masaki Yasugi ${ }^{1,2}$, Hirotsugu Yamamoto ${ }^{1,2}$ (1.Utsunomiya University, 2.JST ACCEL)

This paper proposes a new way of 3D display application. Multiple aquatic images were formed using the principle of infinity mirror and Aerial Imaging by Retro-Reflection (AIRR). We have successfully improved the brightness of aquatic images by use of polarization modulation. 


\title{
Multiple Aquatic Image Formation with Faced Mirror Structure and polarized AIRR
}

\author{
Kazunari Chiba ${ }^{1}$, Masaki Yasugi ${ }^{1,2}$, Hirotsugu Yamamoto ${ }^{1,2}$ \\ ${ }^{1}$ Utsunomiya University, Yoto 7-1-2, Utsunomiya City, Tochigi \\ ${ }^{2}$ JST ACCEL, Yoto 7-1-2, Utsunomiya City, Tochigi
}

\begin{abstract}
This paper proposes a new way of $3 D$ display application. Multiple aquatic images were formed using the principle of infinity mirror and Aerial Imaging by Retro-Reflection (AIRR). We have successfully improved the brightness of aquatic images by use of polarization modulation.
\end{abstract}

\section{Introduction}

Currently, various technologies for forming images in the mid-air have been proposed. One of them is aerial imaging by retro-reflection (AIRR) [1], which features a wide viewing angle and a large-size scalability. This paper proposes a new way of $3 \mathrm{D}$ display application. We utilize aerial display technique for underwater aquatic display. In previous research, a method to form multiple aquatic images [2] by using a single LED light source by introducing the optical system of infinity mirror into AIRR. However, only about two rows of aerial images can be seen due to the decrease in brightness.

In this study, we propose a method to improve the brightness of multiple aquatic images by use of polarized AIRR [3].

\section{Principle}

2.1 AIRR (Aerial Imaging by Retro-Reflection) The principle of AIRR is shown in Fig. 1. AIRR consists of a light source, a beam splitter, and a retro-reflector. Light from the light source goes to the beam splitter and splits in transmitted and reflected light. The reflected light reaches the retro-reflector. The retro-reflected light splits again on the beam splitter. The transmitted light forms the aerial image. The aerial image is formed plane-symmetrically of the light source with respect to the beam splitter.

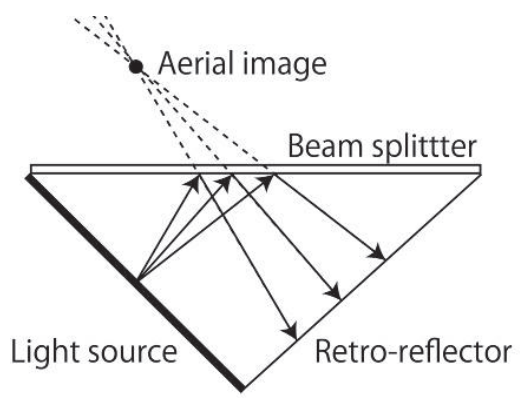

Fig. 1. Principle of AIRR.

\subsection{Polarized AIRR}

In order to increase the brightness of the aerial image, we use polarization modulation. The principle of polarized AIRR is shown in Fig. 2. A reflective polarizer is used for the beam splitter. Also, place a quarter-wave retarder in front of the retro-reflector. The linearly polarized light from the light source transmits the quarter-wave retarder twice before and after being reflected by the retro-reflector. The polarization angle of retro-reflected light is rotated by 90 degrees. After that, the light directed in the incident direction transmits the reflective polarizer and forms an aerial image.

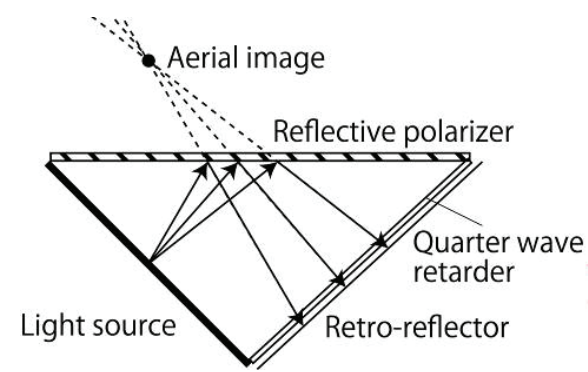

Fig. 2. Principle of polarized AIRR.

2.3 Infinity mirror

The principle of infinity mirror is in shown Fig. 3. The light source is located between a mirror and a half mirror. The virtual images are indicated by (a), (b) and (c) in the figure. Image (a) is the virtual image formed by the mirror directly reflecting the light of the light source. Image (b) is formed by the mirror reflecting the light once reflecting by the half mirror. Image (c) is formed by reflecting the light beam forming (a) again by the half mirror and reflecting it again by the mirror. Similarly, multiple reflections on a mirror and a half mirror form multiple virtual images from one light source.

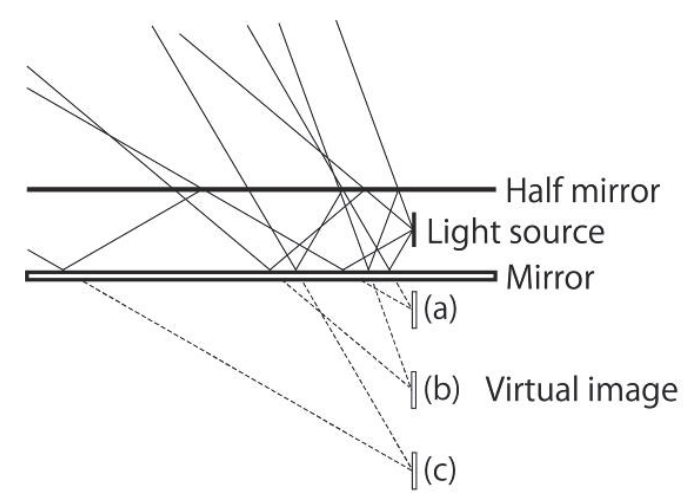

Fig. 3. Principle of infinity mirror. 


\subsection{Multiple aquatic imaging with polarized AIRR} Our proposed method is in shown in Fig. 4. We use a stack of a half mirror, a quarter-wave retarder, a retroreflector instead of the mirror of infinity mirror. Also, a reflective polarizer is placed on the bottom of the tank. Among the multiple reflected light, the light transmitted through the half mirror transmits the quarter-wave retarder twice before and after being retro-reflected. Therefore, the retro-reflected light transmits through the reflective polarizer and form multiple aquatic images.

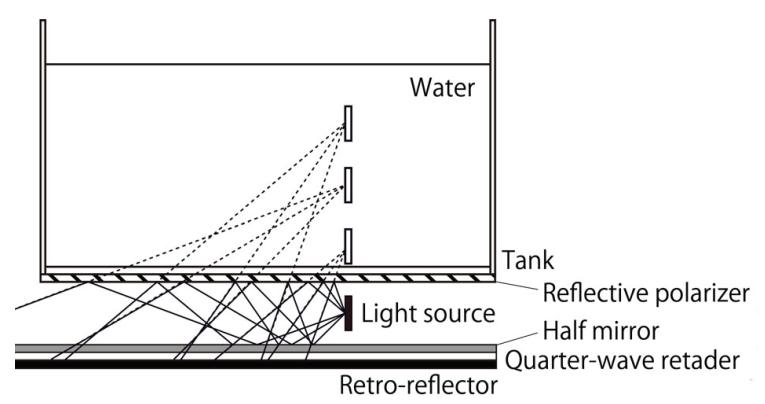

Fig. 4. Multiple aquatic imaging with polarized AIRR.

\section{Simulation result}

In order confirm the feasibility of the proposed optical system, we have conducted optical ray-tracing simulations. Result of a simulation with LightTools is shown in Fig. 5. The result shows that the light rays converge at just above the light source.

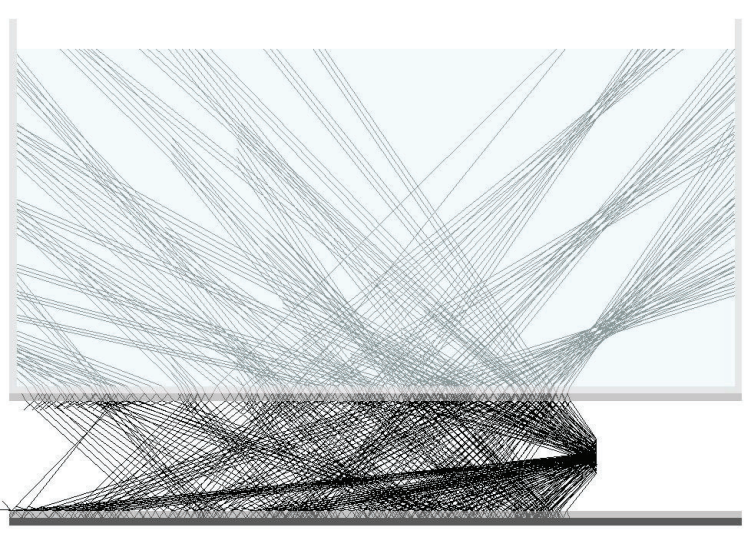

Fig. 5. Simulation result of proposed method.

\section{Experiment}

Multiple aquatic images were formed in the water by use of an LED tape as a light source. Examples of the result is shown in Fig. 6. Also, multiple aquatic images formed by AIRR and polarized AIRR are shown in Fig. 7. In
AIRR, only two rows of aerial images can be seen, whereas in polarized AIRR, five rows of aerial images can be seen.

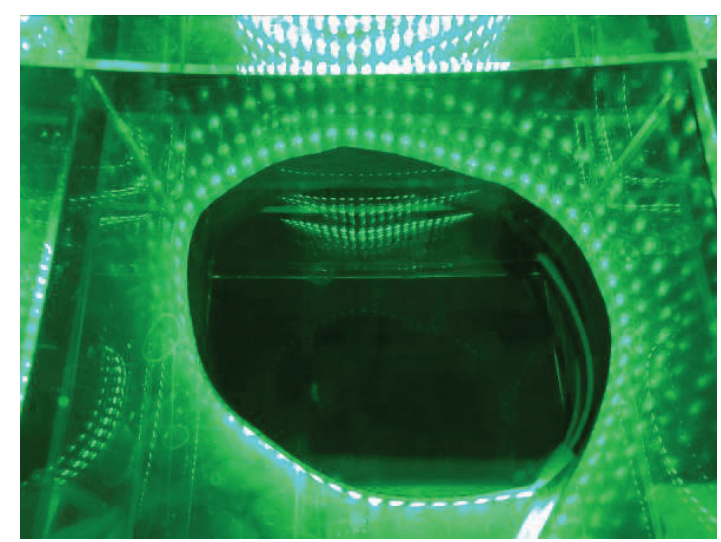

Fig. 6. Multiple aerial images in the water.

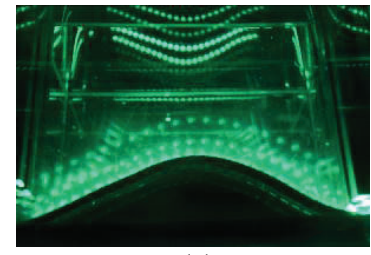

(a)

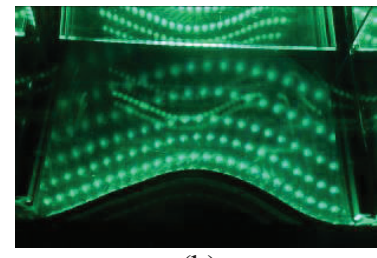

(b)
Fig. 7. Multiple aquatic images with (a)AIRR. (b)polarized AIRR.

\section{Conclusion}

We have successfully formed multiple aquatic images with improved brightness by using polarization modulation.

\section{References}

[1] H. Yamamoto, Y. Tomiyama, and S. Suyama, "Floating aerial LED signage based on aerial imaging by retroreflection (AIRR)", Opt. Express. 22, pp. 26919-26924 (2014).

[2] K. Chiba, M. Yasugi and H. Yamamoto, "Aquatic Multiple Image Formation with Faced Mirror Structure”, IMID 2019 DIGEST, P03-90 (2019).

[3] M. Nakajima, Y. Tomiyama, I. Amimori and H. Yamamoto, "Evaluation Methods of Retro-reflector for Polarized Aerial Imaging by Retro-Reflection", Proc. CLEO Pacific Rim 2015, 25B1-2 (2015).

[4] N. Kurokawa and H. Yamamoto, "Forming multiple aerial images by use of parallel beam splitters with AIRR", Proc. OSJ-OSA Joint Symposia 2016, 30pOD10 (2016). 
LDC2021 | Oral Presentation

$A R, M R, V R, \ldots X R$ technologies 1 - Aerial \&Aquatic Display -

Mon. Apr 19, 2021 2:55 PM - 4:10 PM LDC (Online)

\section{[LDC-2-04] Influence of Diverging Angle of the Light Source on the Image Spot Formed in Water by Use of Retro-reflection}

ODaiki Kudo ${ }^{1}$, Kazunari Chiba ${ }^{1}$, Masaki Yasugi ${ }^{1,2}$, Nao Ninomiya ${ }^{1}$, Hirotsugu Yamamoto ${ }^{1,2}$ (1.Utsunomiya university, 2.JST ACCEL)

The aquatic display has been realized with aerial imaging by retro-reflection (AIRR). We confirmed that the spread of a point image formed in water from a point source placed in the air changes depending on the divergence angle of the light from the source. 


\title{
Influence of diverging angle of the light source on the image spot formed in water by use of retro-reflection
}

\author{
Daiki Kudo ${ }^{1}$, Kazunari Chiba ${ }^{1}$, Masaki Yasugi ${ }^{1,2}$, Nao Ninomiya ${ }^{1}$, Hirotsugu Yamamoto ${ }^{1,2}$ \\ ${ }^{1}$ Utsunomiya university, Yoto 7-1-2, Utsunomiya City, Tochigi, 321-0904, Japan, \\ Tel: +81-28-689-6137, E-mail: hirotsugu@yamamotolab.science, ${ }^{2}$ JST ACCEL
}

\begin{abstract}
The aquatic display has been realized with aerial imaging by retro-reflection (AIRR). We confirmed that the spread of a point image formed in water from a point source placed in the air changes depending on the divergence angle of the light from the source.
\end{abstract}

\section{Introduction}

Aerial Imaging by Retro-Reflection (AIRR) [1] is known as a technique to form an information screen in the air. It has also been reported that biological motions associated with pink noise can induce feeding behaviour in fish. [2] An omnidirectional aerial display was formed using an AIRR and a cone-shaped beam splitter (BS), and the optomotor response of medaka to the omnidirectional aerial display and observed that medaka responded to the aerial image. [3] Our next challenge is to investigate fish reaction to aquatic image that is shown on an information screen in water of an aquarium. However, unlike the image formation in the air, there is an influence of refraction in water, thus it is difficult to accurately detect the position of image formation by observation from outside the water. In the previous paper, we tried to detect the position of aquatic display and size difference between aerial image and it, by using submersible screen [4]. However, although the aquatic image was formed by placing a point light source in the air, it was difficult to form an ideal image due to the large divergence of light rays. In this paper, we showed the possibility of limiting the spread of the point image by limiting the diverging angle of the light emitted from the point source in the air.

\section{Principle}

Light is refracted when it is incident from one media to another. Assuming that light is incident from media A to media $\mathrm{B}$, the angle of incidence is $\mathrm{A}$, the angle of refraction is $B$, and the refractive indices of mediums $A$ and $\mathrm{B}$ are $n_{\mathrm{A}}$ and $n_{\mathrm{B}}$, respectively, the following equation holds.

$$
n_{\mathrm{A}} \sin \theta_{\mathrm{A}}=n_{\mathrm{B}} \sin \theta_{\mathrm{B}}
$$

Fig. 1 shows the optical system to form aquatic image. The aerial image by AIRR is formed at the position of plane symmetry to the BS. By using this property, a real image is formed in water. The BS is placed inside a rectangular box of retro-reflector at an angle of 45 degrees from the bottom. When the light emitted from the light source enters the BS, it is split into transmitted and reflected light, which is reflected by the retro-reflector. The retro-reflected light is transmitted and reflected again by the BS, forming an aquatic image in the water in a direction symmetrical to the light source with respect to the BS. The BS is placed with an offset from the light source. The aquatic image is formed at the distance of na $[\mathrm{mm}]$ from the water tank wall when the offset of the BS is $a[\mathrm{~mm}]$ from the bottom.

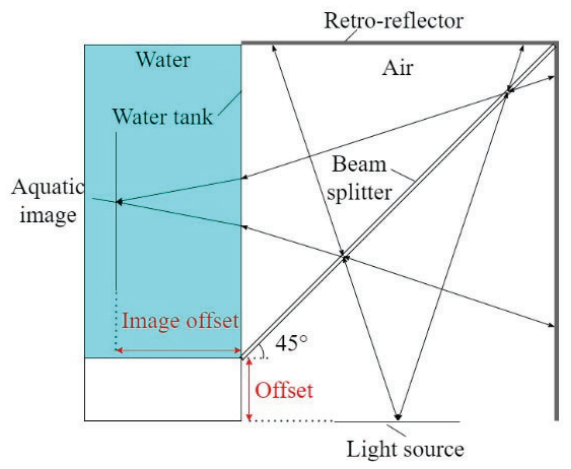

Fig. 1. Optical system to form aquatic image.

\section{Simulation}

In this section, we discuss ray-tracing. The ray-tracing was performed using LightTools 9.0.0. The device is made of retro-reflector and its width is $300 \mathrm{~mm}$, depth is $200 \mathrm{~mm}$ and the height is $150 \mathrm{~mm}$. The BS is $300 \mathrm{~mm}$ wide, $141 \mathrm{~mm}$ high, and $1.00 \mathrm{~mm}$ thick, and is placed at 45.0 degrees to the point source at the bottom. It has a reflectance of $10.0 \%$ and a transmittance of $90.0 \%$. The water is a cube with a side length of $100 \mathrm{~mm}$. The refractive index is set to 1.33 . The holder used to offset the $\mathrm{BS}$ is $300 \mathrm{~mm}$ wide, $100 \mathrm{~mm}$ deep, and $50.0 \mathrm{~mm}$ high, and is made of a material that does not allow light to pass through. The width and height of the detector are $6.00 \mathrm{~mm}$ and the thickness is $1.00 \mathrm{~mm}$. In the present setup, the light emitted from the point source in the air is converged at $66.5 \mathrm{~mm}$ from the surface of the water on the BS side. Considering the use of LED as a point source, the divergence angle values were used from among the following three ranges. This value is a half value angle, so the divergence angle from the light source is doubled. Less than 15.0 (narrow angle light distribution) 15.0 to 30.0 degrees (medium angle light distribution), and 30.0 to 90.0 degrees (wide angle light distribution).

Fig. 2 (a) shows the cross-sectional view of the apparatus, and Fig.2 (b), Fig. 2 (c), and Fig. 2 (d) shows the results of ray tracing at diverging angle of the light 
from the point source is 15.0, 50.0, and 120 degrees, respectively. In addition, Fig. 3 (a), (b), (c) shows the plots of the positions the rays passing over the detector when the diverging angle of the light from the point source is 15.0, 50.0, and 120 degrees, respectively.

Comparing Fig. 2 (b) with Fig. 2 (d), we can see a difference in the convergence position of the light rays. This may also be due to the divergence angle of the rays. As mentioned above, the aerial image is formed in a plane symmetrical to the light source with respect to the BS. When the aerial image is formed, the angle at which the light enters the water is half of the divergence angle of the light from the light source. From equation (1), we can see that the angle of refraction is determined by the angle of incidence, so the position of the image formation changes. Furthermore, comparing Fig. 3(a), Fig. 3 (b), and Fig. 3 (c), it was found that the light focus can be limited by limiting the divergence angle of the light from the point light source. It can also be seen that two focal points are formed from one light source. This is due to the optical path difference caused by the thickness of the BS, which results in double imaging.

\section{Conclusion}

In this paper, we showed the possibility of limiting the spread of a point image by limiting the diverging angle of light emitted from a point source in air. Therefore, when a display is used as a light source to form aquatic display, it is considered possible to form aquatic display with high resolution by using a viewing angle control film.

\section{Acknowledgment}

A part of this work was supported by JST/ACCEL (grant no. JPMJAC1601) and JSPS KAKENHI (19H04155, 20H05702).

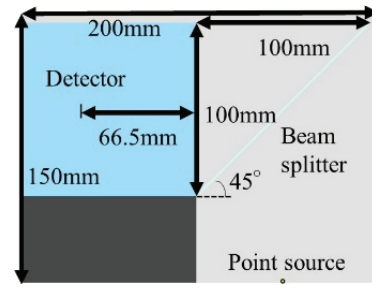

(a)

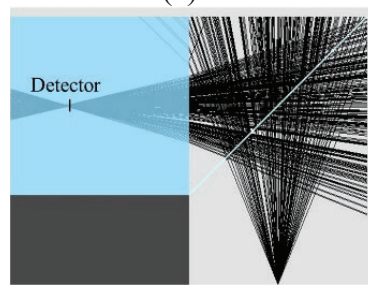

(c)

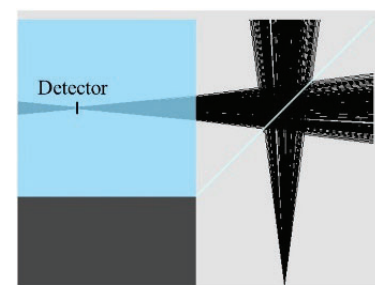

(b)

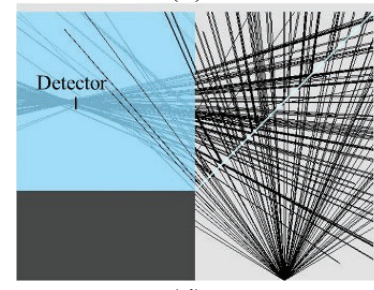

(d)
Fig. 2. The (a) cross-sectional view of the apparatus, and the results of ray tracing when the diverging angle of (a) 15.0, (b) 50.0, and (c) 120 degrees, respectively.

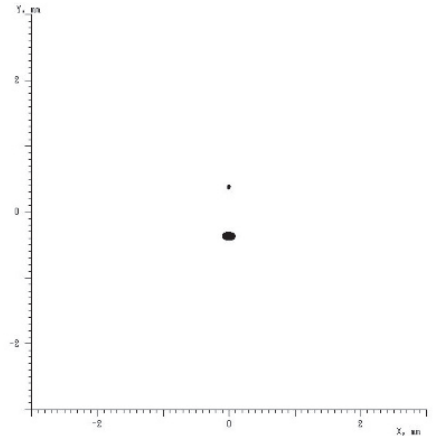

(a)

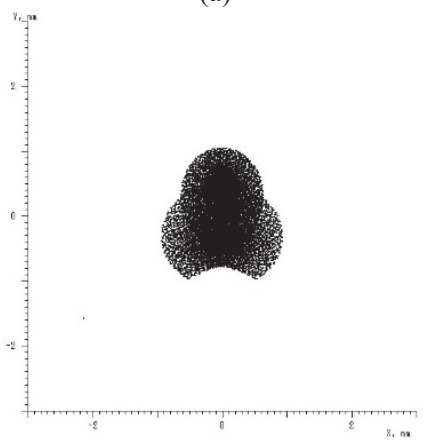

(b)

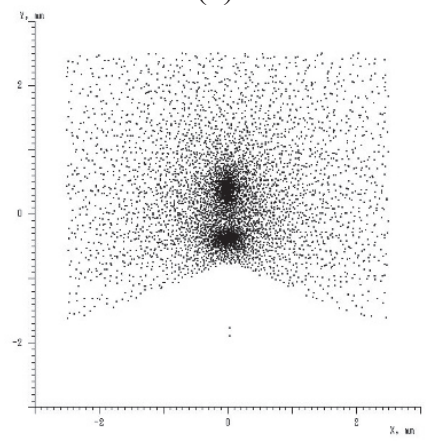

(c)

Fig. 3. The plots of the positions the rays passing over the detector when the diverging angle of the light from the point source is (a) 15.0, (b) 50.0, and (c) 120 degrees, respectively.

\section{References}

[1] H. Yamamoto, Y. Tomiyama, and S. Suyama, "Floating aerial LED signage based on aerial imaging by retroreflection (AIRR)”, Opt. Express 22, 26919-26924 (2014).

[2] W. Matsunaga and E. Watanabe, "Visual motion with pink noise induces predation behaviour", Sci. Rep. 2, 219 (2012).

[3] E. Abe, M. Yasugi, H. Takeuchi, E. Watanabe, Y. Kamei, and H. Yamamoto, "Development of omnidirectional aerial display with aerial imaging by retro-reflection (AIRR) for behavioral biology experiments," Opt. Rev. 26, 221-229 (2019).

[4] D. Kudo, K. Chiba, M. Yasugi, and H. Yamamoto, "Measurement of Screen Position of an Aquatic Display", in The 27th International Display Workshops (IDW'20), pp. 265-268 (2020). 
LDC2021 | Oral Presentation

$A R, M R, V R, \ldots X R$ technologies 2 - Viewing angle in AR/VR display and devices -

Tue. Apr 20, 2021 10:00 AM - 11:30 AM LDC (Online)

Click to the Online Room

[LDC-3-01 (Invited)] Expanding Field-of-view in Head-mounted Displays Considering Characteristics of Vision

OWataru Yamada ${ }^{1}$, Hiroyuki Manabe ${ }^{1}$ (1.NTT DOCOMO, INC.)

10:00 AM - 10:30 AM

[LDC-3-02] Analysis on Viewing angle in Aerial Display by Use of an LED Panel Covered with Apertured Retro-Reflector

ODaiki Nishimura ${ }^{1}$, Masaki Yasugi ${ }^{1,2}$, Hirotsugu Yamamoto ${ }^{1,2}$ (1.Utsunomiya University, 2.JST, ACCEL)

10:30 AM - 10:45 AM

[LDC-3-03] Increasing luminance of aerial image formed with AIRR by use of dual transparent spheres

OKengo Fujii ${ }^{1}$, Satoshi Maekawa ${ }^{2}$, Hirotsugu Yamamoto ${ }^{1,3}$ (1.Utsunomiya

University, 2.Parity Innovations, 3.JST ACCEL)

10:45 AM - 11:00 AM

[LDC-3-04 (Invited)] Compact Full-color Laser Beam Projectors Based on Waveguide-type RGB Multiplexers

OToshio Katsuyama ${ }^{1}$, Akira Nakao ${ }^{1}$, Shoji Yamada ${ }^{1}$, Osamu Kawasaki ${ }^{2}$, Kazuki Iwabata $^{2}$, Koichi Horii ${ }^{2}$, Akira Himeno ${ }^{1,2}$ (1.University of Fukui, 2.SEIREN KST Corp.)

11:00 AM - 11:30 AM 
LDC2021 | Oral Presentation

$A R, M R, V R, \ldots X R$ technologies 2 - Viewing angle in AR/VR display and devices -

Tue. Apr 20, 2021 10:00 AM - 11:30 AM LDC (Online)

\section{[LDC-3-01 (Invited)] Expanding Field-of-view in Head-mounted Displays Considering Characteristics of Vision}

OWataru Yamada ${ }^{1}$, Hiroyuki Manabe ${ }^{1}$ (1.NTT DOCOMO, INC.)

Field of view (FOV) is one of the key parameters for the performance of head-mounted displays. Focusing on the differences between central vision and peripheral vision, we developed a technique for expanding the FOV of a head-mounted display at low cost by using lenses with different levels of magnification. 


\title{
Expanding Field-of-view in Head-mounted Displays Considering Characteristics of Vision
}

\author{
Wataru Yamada, Hiroyuki Manabe* \\ NTT DOCOMO, INC, Tokyo, Japan, wataruyamada@acm.org,
}

\begin{abstract}
Field of view (FOV) is one of the key parameters for the performance of head-mounted displays. Focusing on the differences between central vision and peripheral vision, we developed a technique for expanding the FOV of a head-mounted display at low cost by using lenses with different levels of magnification.
\end{abstract}

\section{Introduction}

A head-mounted display (HMD) is a type of display device worn on the head to present images or videos to the user. Technological improvements such as better displays, greater processing power, and higher mobility have made it possible for HMD with high immersion to become popular consumer products. Many HMD products for virtual reality (VR) have been released, and many applications are being created.

HMDs for VR can be roughly divided into two types. The first type (hereinafter referred to as "integrated HMD") is an HMD having processing units, displays, and lenses like STAR VR [1]. Integrated HMDs can achieve high performance due to purpose-built modules such as multiple displays, fast processing units, and complex optical parts. For example, STAR VR [1] has $210^{\circ}$ horizontal field of view (FOV), $5 \mathrm{~K}$ resolution, and 90 frames per second by adopting special modules such as two large displays and complex Fresnel lenses.

The second type (hereinafter referred to as "add-on HMD") provides only a case and lenses as in the Google Cardboard [2] viewer. It can be used as an HMD by inserting a smartphone into the viewer. The greatest advantage of an add-on HMD is that it can be achieved at a very low cost since it enables users to use their own smartphones. On the other hand, because only the display, sensors and CPU of the smartphone can be used in add-on HMDs, the performance of an add-on HMD is inferior to that of an integrated HMD that can mount displays having specialized modules.

The performance of an HMD can be evaluated by various criteria, but an important one is FOV, an index of the user's range of vision when wearing an HMD. Although a wide FOV can give rise to discomfort or nausea, i.e., VR sickness in a VR environment, it can also deepen a sense of immersion and presence [3]. Thus, a number of researches had tried to make FOV wider to enhance user experience in VR environments; however, technical difficulties in producing commercial devices with wide FOV (e.g., complicated optics, increased weight, etc.) often limit the available FOV [4].
Robert et al. [4] focused on the fact that peripheral vision has a much lower resolution than foveal vision. In order to expand FOV, they proposed to fill the periphery of the HMD with a low-resolution, high-contrast array of diffused, colored light-emitting diode (LED) lights, whose driving signals are tightly coupled to the content presented on the device. Their approach can expand FOV without complicated optics. Unfortunately, it is still difficult to apply it to add-on HMDs because of the additional costs; it needs various devices such as a lot of colored LED lights, processing units, battery, and diffuser panel.

Thus, we have proposed a technique for expanding the FOVof an HMD at low cost with a lightweight configuration by focusing on human FOV characteristics [5]. The proposed method consists of a special lens that combines a convex lens with normal focal length and a curved Fresnel lens with a shorter one. The convex lens shows a high-resolution image in foveal vision. Moreover, the Fresnel lens around the convex lens fills the peripheral vision with a blurred image. The result is analogous to wearing glasses for near-sighted eyes. The glasses yield a clear image inside of the lens but the image outside of it is blurred. However, without this outer blurred image, we feel as if FOV is narrowed.

The key idea of displaying a high-resolution image only in the central FOV, taking into account human visual field characteristics, is common to the method using LED arrays proposed by Robert et al [4]. However, the proposed method has the advantages such as low weight and cost and high versatility, because our method does not require additional electronic components. For this reason, the proposed method supports not only integrated HMD but also add-on HMD unlike the method proposed by Robert et al [4]. Besides, the proposed method also does not require a new software. In other words, the existing contents and VR applications is available in our system. We believe that the proposed method is practical due to its low cost, lightweight, and easy implementation.

\footnotetext{
* Currently, he is with Shibaura Institute of Technology.
} 


\section{Implementation}

Figure 1 shows the prototype of the proposed method. The prototype consists of $30.4 \mathrm{~mm}$ diameter convex lens with a $33 \mathrm{~mm}$ focal length and curved Fresnel lens. The Fresnel lens has a focal length of a $33 \mathrm{~mm}$ at the border with a convex lens, and the focal length gradually becomes shorter as it approaches the edge.

The system that we have proposed [5] used a flat Fresnel lens. The reason why the Fresnel lens is curved in the current prototype is to cover the user's eyes and allow lights to reach a wider area of the user's vision.

The convex lens and the Fresnel lens are made as one piece by injection molding. The material of the lens is acrylic resin and its weight is only $15.4 \mathrm{~g}$. The distance between the surface of the lenses and a screen is $28.5 \mathrm{~mm}$. In the design, there is also a distance of $10.5 \mathrm{~mm}$ between the surfaces of the user's eye and the convex lens.

We installed an iPhone XS max that has a 6.5-inch display (2688 x 1242 pixel) in the prototype and captured a close-up image to confirm the view of the proposed method. Figure 2 is a close-up of the prototype. The image is taken focused on the screen, through the convex lens.

The screen is clearly visible through the convex lens. In contrast, the area of the Fresnel lens is blurred because the screen through the Fresnel lens is not in the focus. Note that the focal length of the Fresnel lens is much shorter than the convex lens. The proposed method assumes that the user always focuses on the screen through the convex lens in actual use. In other words, the user moves range of vision by moving their head.

A user sees both clear and blurred images in actual use as Fig. 2 shows. This blurry peripheral region widens the FOV of the HMD. by adding a Fresnel lens, the overall FOV increases to over 140 degrees.

\section{Conclusion}

We proposed a method to widen the FOV of HMDs by using lenses with different focal lengths for central and peripheral vision. In the proposed method, a clear image is displayed in the center of the screen, and the most of peripheral vision is filled with blurred images. We implemented a prototype to confirm the characteristics of the proposed method. The total image area is wider than that of the images produced using an only convex lens, confirming that the FOV was widened. The proposed method, which does not require additional electronic devices, is low cost and can be made lightweight. Therefore, it can be applied to various HMDs including Add-on HMDs.

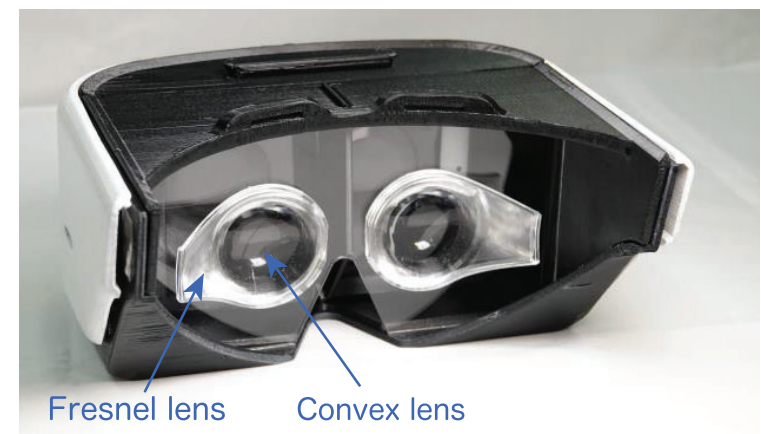

Figure 1. Images of our prototype. Proposed lenses consist convex lenses and curved Fresnel lenses.

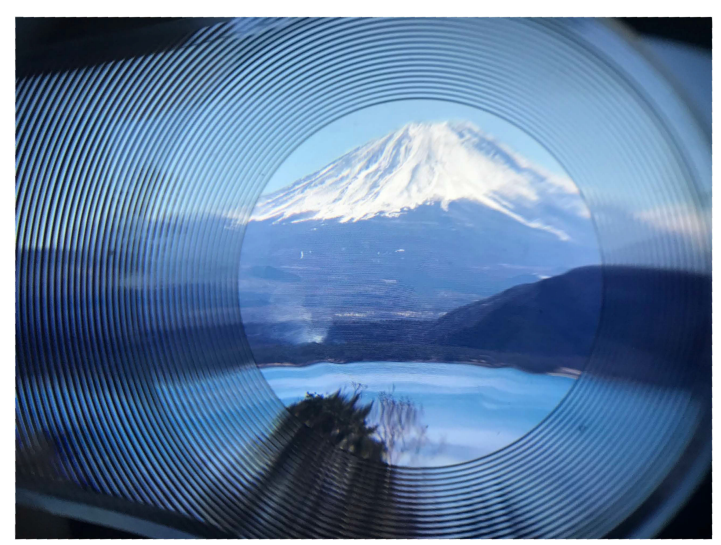

Figure 2. Close-up image from the inside of the prototype.

\section{References}

[1] InfinitEye. StarVR Tech Specs, http://www.starvr.com/, Retrieved February 21, 2021.

[2] Google Cardboard, https://vr.google.com/cardboard/, Retrieved February 21, 2021.

[3] J. J.- Lin, H. B. L. Duh, D. E. Parker, H. Abi-Rached, and T. A. Furness. 2002. Effects of field of view on presence, enjoyment, memory, and simulator sickness in a virtual environment. In Proceedings IEEE Virtual Reality 2002, 164-171.

[4] Robert Xiao and Hrvoje Benko. 2016. Augmenting the Field-of-View of Head-Mounted Displays with Sparse Peripheral Displays. In Proceedings of the 2016 CHI Conference on Human Factors in Computing Systems. Association for Computing Machinery, New York, NY, USA, 1221-1232.

[5] Wataru Yamada and Hiroyuki Manabe. 2016. Expanding the Field-of-View of Head-Mounted Displays with Peripheral Blurred Images. In Proceedings of the 29th Annual Symposium on User Interface Software and Technology (UIST '16 Adjunct), 141-142. 
LDC2021 | Oral Presentation

$A R, M R, V R, \ldots X R$ technologies 2 - Viewing angle in AR/VR display and devices -

Tue. Apr 20, 2021 10:00 AM - 11:30 AM LDC (Online)

\section{[LDC-3-02] Analysis on Viewing angle in Aerial Display by Use of an LED} Panel Covered with Apertured Retro-Reflector

ODaiki Nishimura ${ }^{1}$, Masaki Yasugi ${ }^{1,2}$, Hirotsugu Yamamoto ${ }^{1,2}$ (1.Utsunomiya University, 2.JST, ACCEL)

This paper proposes an analytical model on the viewing angle of the aerial image formed over an LED panel. Our optical system forms aerial signage over a specially fabricated LED panel. A half mirror is placed in front of the LED panel that is covered with retro-reflector with square-shaped holes. 


\title{
Analysis on Viewing angle in Aerial Display by Use of an LED Panel Covered with Apertured Retro-Reflector
}

\author{
Daiki Nishimura ${ }^{1}$, Masaki Yasugi ${ }^{1,2}$, Hirotsugu Yamamoto ${ }^{1,2}$ \\ ${ }^{1}$ Utsunomiya University, 7-1-2 Yoto, Utsunomiya City, Tochigi, 321-0904 Japan, \\ ${ }^{2}$ JST, ACCEL, 7-1-2 Yoto, Utsunomiya City, Tochigi, 321-0904 Japan, \\ +81 28689 7074, hirotsugu@yamamotolab.science.
}

\begin{abstract}
This paper proposes an analytical model on the viewing angle of the aerial image formed over an LED panel. Our optical system forms aerial signage over a specially fabricated LED panel. A half mirror is placed in front of the LED panel that is covered with retro-reflector with square-shaped holes.
\end{abstract}

\section{Introduction}

Aerial imaging by retro-reflection (AIRR) has been proposed as a method to form an aerial image for a wide range of viewing angle [1]. The conventional optical design of AIRR requires a large foot space to install the large aerial display system. Recently, to solve this problem, we have realized the novel optical design that forms an aerial image without moiré that can be observed from the front of the LED panel [2] and the analytical model to formulate the relationships between the aperture ratio of the retro-reflector and the luminance of the aerial image [3].

In this paper, we propose an analytical model to formulate the relationships between the seize of display area of LED panel and the viewing angle of the aerial image. Where viewing angle is the right and left angle at which the entire aerial image can be viewed when viewed from the front.

2. Optical system for aerial signage over an LED panel Fundamental structure to form the aerial image in front of an LED panel is shown in Fig. 1. A retro-reflector that has square apertures covers the LED panel so that LED lamps in a light scattering package are placed in the apertures. Then, a half mirror is placed in parallel with the LED panel. The light from the LED panel goes to the half mirror and splits in reflected light and transmitted light. The reflected light impinges the retro-reflector and goes back to the half mirror after the retro-reflection. The light splits again on the half mirror. The transmitted light converges to the position of plane symmetry of the light source in respect to the half mirror.

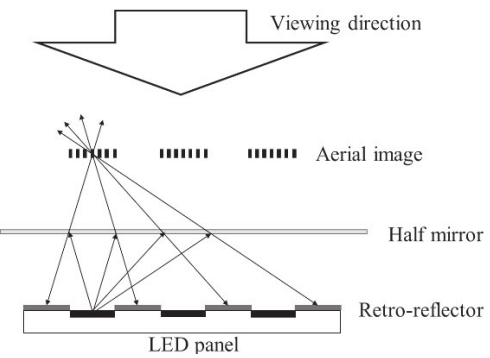

Fig. 1. Optical system to form aerial signage by use of patterned retro-reflector.

\section{Developed prototype aerial display by use of an LED panel covered with apertured retro-reflector}

We developed a prototype for experimental confirmation of our optical system. A 6-mm-pitch $32 \times 32$ pixels LED panel was used for the light source. The space between the LED panel and the half mirror was $100 \mathrm{~mm}$. The retroreflector with square-shaped apertures and the aerial image viewed from the front in the experiment are shown in Fig. 2. We used the retro-reflector produced by Nippon Carbide Industries (RF-Ax). In this setting, the lightsource LED panel and aerial image were visible to the observer and moiré was not observed on aerial images.

We have confirmed that the rays converged on the screen when we placed a screen on the position of plane symmetry of the light source with respect to the beam splitter, as shown in Fig. 3 (a). When the screen was moved frontward and backward, the images on the screen were blurred, as shown in Fig. 3 (b) and (c) respectively. The space between the LED panel and the screen was (a) $200 \mathrm{~mm}$, (b) $210 \mathrm{~mm}$, (c) $190 \mathrm{~mm}$ in Fig. 4.
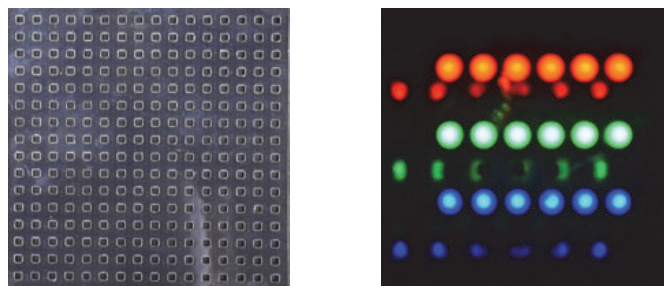

Fig. 2. A retro-reflector with square-shaped holes (left) and an aerial image viewed in front (right).

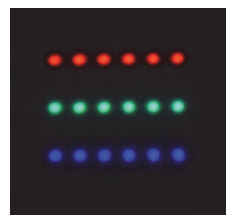

(a)

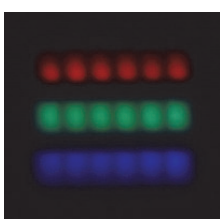

(b)

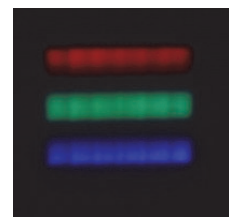

(c)
Fig. 3. Observations of the formed aerial images on a screen when the screen is placed (a) on the light-converging position, (b) after the light-converging position, and (c) before the light-converging position. 


\section{Analyzing viewing angle of the aerial image}

We have analyzed the viewing angle of the aerial image. An analytical model for the viewing angle of our optical system is shown in Fig. 4, where $X_{m}$ is one side length of the LED panel; $X_{n}$ is one side length of light emitting region; and $Y$ is the space between the LED panel and the half mirror. The region of interest (ROI) is supposed as a square image composed of an even number of light emitting pixels at the center of the LED panel. The viewing angle changes according to the size of the display area. Pixels layout of the LED panel is shown in Fig. 5. Were $P_{D}$ is the pitch of the LED panel and $P_{D} / 2$ is one side length of an LED lamp. The number of pixels on one side of the LED panel is $2 m$ and the number of pixels on one side of the ROI is $2 n$. In Fig. 4 , we define the base axis perpendicular to the half mirror surface. The angle $\theta$ is the viewing angle of aerial image and is expressed by the following equations:

$$
\begin{array}{ll}
\theta=\tan ^{-1} \frac{X_{m}-X_{n}}{4 Y} & \left(0<\theta<\frac{\pi}{2}\right), \\
X_{m}=(4 m+1) \frac{P_{D}}{2} & (m=1,2,3, \cdots),
\end{array}
$$

and

$$
X_{n}=(4 n-1) \frac{P_{D}}{2} \quad(n=1,2,3, \cdots) \text {. }
$$

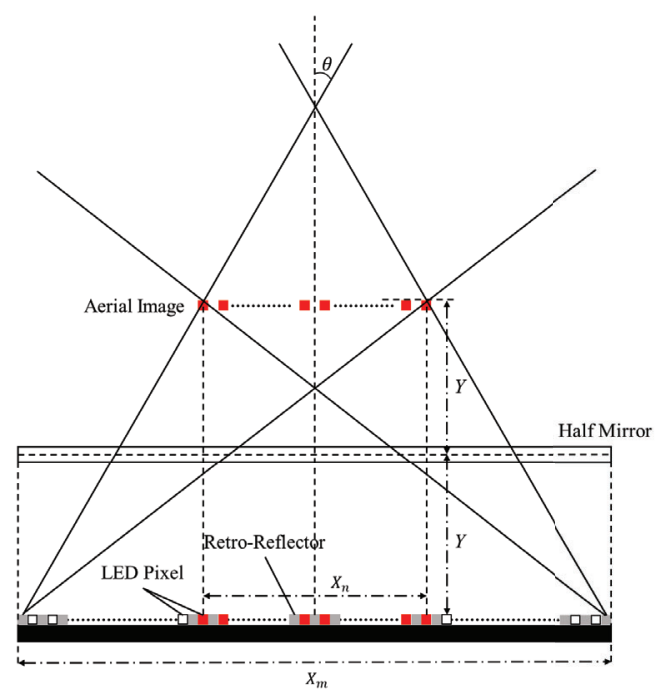

Fig. 4. Viewing angle of aerial image.

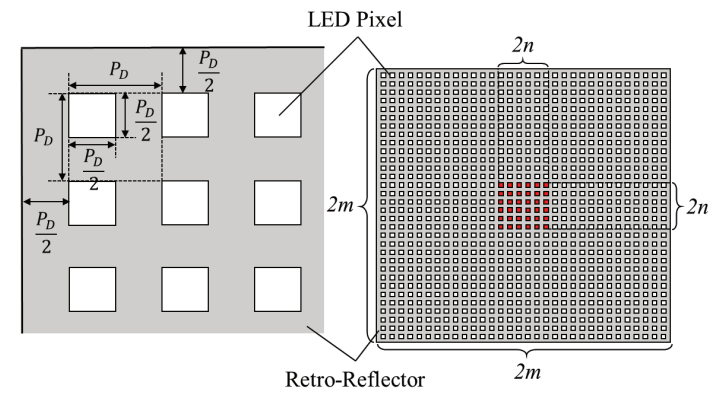

Fig. 5. Pixels layout of the LED panel covered with apertured retro-reflector.
In our prototype aerial display, $P_{D}$ is $3 \mathrm{~mm}$ and $m$ is 16. When $\frac{Z}{X_{m}}$ is $0.5,1$ and 1.5 , and $0 \leq \frac{n}{m} \leq 1$, viewing angle of aerial image is shown in Fig. 6. Larger ROI on the LED panel makes the viewing angle smaller.

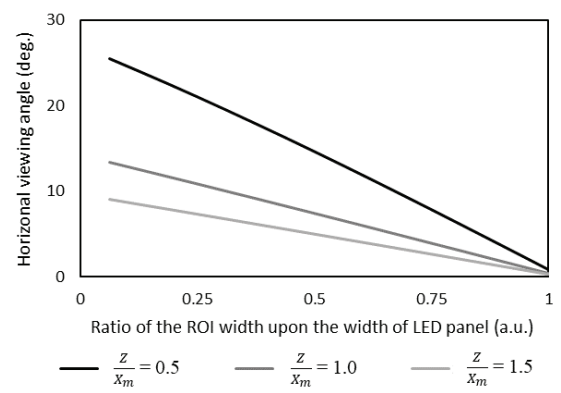

Fig. 6. Relationships between ratio of ROI upon the display area and the viewing angle of the aerial image.

\section{Conclusion}

We have successfully formed aerial images without moiré in front of the LED panel, by placing a beam splitter in front of the LED panel that is covered with apertured retro-reflector. Showing an aerial image right in front of an LED panel reduces the foot space, which is suitable for a large-scale installation. Thus, the proposed optical system is suitable for the aerial signage with large screen for traffics, advertisement, amusements, and so on.

Since our proposed optical system can be installed in a small space, it will be useful for aerial interface in hospitals and public areas. By using aerial display as noncontact display, it is possible to prevent the spread of infectious diseases because we don't have to touch where others touched.

We have analyzed the viewing angle of the aerial image formed by our optical design. The size of display area of LED panel and the viewing angle of the aerial image are correlated. The next step of this study is to measure of the viewing angle of the aerial images by use of the prototype, and confirm measured results are consistent with our analysis.

A part of this work was supported by JST ACCEL Grant Number JPMJAC1601, Japan. A part of this work was supported by JST/ACCEL (grant no. JPMJAC1601) and JSPS KAKENHI (19H04155, 20H05702).

\section{References}

[1] H. Yamamoto, Y. Tomiyama, and S. Suyama, "Floating aerial LED signage based on aerial imaging by retroreflection (AIRR)," Opt. Express 22, 26919 (2014).

[2] D. Nishimura, M. Yasugi, and H. Yamamoto, "Forming Aerial Signage in Front of LED Panel by Use of RetroReflector with Square-Shaped Holes," Proc. LDC'20, LDC9-04 (2020).

[3] D. Nishimura, M. Yasugi, and H. Yamamoto, "Analysis on Optimal Aperture Ratio in Aerial Display by Use of an LED Panel Covered with Apertured Retro-Reflector," Proc. IP'20, PA10 (2020). 
LDC2021 | Oral Presentation

$A R, M R, V R, \ldots X R$ technologies 2 - Viewing angle in AR/VR display and devices -

Tue. Apr 20, 2021 10:00 AM - 11:30 AM LDC (Online)

\section{[LDC-3-03] Increasing luminance of aerial image formed with AIRR by} use of dual transparent spheres

OKengo Fujii ${ }^{1}$, Satoshi Maekawa ${ }^{2}$, Hirotsugu Yamamoto ${ }^{1,3}$ (1.Utsunomiya University, 2.Parity Innovations, 3.JST ACCEL)

We propose a method to form aerial display by installing two transparent spheres in the light path of AIRR. By proposed method, we confirmed by simulation that the luminance of the aerial image was increased. 


\title{
Increasing luminance of aerial image formed with AIRR by use of dual transparent spheres
}

\author{
Kengo Fujii ${ }^{1}$, Satoshi Maekawa ${ }^{2}$, Hirotsugu Yamamoto ${ }^{1,3}$ \\ ${ }^{1}$ Utsunomiya university, Yoto 7-1-2, Utsunomiya City, Tochigi, 321-0904, japan, \\ Tel: +81-28-689-6137,E-mail: k fujii@yamamotolab.science \\ ${ }^{2}$ Parity Innovations, ${ }^{3}$ JST ACCEL
}

\begin{abstract}
We propose a method to form aerial display by installing two transparent acrylic spheres in the light path of AIRR device. By combining the transparent spheres, we confirmed by simulation that the luminance of the aerial image was increased more than the conventional AIRR.
\end{abstract}

\section{Introduction}

The research and development of methods for threedimensionally presenting information have been carried for their application to advertising and amusement, but their use as a touchless interface is currently attracting attention from the perspective of preventing the spread of infectious diseases. Aerial imaging by retro-reflection (AIRR) $[1,2]$ is one of such techniques. AIRR makes floating real images in the mid-air that can be seen by the naked eye. The configuration of conventional AIRR requires a light source, a beam splitter, and a retroreflector.

Our previous study proposed a novel steganography that utilizes AIRR and two transparent spheres in the same size. The proposed method used one of the transparent spheres as a key to form an aerial image that was formed with AIRR [3]. In conventional AIRR configuration, the size of retro-reflector is required to be the same as or larger than the one of a light source. In proposed method, the transparent sphere acts like a ball lens, focusing the light from the display to the retroreflector. This made it possible to reduce the area of retro-reflector required for aerial imaging [4]. Here, the light rays are focused by the transparent sphere, which may reduce the loss of light rays and increase the luminance of the aerial image.

In this paper, we use simulations to confirm that aerial image formed by AIRR using planesymmetrically-placed two transparent spheres increase luminance more than conventional AIRR.

\section{Principle of AIRR with two transparent spheres}

Fig. 1 shows the diagram of conventional AIRR. This setup consists of a light source, a beam splitter, and a retro-reflector. The beam splitter reflects rays from the light source. The reflected rays are retro-reflected, that is, reflected reversely at the incident positions on the retroreflector. The retro-reflected rays are converged to the plane-symmetrical position of the light source with respect to the beam splitter.

Fig. 2 shows the diagram of our proposed method where the two same transparent spheres are placed plane-symmetrically regarding the beam splitter. The light emitted from the light source passes through the transparent sphere 1 with refraction, and then the light is reflected by the beam splitter and enters to the retroreflector. The retro-reflected light passes through the beam splitter and is refracted by the transparent sphere 2 , which forms aerial image at the plane-symmetrical position of the light source with respect to the beam splitter. Fig. 3 shows the observation results of aerial images using the assembled device.

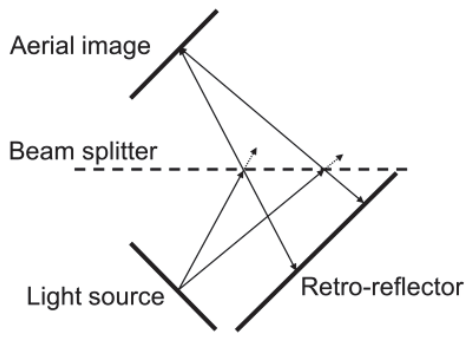

Fig. 1. Principle of aerial imaging by retro-reflection (AIRR).

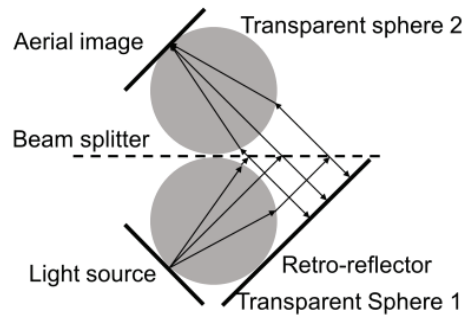

Fig. 2. Principle of imaging an aerial image formed by AIRR with two transparent spheres.

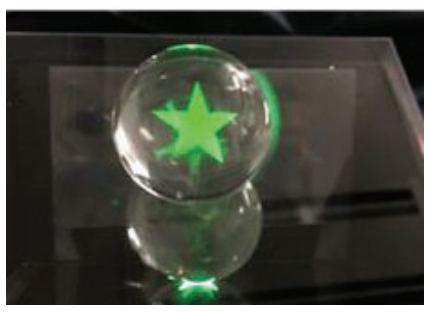

Fig. 3. Image of an aerial image formed by AIRR with two transparent spheres. 


\section{Simulation results for aerial imaging}

Fig. 4 shows the diagram of the setup for ray tracing in AIRR with transparent spheres. Ray tracing was performed using Light tools 9.0.0. The surface light source is a square of $50 \mathrm{~mm}$ square and is placed at an angle of 45 degrees to the beam splitter. The directivity of the surface light source (called the target sphere in Light Tools) is 45 degrees. Transparent Sphere 1 and the surface light source are touching at the center point of the surface light source. The size of the retro-reflector is $110 \mathrm{~mm}$ square, which is enough to receive the light reflected from the surface light source through the transparent sphere to the beam splitter. In this simulation, the retro-reflector has retro-reflection without spreading and attenuation. The beam splitter is a square of $200 \mathrm{~mm}$. It has the characteristics of a half-mirror. The two transparent spheres are made of acrylic and have the same diameter of $70 \mathrm{~mm}$. They were placed symmetrically at the beam splitter and were tangent at the center of the beam splitter.

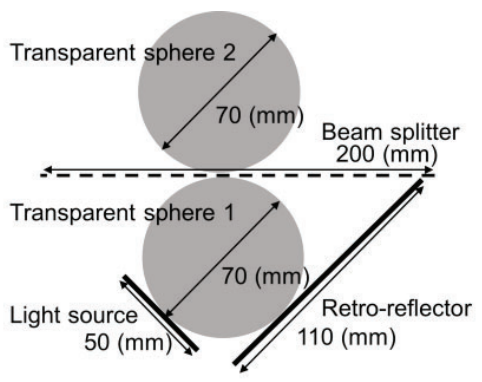

Fig. 4. Simulation settings.

We performed ray-tracing simulations from the time the light is emitted from the light source until it forms the aerial image. The parameters of each element are the same as those shown in Fig. 4. The detector is placed at the position of plane-symmetry with the light source and the beam splitter.

Fig. 5 (a) shows the results of ray tracing simulation of conventional AIRR. Fig. 5 (b) show the simulation results of illuminance distribution at the imaging surface of an aerial image. It can be seen that an aerial image with a size of about $50 \mathrm{~mm}$ square is formed. The highest luminance value was $23.1 \mathrm{kLux}$.

Fig. 6 (a) shows the results of ray tracing simulation of AIRR using two transparent spheres arranged in plane-symmetry with a beam splitter. Fig. 6 (b) show the simulation results of illuminance distribution at the imaging surface. The highest luminance value was 49.5 kLux. From the simulation results, it was confirmed that the combination of the transparent spheres increased the luminance of the aerial image more than the conventional AIRR.

Comparing Fig. 5 (a) and Fig. 6 (a), it can be seen that less light enters the retro-reflector directly from the light source in Fig. 6(a) than in Fig. 5(a). This indicates that by using the transparent spheres, more light from the light source can be used to form an aerial image.

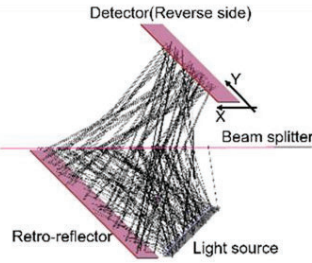

Fig. 5. ( ${ }^{\text {Results }}$ of (a) ray tracing and (b) luminance distribution simulation of the light emitted from the light source until it forms an aerial image in conventional AIRR.

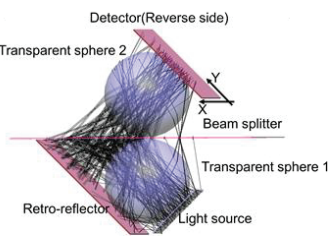

(a)

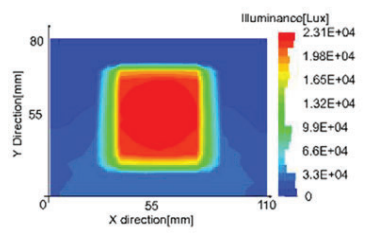

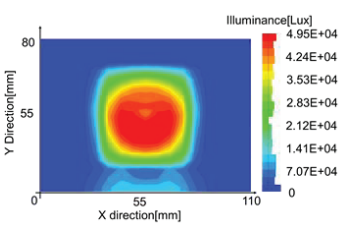

(b)
Fig. 6. Results of (a) ray tracing and (b) luminance distribution simulation of the light emitted from the light source until it forms an aerial image in proposed method using two transparent spheres.

\section{Conclusion}

We confirmed by simulation that AIRR using the transparent spheres can form aerial images with increased luminance more than conventional AIRR.

\section{Acknowledgment}

A part of this work was supported by JST/ACCEL (grant no. JPMJAC1601) and JSPS KAKENHI (19H04155, 20H05702).

\section{References}

[1] H. Yamamoto, Y. Tomiyama, and S. Suyama, "Floating aerial LED signage based on aerial imaging by retroreflection (AIRR)," Opt. Express, Vol. 22, 26919-26924 (2014).

[2] M. Nakajima, K. Onuki, I. Amimori, and H. Yamamoto, "Polarization State Analysis for Polarized Aerial Imaging by Retro-Reflection (PAIRR)," Proc. IDW, Vol. 22, 429432 (2015)

[3] K. Fujii, S. Ito, S. Maekawa, and H. Yamamoto, "Steganography by use of a clear sphere as a key for decoding a concealed aerial image formed with AIRR," Proc. IP'17, 21PM-1-3 (2017).

[4] K. Fujii and H. Yamamoto, "Aerial Display on a Clear Sphere with Aerial Imaging by Retro-Reflection," Proc. MOC2019, 222-223 (2019). 
LDC2021 | Oral Presentation

$A R, M R, V R, \ldots X R$ technologies 2 - Viewing angle in AR/VR display and devices -

Tue. Apr 20, 2021 10:00 AM - 11:30 AM LDC (Online)

\section{[LDC-3-04 (Invited)] Compact Full-color Laser Beam Projectors Based on Waveguide-type RGB Multiplexers}

OToshio Katsuyama ${ }^{1}$, Akira Nakao ${ }^{1}$, Shoji Yamada ${ }^{1}$, Osamu Kawasaki ${ }^{2}$, Kazuki Iwabata ${ }^{2}$, Koichi Horii ${ }^{2}$, Akira Himeno $^{1,2}$ (1.University of Fukui, 2.SEIREN KST Corp.)

Compact full-color laser sources and laser beam scanning modules are demonstrated. Those are based on waveguide-type RGB multiplexers. In principle, their output laser beams are perfectly aligned, which inevitably leads to clear projection images. Thus, compact imaging projectors are constructed, which are applicable to a variety of imaging fields. 


\title{
Compact Full-color Laser Beam Projectors Based on Waveguide-type RGB Multiplexers
}

\author{
Toshio Katsuyama ${ }^{1}$, Akira Nakao ${ }^{2}$, Shoji Yamada ${ }^{2}$, Osamu Kawasaki ${ }^{3}$, Kazuki Iwabata ${ }^{3}$, Koichi Horii ${ }^{3}$, Akira Himeno ${ }^{2,3}$ \\ ${ }^{1}$ Headquarters for Innovative Society-Academia Cooperation (UF-HISAC), University of Fukui, \\ Bunkyo 3-9-1, Fukui City, Fukui 910-8507, Japan, +81-776-27-8956, t-katsu@u-fukui.ac.jp, \\ ${ }^{2}$ Headquarters for Innovative Society-Academia Cooperation (UF-HISAC), University of Fukui, ${ }^{3}$ SEIREN KST Corp.
}

\begin{abstract}
Compact full-color laser sources and laser beam scanning modules are demonstrated. Those are based on waveguide-type $R G B$ multiplexers. In principle, their output laser beams are perfectly aligned, which inevitably leads to clear projection images. Thus, compact imaging projectors are constructed, which are applicable to a variety of imaging fields.
\end{abstract}

\section{Introduction}

Compact image projectors such as eyewear displays have been extensively investigated [1]. In particular, laser projectors based on a beam scanning method have occupied an important position because of their size compactness and clearness of the image [2, 3].

Recently, we have developed a new waveguide-type redgreen-blue (RGB) laser beam multiplexer, which is integrated with semiconductor lasers [4-6]. This integrated laser source is so small that it can be applied to the full-color laser beam scanning projectors including eyewear displays [7].

Here, we review our waveguide-type RGB multiplexers, laser sources, projectors, and their candidate applications.

\section{Waveguide-type RGB multiplexers}

The waveguide-type RGB laser beam multiplexer is schematically shown in Fig.1 (a). It consists of three

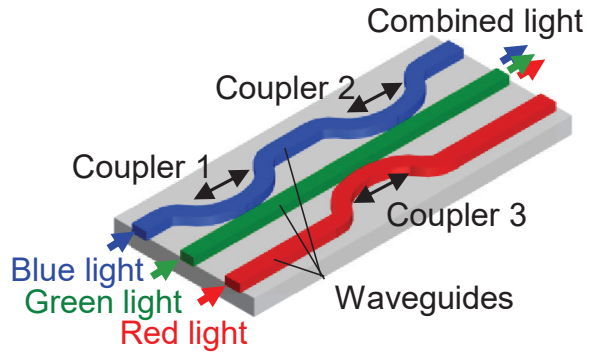

(a) Configuration.

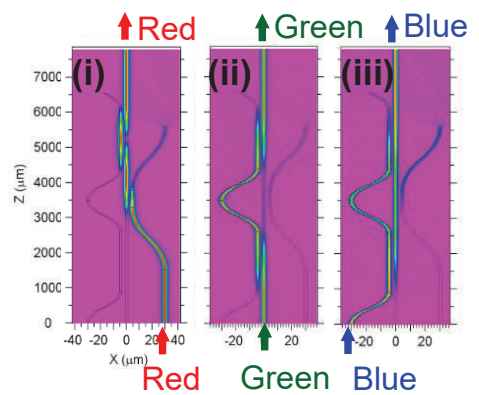

(b) RGB light transmission (Simulation).

(i) Red light, (ii) Green light, (iii) Blue light.

Fig. 1. Waveguide-type RGB multiplexer. directional couplers. The light transmission behaviour is shown in Fig. 1(b). Recently, we have obtained an extremely short coupling region, i.e., $2.9 \mathrm{~mm}$, leading to the realization of the compact laser beam projectors.

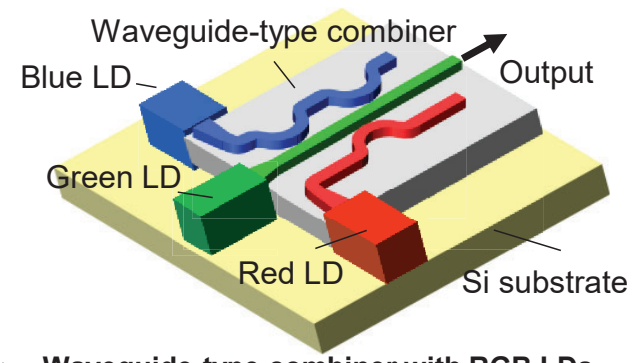

Waveguide-type combiner with RGB LDs

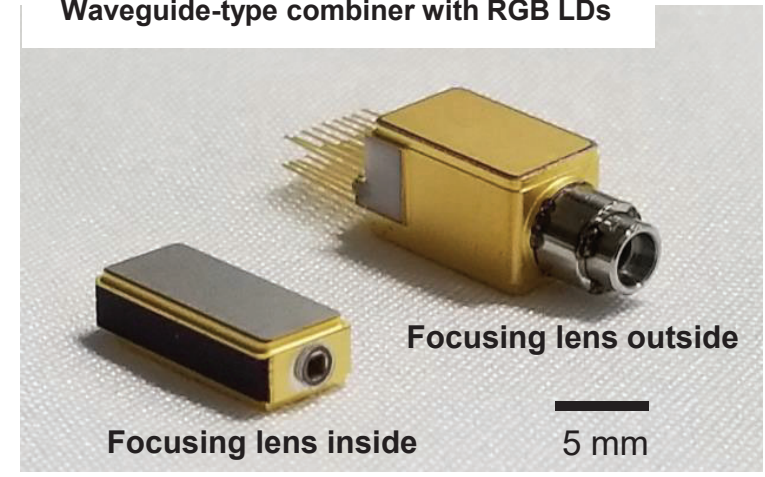

Fig. 2. Packaged RGB laser source modules.

\section{RGB laser sources}

The multiplexer integrated with RGB semiconductor lasers is schematically shown in Fig. 2 (inset). The measured multiplexing efficiency reaches as high as $97 \%$ ( $0.13 \mathrm{~dB}$ down). The multiplexer and RGB lasers are assembled on the same $\mathrm{Si}$ substrate. Each RGB output laser beam was perfectly aligned because of the inherent nature of the waveguide-type multiplexer.

Fig. 2 shows two types of packaged laser source modules. Their sizes are $10 \times 5 \times 5 \mathrm{~mm}$ for the lens-outside type and $8 \times 4 \times 3 \mathrm{~mm}$ for the lens-inside type. Since an achromatic lens is used, an excellent circular focused beam with no chromatic aberration was obtained. 


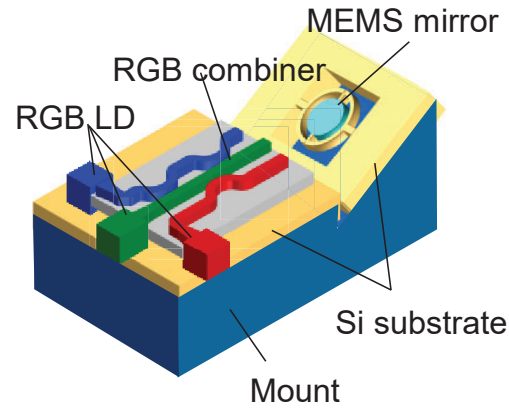

Fig. 3. Configuration of a RGB laser beam scanning module.

\section{RGB laser beam projectors}

We have assembled a RGB laser beam scanning module. Its configuration is shown in Fig. 3. A MEMS (Micro Electro Mechanical Systems) mirror for two-dimensional (2D) beam scanning is attached to the mount.

The fabricated RGB laser beam scanning projector is shown in Fig. 4. The optical scan angle is about 50 deg. for fast scan and its half angle for slow scan. The fast scan resonant frequency is about $30 \mathrm{kHz}$. Clear full-color video images with $1280 \times 720$ pixels have been obtained by using the above RGB laser beam scanning module.

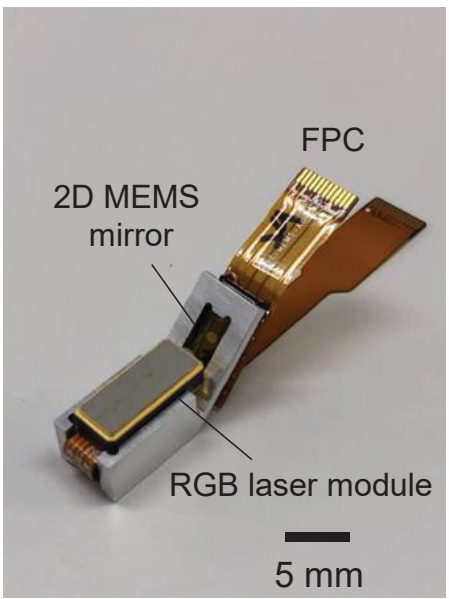

Fig. 4. Fabricated RGB laser beam scanning module.

\section{Candidate applications}

Our RGB laser sources and laser projection displays can be applied to industry/medical/healthcare/entertainment fields, as shown in Fig. 5. In particular, an automobile HUD (head up display) and entertainment MR (mixed reality) smart glasses are most important application candidates.

\section{Summary}

Compact full-color laser sources and laser beam scanning modules are demonstrated, which are based on waveguide-type RGB multiplexers. Since their output laser beams are perfectly aligned, compact imaging projectors showing clear images are constructed, which are applicable to a variety of imaging fields.

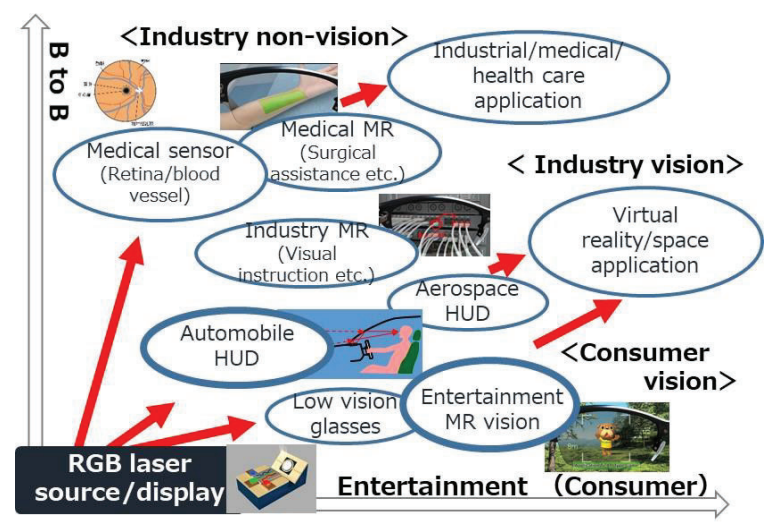

Fig. 5. Candidate applications of the RGB laser sources and RGB laser displays.

\section{Acknowledgment}

We wish to thank Profs. Yonezawa, Kosugi, Higuchi, Mrs. Okuno, Yoshimoto, Mses. Tokuda, Hirose of University of Fukui, and Mrs. Kawasaki, Kamei of SEIREN KST Corp. for their support throughout this work.

\section{References}

[1] B. C. Kress, "Optical Architectures for Augmented-, Virtual-, and Mixed-Reality Headsets", SPIE PRESS, Bellingham, Washington USA (2020).

[2] https://ethz.ch/en/the-eth-zurich/global/eth-global-newsevents/2019/09/eth-global-lecture-series-hololens-2unpacked.html, "ETH Global Lecture Series: HoloLens 2 Unpacked", 19.09.2019.

[3] https://en.retissa.biz/, "RETISSA", (2020).

[4] A. Nakao, R. Morimoto, Y. Kato, Y. Kakinoki, K. Ogawa, and T. Katsuyama, "Integrated waveguide-type red-greenblue beam combiners for compact projection-type displays," Opt. Commun. 330, 45-48 (2014).

[5] A. Nakao, K. Tsujino, S. Yokokawa, S. Tanaka, S. Hayashiguchi, and T. Katsuyama, "Waveguide-type Redgreen-blue Laser Beam Combiners Integrated with Semiconductor Lasers", The 22nd International Display Workshops (IDW '15), Otsu Prince Hotel, Otsu, Japan, PRJp1-5L (2015).

[6] A. Nakao, S. Hayashiguchi, S. Tanaka, S. Yokokawa, and T. Katsuyama, "Efficient laser beam coupling with a waveguide-type red-green-blue combiner", The 4th International Conference on Light-Emitting Devices and Their Industrial Applications (LEDIA' 16) , Yokohama, Japan, LED2-5 (2016).

[7] A. Nakao, S. Yamada, T. Katsuyama, O. Kawasaki, K. Iwabata, K. Horii, and A. Himeno, "A Compact Full-color Laser Beam Scanning Image Projector Based on a Waveguide-type RGB Combiner", The 27th International Display Workshops (IDW '20), Virtual Conference, PRJ3/FMC2-3 (2020). 
LDC2021 | Oral Presentation

AR, MR, VR, ...XR technologies 3 - xR concepts -

Tue. Apr 20, 2021 3:15 PM - 5:00 PM LDC (Online)

Click to the Online Room

[LDC-4-01 (Invited)] Introduction of xR use case at Toyota

OKoichi Kayano ${ }^{1}$ (1.Toyota Motor Corporation)

3:15 PM - 3:45 PM

[LDC-4-02 (Invited)] Introducing KDDI' s activities in XR and Spatial computing towards $5 \mathrm{G}$.

OKatsuhiro Kozuki ${ }^{1}$ (1.KDDI Corporation)

3:45 PM - 4:15 PM

[LDC-4-03] Deforming Aerial Image by Use of Deflection of Beam Splitter in See-Through AIRR

OKosuke Inoue $^{1}$, Masaki Yasugi ${ }^{1,2}$, Hirotsugu Yamamoto ${ }^{1,2}$ (1.Utsunomiya

University, 2.JST, ACCEL)

4:15 PM - 4:30 PM

[LDC-4-04 (Invited)] Why lasers are key to make smart and AR glasses the next big thing

OLucas Ginzinger $^{1}$ (1.Bosch Sensortec $\mathrm{GmbH}$ )

4:30 PM - 5:00 PM 
LDC2021 | Oral Presentation

$A R, M R, V R, \ldots X R$ technologies 3 - xR concepts -

Tue. Apr 20, 2021 3:15 PM - 5:00 PM LDC (Online)

\section{[LDC-4-01 (Invited)] Introduction of xR use case at Toyota}

OKoichi Kayano ${ }^{1}$ (1.Toyota Motor Corporation)

In general, there have been many examples of engineering using $x R$ technology. However, unfortunately, there are few examples of its use in the customer service field. Toyota is currently demonstrating the use of HoloLens 2 in dealerships. This presentation will explain the case study, results, and points to keep in mind. 


\title{
Introduction of $x R$ use case at Toyota
}

\author{
Koichi Kayano \\ ${ }^{1}$ Technical Service Division, Toyota Motor Corporation \\ 1-1-1 Yamabuki-cho, Tajimi, Gifu, 507-0802, Japan. \\ kayano@mail.toyota.co.jp
}

\begin{abstract}
In general, there have been many examples of engineering using $x R$ technology. However, unfortunately, there are few examples of its use in the customer service field. Toyota is currently demonstrating the use of HoloLens 2 in dealerships. This presentation will explain the case study, results, and points to keep in mind.
\end{abstract}

\section{Introduction}

For more than 20 years, Toyota has been utilizing the $\mathrm{CAD}$ data used in design for engineering in other businesses. In the past, this was mainly utilized using PC screens and large screens, but recently, with the emergence of inexpensive $\mathrm{xR}$ devices, the scope of utilization in real space has expanded. Here, we will explain the latest dealer utilization cases, achievements, and caution using mainly HoloLens.

\section{Background}

As cars become more complex, maintenance also becomes more difficult. There is also a shortage of experienced staff and it is becoming increasingly difficult to find young staff. We urgently need to reform how we do work.

\section{Issue and Aim}

The current manual is hard to read and understand.

Also, I can't see it right away.

It is necessary to learn various methods and how to find information, rather than learning the skills of maintenance work.

Therefore, we have focused on $\mathrm{xR}$ technology that can be used immediately in the field.

It can be used hands-free, and Microsoft's HoloLens was selected based on its relationship with existing Windows applications, and an innovative trial was carried out.

\section{Implementation}

We distributed HoloLens 2 to 57 GR Garage shops in Japan. Currently, we are demonstrating its effectiveness in actual stores. There are four main contents.
1) Electric Wire harness Diagram
2) Technical explanation
3) Remote Communication
4) Training and Guidance

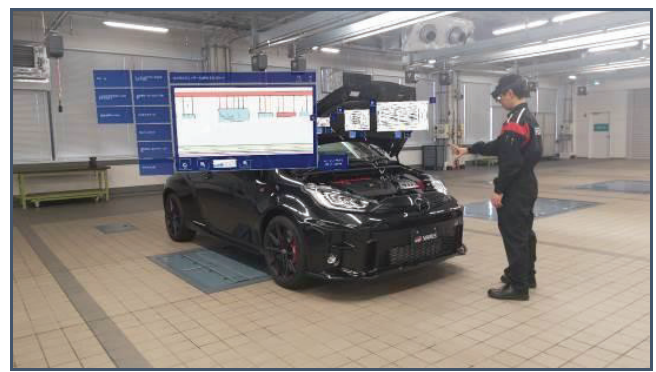

Fig.1 Sample of Electric Wire harness Diagram

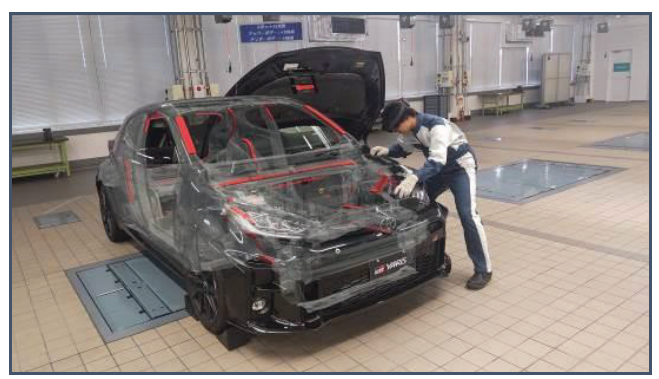

Fig.2 Sample of Technical Explanation

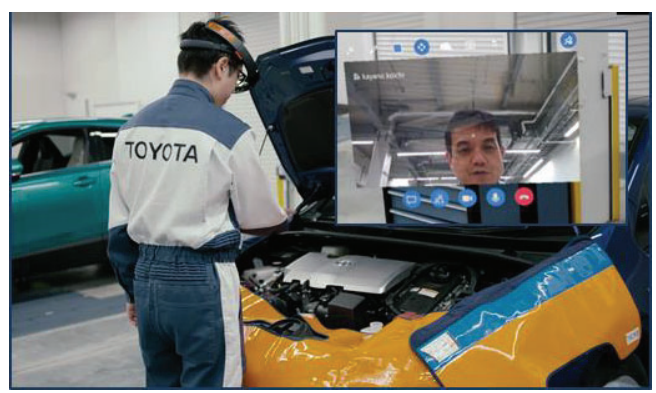

Fig.3 Sample of Remote Communication

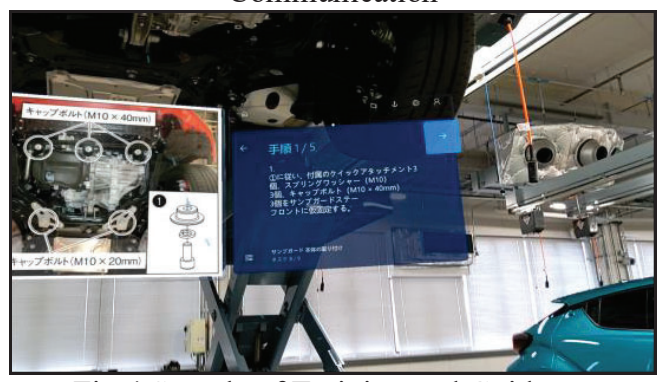

Fig.4 Sample of Training and Guidance 
We shall also present examples of proof of concept.

5) Work confirmation by AI

6) Remote workshop of vehicle by spatial recording

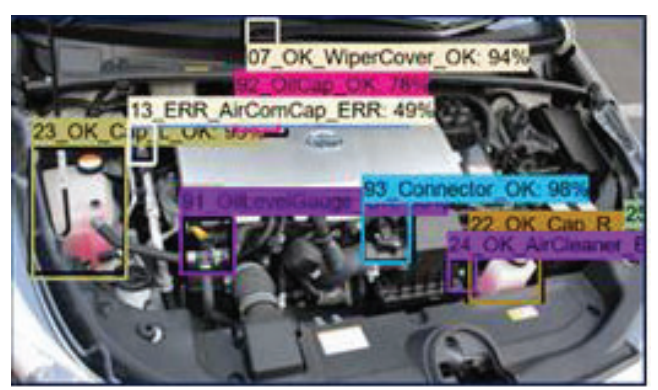

Fig.5 Sample of Work confirmation by AI

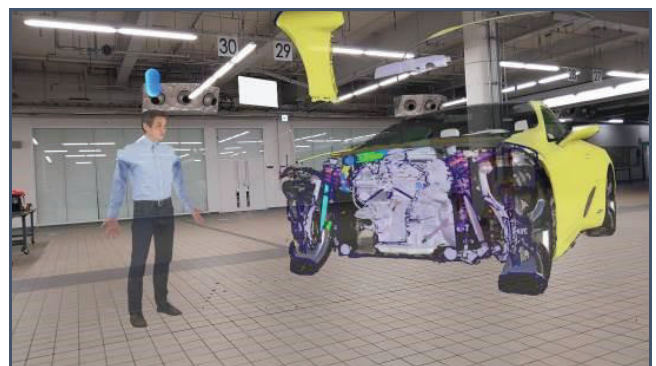

Fig.6 Remote workshop of vehicle by spatial recording

\section{Results}

It is an innovative initiative and is generally highly evaluated. Although it is an interim result, there are many comments to the effect that it wants to be used continuously. There are many improvements such as improved operability, and we will also enhance the target models. On the other hand, the need for remote support is increasing due to the corona virus, and there is a need to continue to enhance content such as training and guidance. The dealers are also expanding their own activities. We are promoting a futuristic work style image such as information dissemination through SNS and demonstration experience at job fairs for students. Through interviews with various media, it also contributes to improving the brand image of sales companies.

\section{Suggestions}

Unlike existing content, we have found that MR that display UI in space should consider the following. Typical examples are listed below.

- Small texts are remarkably difficult to see.

- The use of neutral colors or conservative contrast colors may help reduce fatigue.
- Holograms do not have a clickable feel, so they need to be responsive to actions, such as changing colors when they become active.

\section{Conclusion}

Through this initiative, we have realized that the on-site utilization of MR technology is sufficiently effective. We are also trying to use it in combination with spatial computing or AI, and we believe that the opportunities for utilization using MR devices will continue to expand in the future. 
LDC2021 | Oral Presentation

$A R, M R, V R, \ldots X R$ technologies 3 - xR concepts -

Tue. Apr 20, 2021 3:15 PM - 5:00 PM LDC (Online)

\section{[LDC-4-02 (Invited)] Introducing KDDI' s activities in XR and Spatial computing towards $5 \mathrm{G}$. \\ OKatsuhiro Kozuki ${ }^{1}$ (1.KDDI Corporation)}

The presentation will explain about KDDI' s recent activities in VR, AR and MR(collectively XR) enhanced by its $5 \mathrm{G}$ system, mobile edge computing and AR cloud technologies with $5 \mathrm{G}$ smartphone and tethered AR glasses products. Introducing from PoC level promotional events to commercially available products and services on $5 \mathrm{G}$ networks. 


\title{
Introducing KDDI's Activities in XR and Spatial Computing Towards 5G
}

\author{
Katsuhiro Kozuki, KDDI Corporation, \\ Iidabashi First Tower, 2-6-1 Koraku, Bunkyo-ku, Tokyo Japan 112-0004 \\ +81 80-5076-9968, kozuki@kddi.com
}

\begin{abstract}
This will explain about KDDI's recent activities in VR, AR and MR(collectively XR) enhanced by its $5 G$ system, mobile edge computing and AR cloud technologies with $5 G$ smartphone and tethered AR glasses products. Introducing from PoC level promotional events to commercially available products and services on $5 G$ networks.
\end{abstract}

\section{Introduction}

The latest XR technology enhanced by $5 \mathrm{G}$ system, which we believe is the next mobile computing platform and will potentially transform how the world connects, computes, and communicates. Last year, KDDI has launched a commercial $5 \mathrm{G}$ service in Japan and started providing its $5 \mathrm{G}$ products and services to both of enterprise clients and consumers, enabling more extended and immersive user experiences compare to the conventional 4G/LTE based ones.

Our current products include $5 \mathrm{G}$ smartphones and $\mathrm{XR}$ devices such as AR glasses tethered to $5 \mathrm{G}$ smartphones with an USB-C cable.

We also set up our Digital Twin concept based services like "Virtual Shibuya (Virtual City)" in VR and "AR Cloud" using VPS(Visual Positioning Service) with partners, VPS uses computer vision technology to detect the exact location mapping information, and reconstruct intended objects and put them in the real world based on a geometry data on the AR cloud server.

The situation around XR business has been changed dramatically after the spread of COVID-19 and there are great expectations to fill the space of emptiness in social activities in the real world.

\section{KDDI's activities in XR and Spatial Computing on} 5G network

$5 \mathrm{G}$ network service expansion is still undergoing. Some $5 \mathrm{G}$ peripheral devices include AR glasses with their applications are ready to use but not enough yet. The Digital Twin services such as VR-based Virtual City (ex, Virtual Shibuya) and AR-based AR Cloud have recently launched commercially from Tokyo region.

Low-latency and high-resolution video streaming system via $5 \mathrm{G}$ system enables to reconstruct a higher quality CGI (Computer-Generated Imagery) or even a Digital Human on the devices.

Telepresence, spatial computing service with a partner is in a trial phase.

There are some changes in our forecast due to the COVID-19 concerns, but we also have expectations in this space.

\subsection{G development status and plan}

The 5G network coverage is not enough to experience its service nation widely yet. However, especially adding or allocating the existing $4 \mathrm{G} / \mathrm{LTE}$ radio bands to $5 \mathrm{G}$ system will help expanding the $5 \mathrm{G}$ coverage efficiently from a metropolitan area to a residential one, and will popularize $5 \mathrm{G}$ among mobile subscribers with its optimized services even if the $5 \mathrm{G}$ service still has some limitations in terms of the network speed.

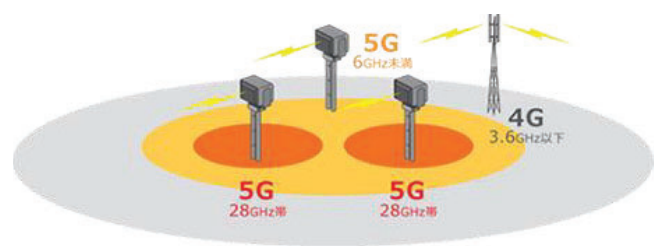

Fig. 1. Overview of $4 \mathrm{G}$ and $5 \mathrm{G}$ service coverage.

\section{$2.25 \mathrm{G}$ devices}

We have started providing $5 \mathrm{G}$ smartphones from last year and the all of our smartphones are $5 \mathrm{G}$ enabled already. To extend user experiences with $5 \mathrm{G}$ network service, we are trying to offer additional products such as NrealLight (AR glasses, manufacture Nreal Ltd.) to be tethered to $5 \mathrm{G}$ smartphones. This also means we would expect our customers to subscribe a higher data plan to experience immersive XR services more from the mobile operator's business point of view. The most of existing $\mathrm{XR}$ devices are kinds of stand-alone devices and works only with Wi-Fi connectivity; they will not generate additional mobile network usage by themselves and to be used within doors mainly. This is one of the reasons for us to deal with $5 \mathrm{G}$ smartphone tethered-type devices.

The AR glasses allow user to launch and use multiple existing mobile applications such as video streaming services or SNSs concurrently in their sights and locate those screens virtually in MR (Mixed-Reality) mode freely, and this will increase the data traffic as well. 


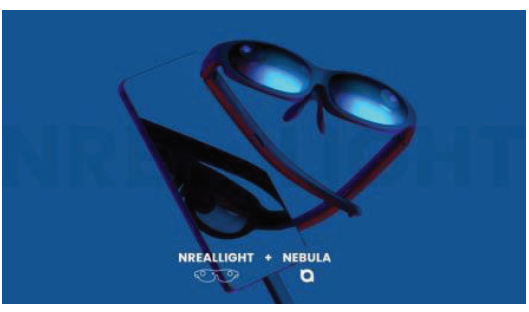

Fig. 2. Product image of NrealLight(AR glasses) with $5 \mathrm{G}$ smartphone.

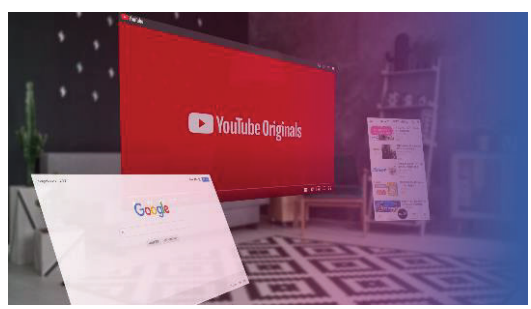

Fig. 3. Image view using NrealLight (AR glasses) in Mixed Realty mode.

2.3 Digital Twin and 5G MEC

Digital twin services such as "Virtual City (Shibuya)" and AR Cloud have started running on the cloud. We have done some additional service trials on AR Cloud with a $5 \mathrm{G}$ MEC capability to reduce the latency of sensing a real world through a camera equipped on a $5 \mathrm{G}$ smartphone or AR glasses for the better experience. The GPU performance on the MEC helps rendering CGI or digital human to be more photorealistic, and they can be streamed to $5 \mathrm{G}$ devices as visual image data including stereoscopic (3D) reconstructions.

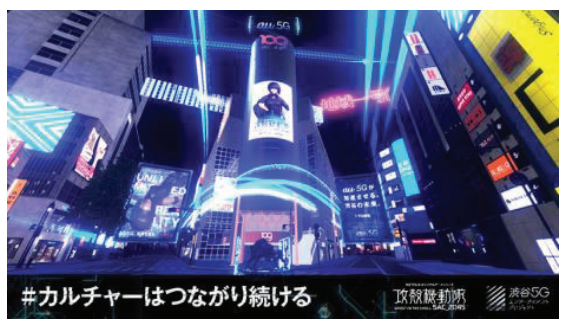

Fig. 4. Virtual Shibuya (virtual city) in VR.
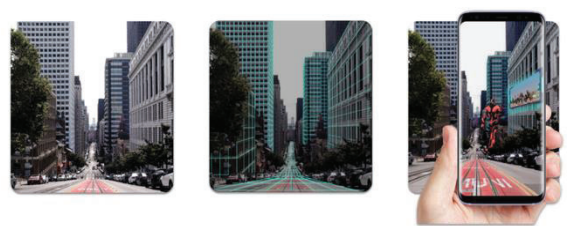

Fig. 5. AR Cloud using VPS technology.

2.4 Telepresence-spatial computing

There is a new demand for $3 \mathrm{D} /$ holographic collaboration platform service with XR devices. KDDI and our strategic partner Spatial Systems Inc. will provide cloud based 3D communication functionality using NrealLight AR glasses connected to $5 \mathrm{G}$ smartphones.

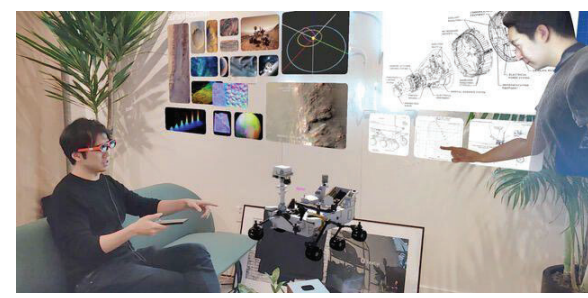

Fig. 6. Holographic collaboration platform "Spatial" [1].

2.5. Marketing review of changes after the spread of COVID-19. We have seen a huge increase in demand for XR business after the spread of COVID-19 from last year. The most of planned physical events have been postponed or canceled. Alternatively, forced to be done them online. There are some actual cases like setting up a virtual shop online with a digital human, and there is a story behind them from both of technologies and marketing points of view.

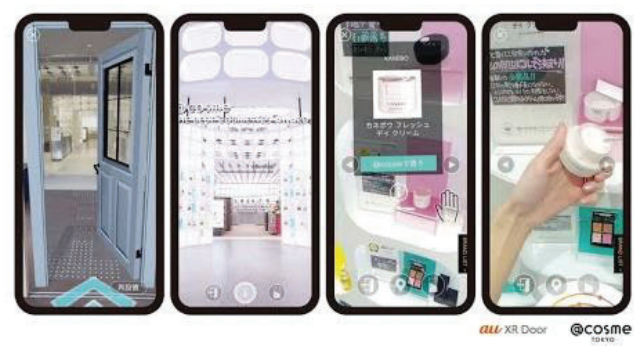

Fig. 7.@cosme TOKYO (virtual shop).

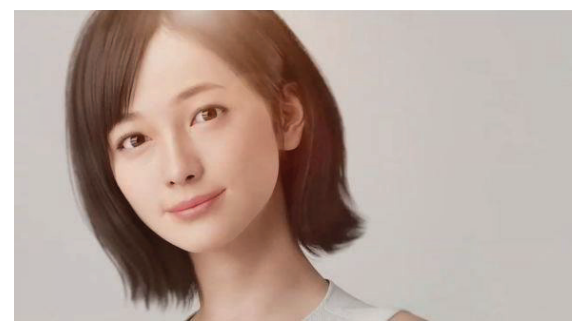

Fig. 8. KDDI original digital human (computergenerated human).

\section{Conclusion}

$\mathrm{XR}$ related industry market size will expand more as enhanced 5G system (5G-SA) and optimized application services go live. The required hardware technologies including displays and optics for head-worn devices expected to be more important in the next few years.

\section{Refereces}

[1] "Spatial" demo screen image provided from Spatial Systems Inc. 
LDC2021 | Oral Presentation

$A R, M R, V R, \ldots X R$ technologies 3 - xR concepts -

Tue. Apr 20, 2021 3:15 PM - 5:00 PM LDC (Online)

\section{[LDC-4-03] Deforming Aerial Image by Use of Deflection of Beam Splitter in See-Through AIRR}

OKosuke Inoue ${ }^{1}$, Masaki Yasugi ${ }^{1,2}$, Hirotsugu Yamamoto ${ }^{1,2}$ (1.Utsunomiya University, 2.JST, ACCEL)

This paper proposes a novel aerial display optics to display a three-dimensional aerial image by intentionally creating irregular deflection. Irregular deflection is occurred by pulling the acrylic plate forward with a piano wire and partially raising it. We succeeded in forming aerial images that appear differently depending on the viewpoint. 


\title{
Deforming Aerial Image by Use of Deflection of Beam Splitter in See-Through AIRR
}

\author{
Kosuke Inoue $^{1}$, Masaki Yasugi ${ }^{1,2}$, Nao Ninomiya ${ }^{1}$, Hirotsugu Yamamoto ${ }^{1,2}$ \\ ${ }^{1}$ Utsunomiya University, 7-1-2 Yoto, Utsunomiya City, Tochigi, 321-0904 Japan, \\ +81-28-689-7074, E-mail: hirotsugu@yamamotolab.science. \\ ${ }^{2}$ JST, ACCEL, 7-1-2 Yoto, Utsunomiya City, Tochigi, 321-0904 Japan
}

\begin{abstract}
This paper proposes a novel aerial display optics to display a three-dimensional aerial image by intentionally creating irregular deflection. Irregular deflection is occurred by pulling the acrylic plate forward with a piano wire and partially raising it. We succeeded in forming aerial images that appear differently depending on the viewpoint.
\end{abstract}

\section{Introduction}

An aerial image by retro-reflection (AIRR) is one of methods to form aerial floating image and has the advantage of being easy to realize larger aerial image by arranging the retro-reflective sheet in tiles [1]. We found that the device using acrylic plate as a beam splitter realized a fine aerial image, however the device had the problem that the aspect ratio of aerial image was different from the one of original picture on the light source $[2,3]$. This is because the acrylic plate slantly installed by 45 degrees was deformed by its own weight.

Although AIRR features a wide viewing angle, a simple aerial image formation results in a flat image even when the viewpoint is changed. Our previous optical system was developed in order to reduce aberrations in the aerial image by using piano wire to support a deflected acrylic plate. However, the deformability of the beam splitter is an advantage of using an acrylic plate, which leads to a breakthrough from flat aerial images.

The purpose of this research is to display threedimensional aerial images by intentionally deflecting the beam splitter irregularly using a piano wire.

\section{Principle}

\subsection{AIRR}

AIRR is composed of three components: a light source, retro-reflector, and a beam splitter. Fig.1(a) shows the principle of AIRR. Light emitted from the light source is split into reflected light and transmitted light by the beam splitter. The reflected light is retro-reflected by the retro-reflector and then returns to the beam splitter. This retro-reflected light is also split by the beam splitter. The transmitted light converges to the plane symmetric position of the light source with respect to the beam splitter.

\subsection{See-Through AIRR}

See-through AIRR is one of the variations in optical design of AIRR. Fig.1(b) shows the principle of seethrough AIRR. The advantage of the aerial image display using AIRR is that the viewing angle is as wide as 120 degrees. However, the basic structure of AIRR is specialized for frontal observation. In the see-through
AIRR, the retroreflective sheet, which is on the straight line of the image formation position in the AIRR, is placed at the top of the system. The light emitted from the light source is divided into reflected light and transmitted light by a beam splitter. The transmitted light is retroreflected by the retroreflective sheet, and then returns to the beam splitter. The reflected light is reflected again by the beam splitter to form an aerial image in front of the device. On the other hand, the light emitted from the light source and reflected by the beam splitter forms an aerial image as an imaginary image on the other side of the device. The position of the real image observed by the user from the front side and the imaginary image observed from the other side are almost the same, so the user can observe the operation performed on the aerial image from the other side.

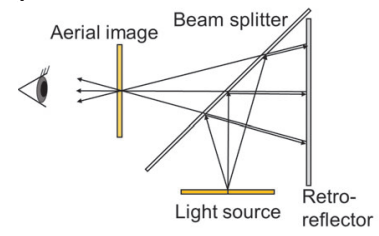

(a)

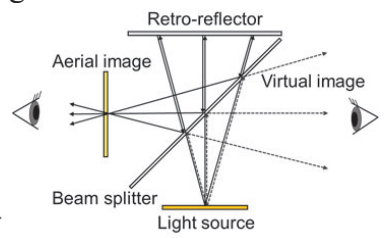

(b)
Fig.1. Principle of (a) AIRR and (b) see-through AIRR.

\section{Aberration caused by a curved beam splitter}

A large aerial display system is shown in Fig. 2(a). When the beam splitter is slantly installed by 45 degrees, the light emitted from the light source follows the optical path shown in blue and forms an aerial image at the position shown by the dotted line. However, the large acrylic plate bends under its own weight. Therefore, the retro-reflected light follows the light path shown in orange. Thus, a distorted aerial image is formed at the position shown by the solid line.

In this study, the aberration of the aerial image is reduced by using piano wire to support the acrylic plate from behind. The degree of aberration reduction was evaluated by displaying an image of squares regularly arranged on an LED panel as a light source, photographing the aerial image, and measuring the grid [4]. We examined the effect of the diameter and arrangement of wire on the distortion of aerial image. The comparison results are shown in Fig. 2(b). In 
Condition A, a $5 \mathrm{~mm}$ acrylic plate was placed on the apparatus without using piano wires. In Condition $\mathrm{B}$, a $2 \mathrm{~mm}$ acrylic plate was supported by four $0.3 \mathrm{~mm}$ piano wires, and in Condition $\mathrm{C}$, the number of piano wires was increased to 10 . In Condition $\mathrm{D}$, a $2 \mathrm{~mm}$ acrylic plate was supported by four $0.8 \mathrm{~mm}$ piano wires. In Condition E, a $1 \mathrm{~mm}$ acrylic plate was supported by four $0.8 \mathrm{~mm}$ piano wires. The comparison results show that the aberration was reduced as the number of supported piano wires was increased. Furthermore, the thinner the acrylic plate, the more easily it was deflected.

Consequently, the aberration of the aerial image increases with the curvature of the beam splitter. In other words, a curved beam splitter can form a curved aerial image by use of a flat light-source display.

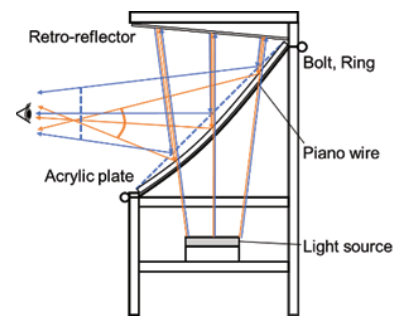

(a)

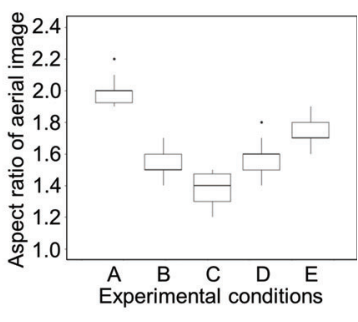

(b)
Fig.2. Conventional optical system and experimental results. (a) Aberration of an aerial image due to deflection of an acrylic plate, (b) Comparison of aberrations of aerial images for different conditions.

\section{Optical system for deformed aerial image}

Our proposed optical system is shown in Fig.3. As in Fig.2(a), an acrylic plate $(1000 \mathrm{~mm} \times 1280 \mathrm{~mm})$ is used as the beam splitter and supported by four piano wires with a diameter of $0.8 \mathrm{~mm}$. When the beam splitter is placed at 45 degrees, the light emitted from the light source forms an aerial image at the dotted gray line, which is symmetrical to the beam splitter. However, the large acrylic plate bends under its own weight, so the aerial image is formed at the position of the solid orange line. When the acrylic plate is pulled forward with a piano wire and partially raised, irregular deflection occurs, and the image is formed at the position shown by the solid blue line.

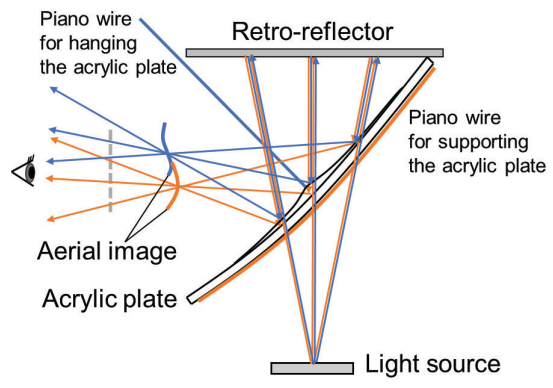

Fig. 3. Deforming the aerial image formed with AIRR by use of a curved acrylic plate.

\section{Experiment}

An image of a fireball was displayed on an LED panel as a light source. Fig. 4 shows the observation result of the acrylic plate supported only from behind. Fig. 5 shows the observation result of the acrylic plate suspended from the front. By shifting the position of the aerial image, the image was perceived as three-dimensional, while a simple aerial image gave a flat impression. This is an advantage of using acrylic plate, which is easy to change its shape, for the beam splitter. It is possible to change the appearance of the aerial image depending on how it is pulled.

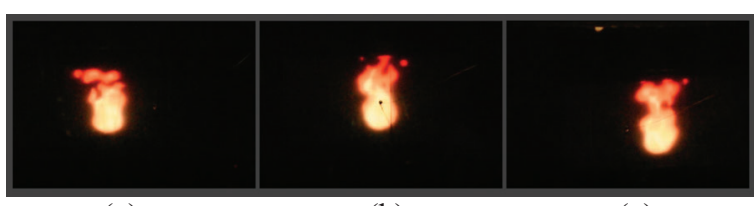

(a)

(b)

(c)

Fig. 4. Aerial image formed by use of the acrylic plate supported only from behind, viewed (a) from the right, (b) in front, and (c) from the left.

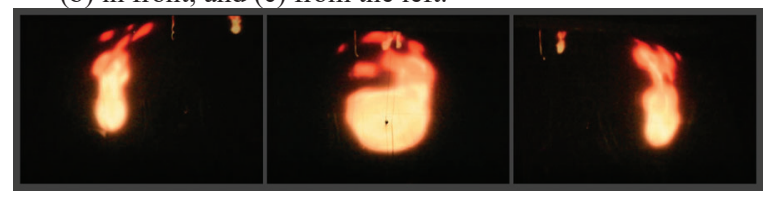

(a)

(b)

(c)

Fig. 5. Aerial image using a piano wire for hanging the acrylic plate, viewed (a) from the right, (b) in front, and (c) from the left.

\section{Conclusion}

We have succeeded in deforming aerial images that appear differently depending on the viewpoint position by utilizing the deflection of the beam splitter. Irregular deflection was occurred by pulling the acrylic plate forward with a piano wire and partially raising it. The three-dimensional aerial image was formed by using an easily deformable acrylic plate. One of the prospective applications of this work is to show a virtual fire flame in an electric heater.

A part of this work was supported by JST/ACCEL (grant no. JPMJAC1601) and JSPS KAKENHI (19H04155, 20H05702).

\section{References}

[1] H. Yamamoto, Y. Tomiyama, and S. Suyama, "Floating aerial LED signage based on aerial imaging by retro-reflection (AIRR)," Opt. Express 22, 2691926924 (2014).

[2] M. Yasugi, H. Yamamoto, and Y. Takeda, "Immersive aerial interface showing transparent floating screen between users and audience," Proc. SPIE 11402, 1140200 (2020).

[3] Y. Seki, R. Kujime, and H. Yamamoto, "Forming an Enlarged Aerial Image by Use of a Curved Beam Splitter in AIRR," Proc. IMID 2018 DIGEST, 476 (2018)

[4] K. Inoue, M. Yasugi, and H. Yamamoto, "Reducing Aberration of Aerial Image by Use of Supporting Wire in Large Aerial Display with AIRR," Proc. IDW'20, pp. 715-718 (2020). 
LDC2021 | Oral Presentation

$A R, M R, V R, \ldots X R$ technologies 3 - xR concepts -

Tue. Apr 20, 2021 3:15 PM - 5:00 PM LDC (Online)

\section{[LDC-4-04 (Invited)] Why lasers are key to make smart and AR glasses the next big thing \\ OLucas Ginzinger ${ }^{1} \quad$ (1.Bosch Sensortec $\mathrm{GmbH}$ )}

The world has been waiting for the big bang in consumer smart glasses not only since Google Glass. Besides use case readiness, the tech maturity is playing a major role in the delayed market of smart/AR glasses for consumers. In his presentation, Lucas Ginzinger, Head of Product Area Optics, talks on the major levers to overcome the hurdles for a successful market entry - the role of laser beam scanning projectors as the key enablers to high volume smart and AR glasses. 
The 10th Laser Display and Lighting Conference 2021 (LDC 2021), Apr. 19 - Apr. 22, 2021

(C) OPTICS \& PHOTONICS International 2021

\title{
Why lasers are key to make smart and AR glasses the next big thing
}

\author{
Lucas Ginzinger
}

Bosch Sensortec GmbH, Gerhard-Kindler-Straße 9, 72770 Reutlingen, GERMANY, +49 7121 35-36705

Lucas.Ginzinger@bosch-sensortec.com

\begin{abstract}
The world has been waiting for the big bang in consumer smart glasses not only since Google Glass. Besides use case readiness, the tech maturity is playing a major role in the delayed market of smart/AR glasses for consumers. In his presentation, Lucas Ginzinger, Head of Product Area Optics, talks on the major levers to overcome the hurdles for a successful market entry - the role of laser beam scanning projectors as the key enablers to high volume smart and AR glasses.
\end{abstract}

Proc. of SPIE Vol. 11851 1185101-53 
LDC2021 | Oral Presentation

Imaging / Lighting

Wed. Apr 21, 2021 10:00 AM - 11:45 AM LDC (Online)

Click to the Online Room

[LDC-5-01 (Invited)] Laser TV progress with visible laser improvement

OXianrong Liu1 (1.Hisense) NOSHOW

10:00 AM - 10:30 AM

[LDC-5-02 (Invited)] Large-scale full-parallax full-color computer-generated

holograms reconstructed by laser/LED lighting

OKyoji Matsushima1 (1.Kansai University)

10:30 AM - 11:00 AM

[LDC-5-03] Improvement of Color Rendering Index of BGYR Laser illuminats

OYoshio Manabe1, Masato Ishino1, Hiroshi Fuji1, Akira Takamori1, Junichi

Kinoshita1, Kana Fujioka1, Kazuhisa Yamamoto1 (1.Institute of Laser Engineering,

Osaka University)

11:00 AM - 11:15 AM

Color Shift Behavior at Image Pattern Edges of Raster-scan RGB

[LDC-5-04]

Mobile Laser Projectors

OJunichi Kinoshita1, Akira Takamori1, Kazuhisa Yamamoto1, Kazuo Kuroda2, Koji

Suzukiз (1.Osaka Universitry, 2.Utsunomiya University, 3.OXIDE Corporation)

11:15 AM - 11:30 AM

[LDC-5-05]

Ultra high resolution and VAC-free N3D technology and its applications

OChin-Yung Hsieh1, Hao-Yu Liu1, Ruey-Jer Weng1, Wei-Yi Lu1, Naoki Sumi2

(1.Innolux Corporation, 2.Innolux Japan K.K.)

11:30 AM - 11:45 AM 
LDC2021 | Oral Presentation

Imaging / Lighting

Wed. Apr 21, 2021 10:00 AM - 11:45 AM LDC (Online)

[LDC-5-01 (Invited)] Laser TV progress with visible laser improvement OXianrong Liu ${ }^{1}$ (1.Hisense)

NO SHOW 
LDC2021 | Oral Presentation

Imaging / Lighting

Wed. Apr 21, 2021 10:00 AM - 11:45 AM LDC (Online)

[LDC-5-02 (Invited)] Large-scale full-parallax full-color computergenerated holograms reconstructed by laser/LED lighting

OKyoji Matsushima ${ }^{1} \quad$ (1.Kansai University)

Recently, full-color computer-generated holograms (CGH) are created by several techniques. These CGHs can be reconstructed using an LED or RGB laser light source. The creation and lighting techniques are introduced for promoting development of light sources for computer holography. 


\title{
Large-Scale Full-Parallax Full-Color Computer-Generated Holograms Reconstructed by Laser/LED Lighting
}

\author{
Kyoji Matsushima ${ }^{1}$ \\ ${ }^{1}$ Department of Electrical and Electronic Engineering, Kansai University, \\ Yamate-cho 3-3-35, Suita, Osaka 564-8680, Japan, +81-6-6368-0933, matsu@kansai-u.ac.jp
}

\begin{abstract}
Two techniques are introduced to create full-color computer-generated holograms that have very large-scale such as tens of billion pixels and reconstruct a very impressive deep $3 D$ scene. In the techniques, white LEDs and RGB laser are used as the illumination light source. Optical reconstruction of these full-color CGHs are demonstrated in this paper.
\end{abstract}

\section{Introduction}

Computer holography, the technique to create holographic 3D images using a computer, has been developed over the last decade [1]. The created holograms, called computer-generated holograms (CGH), can reconstruct $3 \mathrm{D}$ images of non-physical objects unlike traditional optical holography. Unfortunately, creating high-quality CGHs had been impossible for a long time because of the gigantic space-band product required for realizing a given large viewing angle and screen size. We developed several techniques that make it possible to calculate and fabricate large-scale CGHs, called high-definition CGHs, which are composed of billions or sometimes tens or hundreds of billion pixels (Chap. 1 in [1]). The large-scale CGHs reconstruct spatial 3D images of a deep scene with natural motion parallax in full-parallax. The 3D images, in fact, are being comparable to those in traditional optical holography. As a result, the CGHs give great impact of strong sensation of depth to viewers.

However, it was very difficult to reconstruct fullcolor images by these high-definition CGHs because the printed CGHs are a type of thin hologram. The 3D images reconstructed under a white-light illumination cause many smears because of chromatic aberration. Recently, two methods: CGHs with RGB color filters [2, 3] and stacked CGVHs (computer-generated volume hologram) [4] were invented for reconstructing full color images from high-definition CGHs. These full-color CGHs are reconstructed using a white LED or RGB laser light sources.

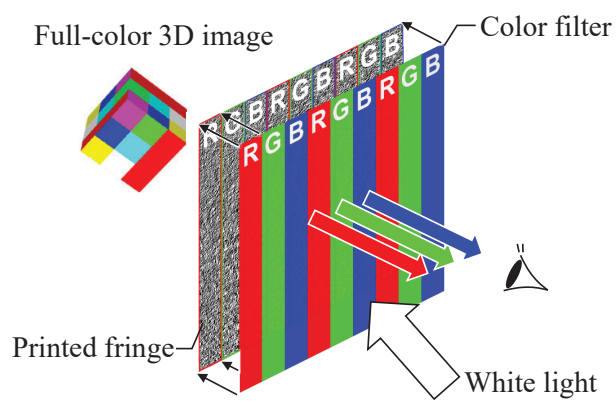

Fig. 1. Full-color reconstruction of high-definition CGHs using RGB color filters.

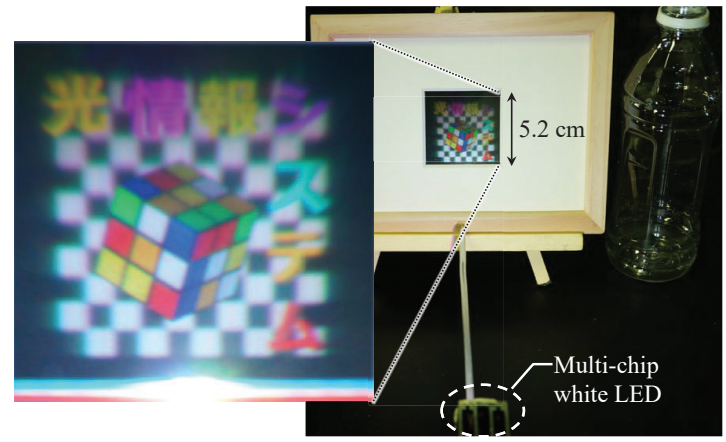

Fig. 2. Full-color reconstruction of a high-definition CGH using RGB color filters [2]. Video link, https://doi.org/10.1364/OE.25.002016.v001

\section{CGH with RGB color filters}

In this technique, RGB color filters that are very similar to those used in LCD panels are attached to the largescale fringe pattern, and the fringe pattern behind the filters is generated at appropriate wavelengths corresponding to the RGB colors, as shown in Fig. 1.

\subsection{Illumination light sources}

CGHs with RGB color filters can be reconstructed by both LEDs and RGB lasers. When using an LED, because the bandwidth of the RGB color filters is commonly not so narrow, a multi-chip type white LED should be used instead of a single-chip type with phosphor to reconstruct sharp 3D images. The problem of the bandwidth is properly avoided by use of an RGB laser source.

\subsection{Optical reconstruction}

Optical reconstruction using a multi-chip white LED is shown in Fig. 2. The 3D image is blurred a little by the wideband property of the illumination. Coherent illumination using an RGB laser makes the reconstructed image much sharper as shown in Fig. 3. Here, an ultrashort focus projector made by SONY ${ }^{\mathrm{TM}}$, where RGB laser diodes (LD) are installed, is used for the light source, as in (d). Note that the projector throws just a plane white image in this case. We can also use discrete RGB-LD components for the illumination, as in (e). 


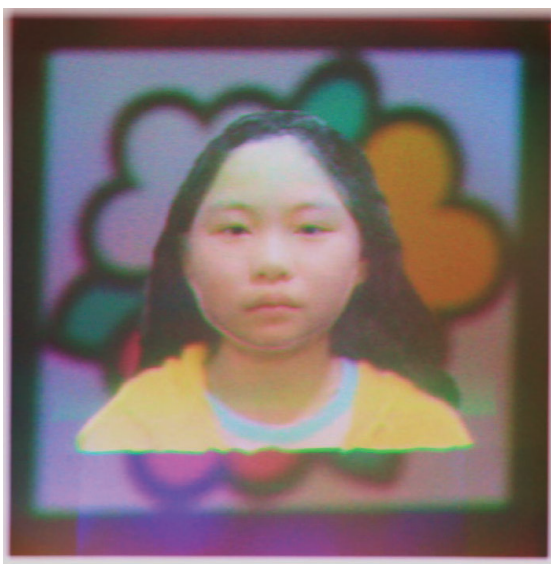

(a)

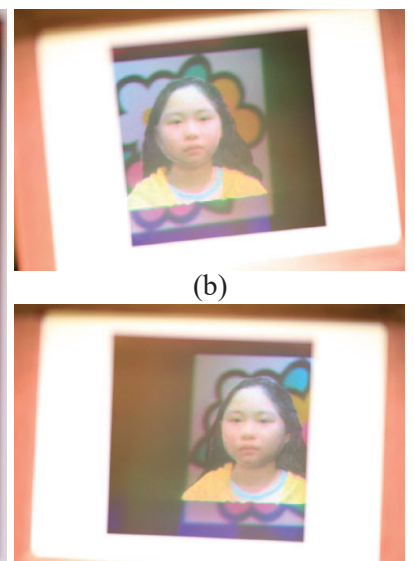

(c)

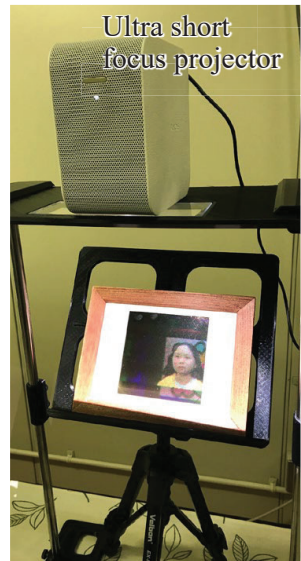

(d)

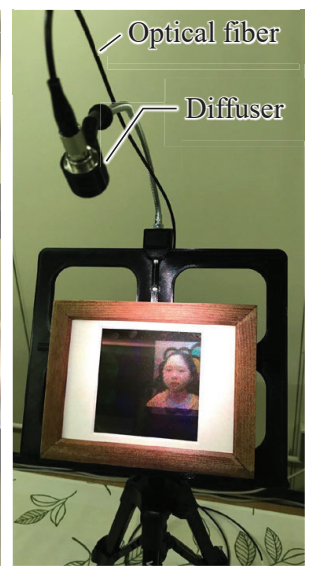

(e)

Fig. 3. A CGH with RGB color filters reconstructed using RGB lasers. A close-up image (a), and images from (b) left and (c) right viewpoints (Sect. 15.6.4 in [1]); Illumination using (d) a laser projector and (e) RGB laser.

Video link, https://youtu.be/DmC93CRdhFQ

\section{Stacked CGVH}

Another way to reconstruct full-color 3D images from a high-definition $\mathrm{CGH}$ is stacking computer-generated volume holograms (CGVH) [4]. A volume hologram, sometimes called a "thick" hologram, is a different type of hologram. The volume hologram cannot be created by printing the fringe pattern because the fringe has a $3 \mathrm{D}$ structure in the recording material such as photopolymer. We can fabricate CGVHs by transferring the original $\mathrm{CGH}$, printed using laser lithography (Sect. 15.3 in [1]), to photopolymer using the technique of contact-copy.

A volume hologram has strong wavelength selectivity based on Bragg reflection, and can be reconstructed by reflection illumination only at wavelength used in the contact copy. Thus, we can reconstruct full-color images by fabricating three CGVHs from three original printed CGHs and stacking the CGVHs, as shown in Fig. 4. The hologram reconstructs very fine $3 \mathrm{D}$ images shown in Fig. 5 using a single-chip white LED, which has a broadband property, because of the excellent wavelength selectivity of the CGVHs.

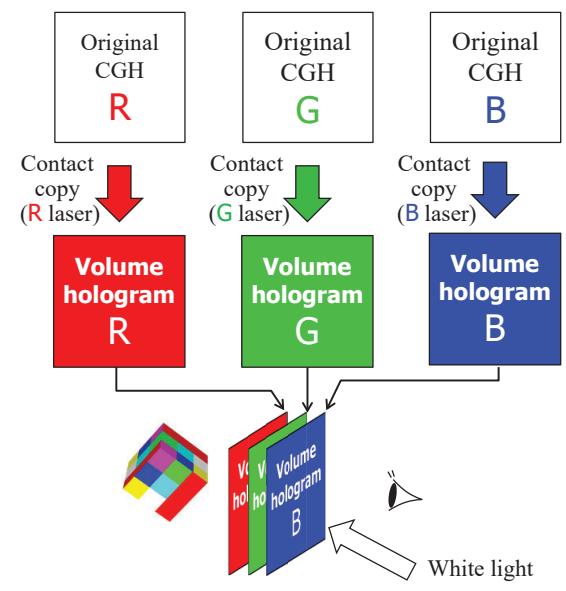

Fig. 4. The principle of stacked CGVHs.

\section{Conclusion}

Full-color reconstruction of large-scale CGHs is achieved by two methods using a white LED and RGB laser light source.

\section{Acknowledgment}

This work was partially supported by Japan Society for the Promotion of Science (JSPS) KAKENHI $18 H 03349$.

\section{References}

[1] K. Matsushima, Introduction to Computer Holography (Springer, 2020).

[2] Y. Tsuchiyama and K. Matsushima, "Full-color largescaled computer-generated holograms using RGB color filters," Opt. Express 25, 2016-2030 (2017).

[3] K. Matsushima and N. Sonobe, "Full-color digitized holography for large-scale holographic 3D imaging of physical and nonphysical objects," Appl. Opt. 57, A150 A156 (2018).

[4] O. Kunieda and K. Matsushima, "High-quality full-parallax full-color three-dimensional image reconstructed by stacking large-scale computer-generated volume holograms," App. Opt. 58, G104-G111(2019).

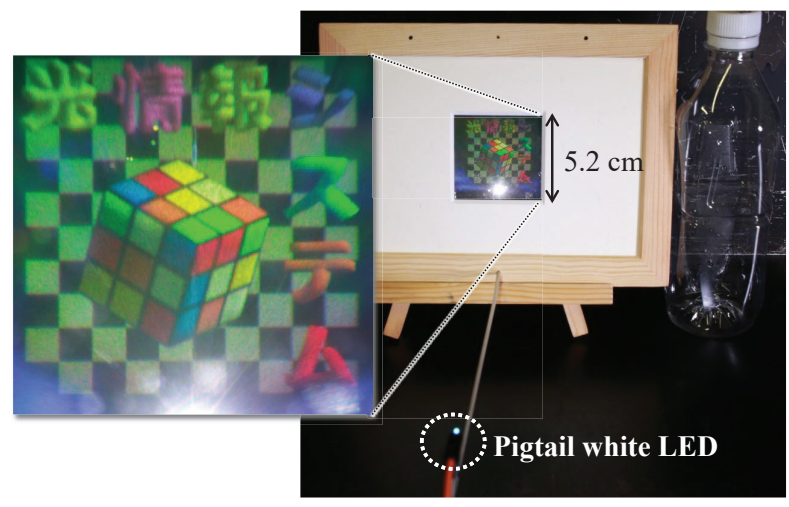

Fig. 5. Full-color reconstruction of the stacked CGVH using a broadband pigtail white LED [4]. Video link, https://doi.org/10.6084/m9.figshare.8870177.v1 
LDC2021 | Oral Presentation

Imaging / Lighting

Wed. Apr 21, 2021 10:00 AM - 11:45 AM LDC (Online)

\section{[LDC-5-03] Improvement of Color Rendering Index of BGYR Laser} illuminats

OYoshio Manabe ${ }^{1}$, Masato Ishino ${ }^{1}$, Hiroshi Fuji ${ }^{1}$, Akira Takamori ${ }^{1}$, Junichi Kinoshita ${ }^{1}$, Kana Fujioka ${ }^{1}$, Kazuhisa Yamamoto $^{1}$ (1.Institute of Laser Engineering, Osaka University)

We have attempted to improve Color Rendering Index of Blue-Green-Yellow-Red four-color Laser illuminants. As a result, average color rendering index Ra and special CRI( R9) as high as 80 around at $5000 \mathrm{~K}$ were obtained by optimizing the BGYR wavelengths and their laser power densities. 


\title{
Improvement of Color Rendering Index of BGYR Laser illuminats
}

\author{
Yoshio Manabe ${ }^{1}$, Masato Ishino, Hiroshi Fuji ${ }^{1}$, Akira Takamori ${ }^{1}$, Junichi Kinoshita ${ }^{1}$, Kana Fujioka ${ }^{1}$ and Kazuhisa Yamamoto ${ }^{1}$ \\ ${ }^{1}$ Institute of Laser Engineering, Osaka University 2-6 Yamadaoka, Suita, Osaka, 565-0871, Japan \\ Phone+81-6-6105-5299, email manabe-y@ile.osaka-u.ac.jp
}

\begin{abstract}
We have attempted to improve Color Rendering Index of Blue-Green-Yellow-Red four-color Laser illuminants. As a result, average color rendering index $R_{a}$ and special $C R I\left(R_{9}\right)$ as high as 80 around at $5000 \mathrm{~K}$ were obtained by optimizing the BGYR wavelengths and their laser power densities.
\end{abstract}

\section{Introduction}

Laser lighting would offer significant advantages including high potential efficiencies at high current densities. Such white light sources are usually realized using phosphors excited by blue or violet LDs [1]. Therefore it is a common belief that white light produced by a set of lasers of different colors would not be of high enough quality for general illumination, in particular, practical values of color rendering index (CRI).

Meanwhile, the alternative high-luminance white light sources using only blue-green-red (BGR) three-color LDs are not suitable for lighting applications because of their very poor CRI. However, their very small diffusive illuminant area does not generate heat if the LDs are arranged apart. This feature is quite beneficial for realizing extremely light-weight, small and cold highluminance white light sources which have never been before.

Figure 1 shows a spectral plot and CRI data for a BGR ternary color system. The chromaticity coordinates are values of $x=0.3451, y=0.3516$. The peak wavelengths are $461 \mathrm{~nm}, 523 \mathrm{~nm}$, and $636 \mathrm{~nm}$. The $\mathrm{B} / \mathrm{G} / \mathrm{R}$ power distribution ratio is $0.42 / 0.71 / 1.0$.

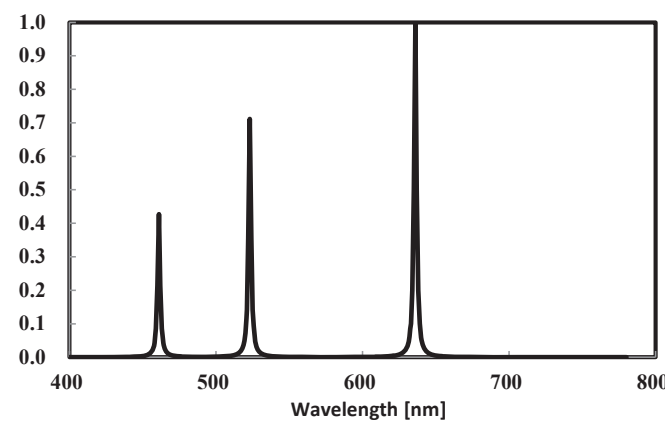

\begin{tabular}{|c|c|c|c|c|c|c|c|c|} 
R1 & R2 & R3 & R4 & R5 & R6 & R7 & R8 & Ra \\
\hline-33 & 59 & 50 & -44 & -13 & 38 & 11 & -124 & -7 \\
\hline R9 & R10 & R11 & R12 & R13 & R14 & R15 & & \\
\hline-364 & -6 & -80 & 49 & -11 & 64 & -48 & & \\
& & & & & & & &
\end{tabular}

Fig.1 BGR white light spectrum and its CRI values.
As in Fig.1, $R_{a}=-33$ is obtained for the BGR system. However, this value is too poor for general lighting applications. The value of $R_{9}$ is -364 and too bad.

To deploy this benefit to actual LD lighting applications, the BGR illuminants must evolve to the blue-greenyellow-red (BGYR) four-color system. Neumann et.al [2] and Kinoshita et.al [3] have reported the earlier distinguished research on the four-color LD illuminants. They demonstrated experimentally that CRI's values of a BGYR four-color LD illuminant were high $\left(R_{\mathrm{a}}=83\right.$ at CCT of $5665 \mathrm{~K}$ [2], $R_{\mathrm{a}}=90$ at $5500 \mathrm{~K}$ [3]). However, there are still many unclear points about the BGYR LD illuminants. For example, there are improvements in CRI values and the BGYR four-color LD wavelengths.

This paper describes the calculation results of finding improved CRI values of the BGYR four-color LD illuminants at the same CCT around $5000 \mathrm{~K}$, using the means to change the power ratio of their BGYR LDs and the chromaticity coordinates around $5000 \mathrm{~K}$.

\section{Simulation}

To obtain CRI values $\left(R_{1}-R_{15}\right)$, we use ColorCalculator (ver.7.77), provided by OSRAM Sylvania Inc. The average of $R_{1}-R_{8}$ is expressed as $R \mathrm{a}$ and The index of $\mathrm{R}_{9}$ means strong red. The line spectra of the LDs are assumed to be a Lorentzian shape with a FWHM of $2 \mathrm{~nm}$.

All chromaticities and illuminances were measured using a Konica Minolta CL-500A Spectrophotometer. Color rendering quality $\left(R_{a}, R_{9}\right)$ for the laser illuminants were determined by simulation.

For BGYR color system, the much higher CRI's values can be expected, because of increases of the degree freedom [3].

In the BGYR four-color system, there are so many calculation parameters, so the number of parameters was reduced by calculating the Yellow LD power and Blue LD power as the power ratio.

To find high CRI's values with the BGYR four-color LD illuminants at the same CCT around $5000 \mathrm{~K}$, The CRI values were calculated with the power ratio of yellow and blue LDs as parameters within the range of chromaticity coordinates of $5000 \mathrm{~K} \pm 400 \mathrm{~K}$. In the 
calculation within the chromaticity coordinate range, it was calculated every 0.001 chromaticity coordinates. CRI's values at all calculated chromaticity coordinates were obtained using ColorCalculaor.

The calculated chromaticity coordinate area is a range of rectangles diagonally $(\mathrm{x}, \mathrm{y})=(0.355,0.34)$ and $(0.365,0.37)$. The CRI values were calculated under the condition that the power ratio of the yellow and blue LDs was 1 . Figure 2 shows the simulation results of $R_{a}$ (a) and $R_{9}$ (b) of CRI. In Fig. 2 (a), the region with a high value of $R_{a}$ of 80 or more was uniformly wide. On the other hand, the region of 80 or more of $R_{9}$ had a narrow band shape. Because of the narrow band shape, it was found that the change increase and decrease of $\mathrm{R}_{9}$ was steep.
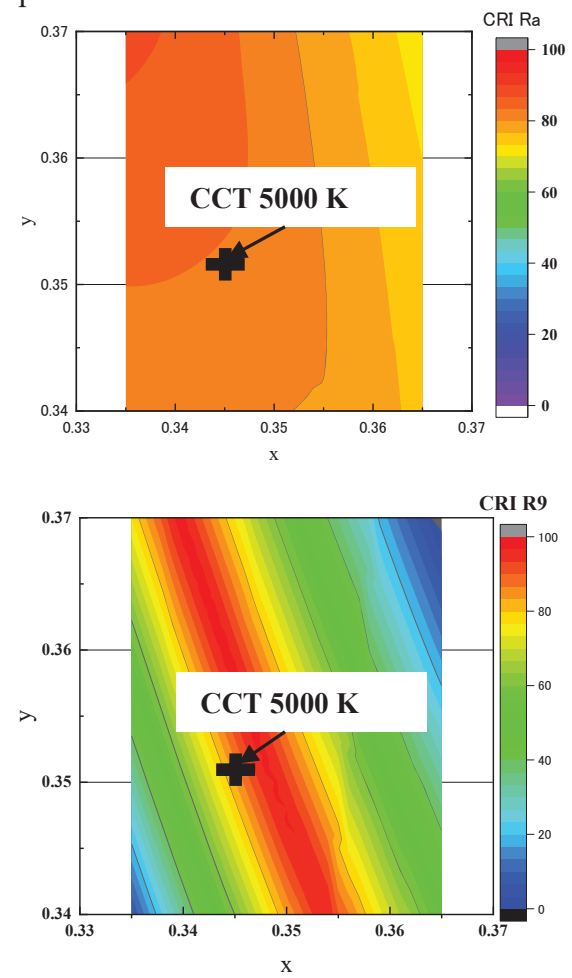

Fig. 2 Calculated $\mathrm{R}_{\mathrm{a}}(\mathrm{a})$ and $\mathrm{R}_{9}(\mathrm{~b})$ at $\mathrm{Y} / \mathrm{B}$ ratio $=1$.

Figure 3 shows the Yellow and Blue LDs power ratio dependence of the $R_{a}$ and $R_{9}$ values at the chromaticity coordinates of the cross mark CCT $5000 \mathrm{~K}(x=0.3451$,

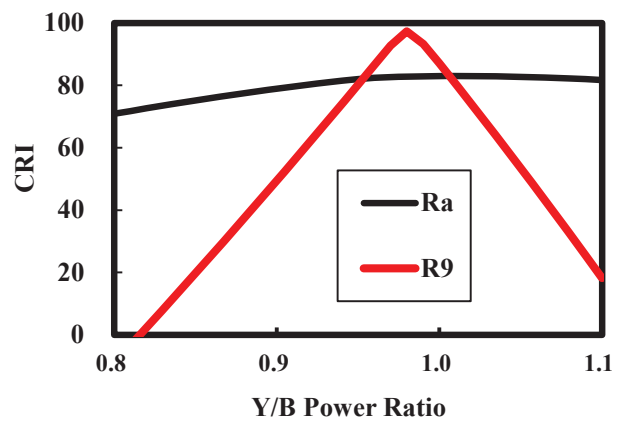

Fig. 3 Y/B power ratio dependence of $R_{a}$ and $R_{9}$ values. $y=0.3516$ ) shown in Figs. 2 (a) and 2 (b). In Fig. $3, \mathrm{R}_{\mathrm{a}}$ reached to the value of 80 near the $\mathrm{Y} / \mathrm{B}$ power ratio $=1$ and was saturated. $R_{9}$ 's value had a peak value of 98 at the $\mathrm{Y} / \mathrm{B}$ power ratio $=1$. From the results of the $\mathrm{Y} / \mathrm{B}$ power ratio dependencies of other CRIs, all CRI values were good when the $\mathrm{Y} / \mathrm{B}$ power ratio was 1.1. The CRI values were obtained by measuring under this condition.

\section{Results of CRI of a BGYR illuminant}

An example of the spectrum of the BGYR illuminant and the improved CRI values are shown in Fig. 4 for the peak wavelengths of $461 \mathrm{~nm}, 523 \mathrm{~nm}, 588 \mathrm{~nm}$ and 636 nm.

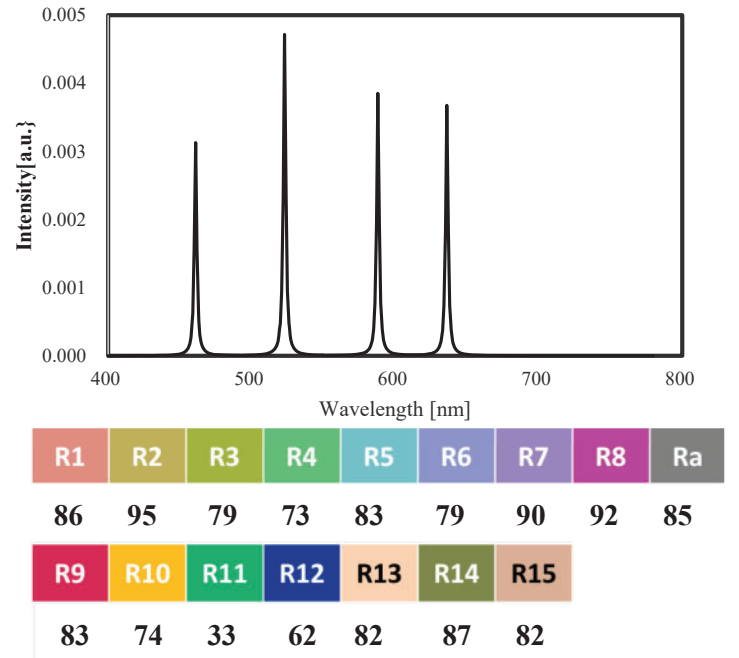

Fig.4 BGYR white light spectrum and its CRI values

The $\mathrm{B} / \mathrm{G} / \mathrm{Y} / \mathrm{R}$ power distribution ratio is 1.0/1.49/1.1/1.15. As in Fig.4, $R_{\mathrm{a}}=86$ and $R_{9},=83$ are obtained for the BGYR system. As a result, the BGYR LD illuminants are expected to use general lighting applications.

\section{Summary}

The CRI values of illuminants using BGYR four-color LDs could be improved around $5000 \mathrm{~K}$. As a result, both $R_{\mathrm{a}}$ and $\mathrm{R}_{9}$ with high values of $>80$ are obtained.

\section{References}

[1] K. Yagasaki et al., "Ce:YAG composite ceramic phosphors for laser lighting." The 9th Laser Display and Lighting Conference 2020, Yokohama, LDC 11-04.

[2]A. Neumann, J.J. Wierer, Jr., W. Davis, Y. Ohno, S.R. J. Brueck, and J. Y. Tsao, "Four-color laser white illuminant demonstrating high color-rendering quality", OPTICS EXPRESS, Vol.19, No.S4, A983- (2011)

[3]J. Kinoshita et al., "High Color Rendering Index using BGYR Four-Color Laser Illuminants" The 23th International Display Workshops in conjunction with Asia Display 2016, Fukuoka, Japan, PRJ1-4 (2016). 
LDC2021 | Oral Presentation

Imaging / Lighting

Wed. Apr 21, 2021 10:00 AM - 11:45 AM LDC (Online)

\section{[LDC-5-04] Color Shift Behavior at Image Pattern Edges of Raster-scan} RGB Mobile Laser Projectors

OJunichi Kinoshita ${ }^{1}$, Akira Takamori ${ }^{1}$, Kazuhisa Yamamoto ${ }^{1}$, Kazuo Kuroda ${ }^{2}$, Koji Suzuki ${ }^{3}$ (1.Osaka Universitry, 2.Utsunomiya University, 3.OXIDE Corporation)

Color shift behavior at image pattern edges of three rater-scan RGB mobile projectors is analyzed. The speckle noise effects are eliminated. Pure color shift is clearly picked up and analyzed. 


\title{
Color Shift Behavior at Image Pattern Edges of Raster-scan RGB Mobile Laser Projectors
}

\author{
Junichi Kinoshita ${ }^{1}$, Akira Takamori ${ }^{1)}$, Kazuhisa Yamamoto ${ }^{1)}$, \\ Kazuo Kuroda ${ }^{2}$, Koji Suzuki ${ }^{3)}$ \\ ${ }^{1)}$ Institute of Laser Engineering, Osaka University, 2-6 Yamada-oka, Suita, Osaka 565-0871, Japan, \\ phone number+81-6-6879-8958, fax number+81-6-6879-8958, and email jun1-istd@ile.osaka-u.ac.jp \\ ${ }^{2)}$ Center for Optical Research and Education, Utsunomiya University, ${ }^{3)}$ OXIDE Corporation
}

\begin{abstract}
Color shift behavior at image pattern edges of three rater-scan RGB mobile projectors is analyzed. The speckle noise effects are eliminated from the measured $2 D$ data by iris-opening of the speckle measurement equipment and averaging the residual speckle noise. Pure color shift is clearly picked up and analyzed.
\end{abstract}

\section{Introduction}

The image resolution under effects of speckle for rasterscan RGB mobile laser projectors has been measured and discussed by the authors $[1,2,3]$.

We found that the underlying dynamic color shift at the image pattern edges affects the resolution [1]. Examples of the edge color shift fully under effect of color speckle and that with minimized speckle effects are shown in the left and the right of Fig.1, respectively. The speckle effects are minimized by opening the iris of the speckle measurement equipment [4,5]. It is necessary for exactly analyzing the edge color shift behavior to pick up pure color shift data by eliminating the speckle noise that makes the color shift behavior unclear, as in Fig.1.

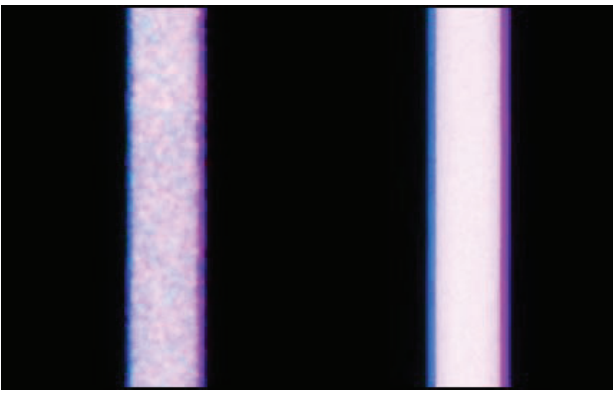

Fig. 1. Examples 2D data of edge color shift with color speckle effects (left) and that with minimized effects by opening the iris (right)

In this work, the pure color shift behavior at image pattern edges is analyzed in detail. The optical noise effects including speckle are eliminated by iris-opening of the speckle measurement equipment and also by averaging the residual speckle noise.

\section{Measurement preparation}

\subsection{Image patterns}

A vertical line pattern was used for analyzing the edge color shift. The pattern is a single line with a width of 18.85 pixels [1]. The pixel resolution of the three projectors under test shown later was $16: 9,1280 \times 720$ pixels (for input signal).

\subsection{Measurement setup}

Measurement setup is shown in Fig.2. The line pattern was projected on a standard diffusive screen, SRT-99120 (Labsphere) using three raster-scan RGB mobile laser projectors, $\# 1, \# 2$, and $\# 3$, one by one. The RGB wavelengths and the white chromaticity coordinates (CIE 1931) of each projector are summarized in Table 1.

The measured 2D data of the patterns were obtained by speckle measurement equipment, SM01VS11 (OXIDE Corporation) [4]. The actual dimensions were $D=710 \mathrm{~mm}, L=360 \mathrm{~mm}$, and $\theta=20.6^{\circ}$.

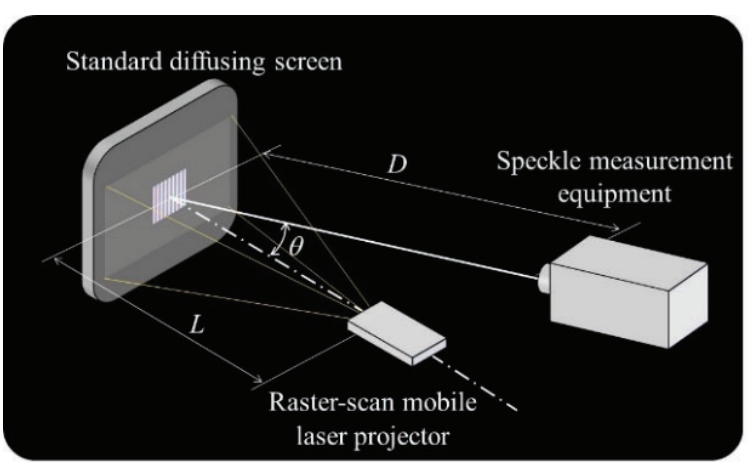

Fig. 2. Measurement setup.

Table 1. RGB wavelengths and white chromaticity coordinates (CIE 1931) of the projectors under test, \#1, 2, and 3.

\begin{tabular}{|c|c|c|c|c|c|}
\hline \multirow{2}{*}{ Projector\# } & \multicolumn{2}{|c|}{ Wavelength (nm) } & \multicolumn{2}{c|}{$\begin{array}{c}\text { White } \\
\text { chromaticity }\end{array}$} \\
\cline { 2 - 6 } & $\mathrm{R}$ & $\mathrm{G}$ & $\mathrm{B}$ & $x$ & $y$ \\
\hline$\# 1$ & 641 & 518 & 445 & 0.3027 & 0.2919 \\
\hline$\# 2$ & 644 & 518 & 445 & 0.3064 & 0.2920 \\
\hline$\# 3$ & 640 & 523 & 442 & 0.3016 & 0.3334 \\
\hline
\end{tabular}

\subsection{Elimination of speckle effects}

The speckle effects were minimized by fully opening the iris of the speckle measurement equipment [5]. The values of speckle contrast are suppressed to be around 0.02 . To eliminate the noise effects completely, all the 
measured data parallel to the line direction were averaged.

\section{Measurement results}

Horizontal plots of W, R, G, and B line outputs, and CIE 1931 chromaticity plots at left edge (L), center (C), and right edge (R) are shown in Figs.3, 4, and 5 for the projector $\# 1, \# 2$, and $\# 3$, respectively.

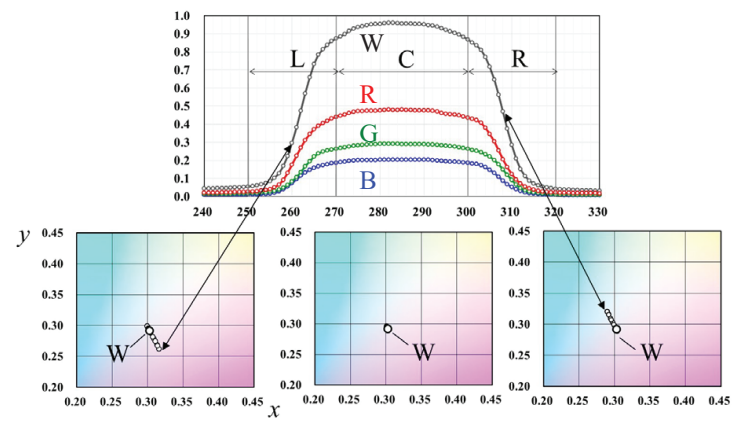

Fig. 3. Horizontal plots of W, R, G, and B line outputs, and CIE 1931 chromaticity plots at left edge, center, and right edge regions for the projector $\# 1$.

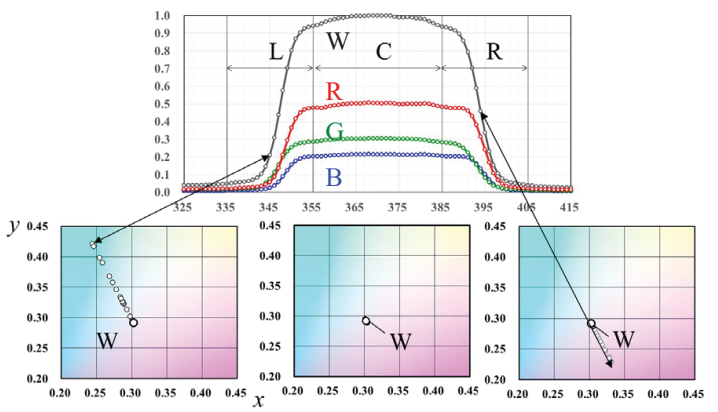

Fig. 4. Horizontal plots of W, R, G, and B line outputs, and CIE 1931 chromaticity plots at left edge, center, and right edge regions for the projector $\# 2$.

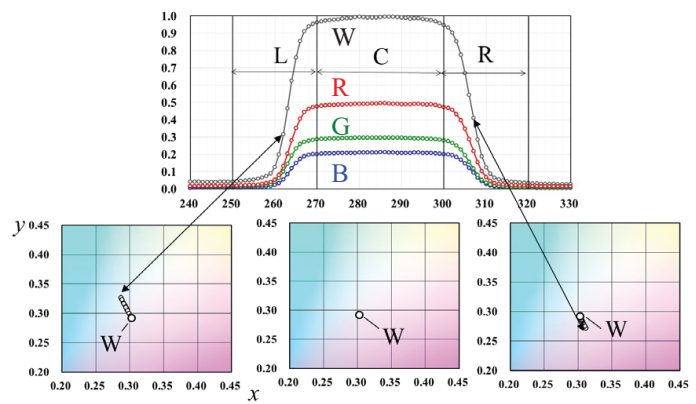

Fig.5. Horizontal plots of W, R, G, and B line outputs, and CIE 1931 chromaticity plots at left edge, center, and right edge regions for the projector \#3.

For the projector \#1, the position of the G output shifts rightward from the $\mathrm{R}, \mathrm{B}$ outputs. As a result, the left edge becomes purplish. The chromaticity of black and white regions is kept at the designed white chromaticity.
The purplish shift has its maximum in the point between the black and the white regions. At the right edge, the color shift becomes greenish.

The rise and fall at both edges are slowest for the projector \#1, which would make the resolution worse.

For the projector \#2, the position of the $G$ output shifts leftward from the R, B outputs. Therefore, the left edge becomes greenish and the right edge does purplish. It is in the reverse manner of the projector \#1. Furthermore, the color shift range is the largest among the three projectors under test.

For the projector \#3, the greenish shift at the left edge and the purplish shift at the right edge are in a similar manner to the projector \#2. However, the shift range is the smallest, showing the best performance of all.

\section{Discussion}

The color shift at the image pattern edges would be caused by the mismatch of the rise / fall timing among R, $\mathrm{G}, \mathrm{B}$ laser diodes.

\section{Conclusion}

Color shift behavior at image pattern edges of the three raterscan RGB mobile projectors is successfully analyzed. The speckle noise effects are eliminated from the measured 2D data by iris-opening of the speckle measurement equipment and averaging the residual speckle noise. The three projectors showed different behaviors depending on the degree of the mismatch of the rise / fall timing among R, G, B laser diodes.

\section{Acknowledgment}

The authors are grateful to Strategic International Standardization, Ministry of Economy, Trade and Industry, Japan.

\section{References}

[1] J. Kinoshita, K. Yamamoto, A. Takamori, K. Kuroda, K. Suzuki, "Visual resolution of raster-scan laser mobile projectors under effects of color speckle", Opt. Rev. 26(1), 187-200 (2019).

[2] J. Kinoshita, A. Takamori1, K. Yamamoto, K. Kuroda, K. Suzuki, K, Hieda, "Improved visual resolution measurement for laser displays based on eyediagram analysis of speckle noise",

[3] J. Kinoshita, A. Takamori1, K. Yamamoto, K. Kuroda, K. Suzuki, K, Hieda, "Speckled image resolution measured in nine regions on screen using raster-scan RGB laser mobile projector", The $27^{\text {th }}$ International Display Workshop. PRJ6-3 (2020)

[4] K. Suzuki, S. Kubota, "Understanding the exposuretime effect on speckle contrast measurements for laser displays", Optical Review 25 (1), 131-139 (2018).

[5] J. Kinoshita, K. Ochi, A. Takamori, K. Yamamoto, K. Kuroda, K. Suzuki, K. Hieda, "Color speckle measurement of white laser beam emitted from fiber output of RGB laser modules", Optical Review 26 (6), 720-728 (2019). 
LDC2021 | Oral Presentation

Imaging / Lighting

Wed. Apr 21, 2021 10:00 AM - 11:45 AM LDC (Online)

\section{[LDC-5-05] Ultra high resolution and VAC-free N3D technology and its applications}

OChin-Yung Hsieh ${ }^{1}$, Hao-Yu Liu' ${ }^{1}$, Ruey-Jer Weng ${ }^{1}$, Wei-Yi Lu ${ }^{1}$, Naoki Sumi ${ }^{2}$ (1.Innolux Corporation, 2.Innolux Japan K.K.)

In this paper, we introduce the Innolux N3D technology with ultra high resolution and its vergenceaccommodation conflict free feature. The N3D technology provide focus cues for nature viewing experience. Lot of 3D display applications can using this thehnology to improve its display quality. 


\title{
Ultra high resolution and VAC-free N3D technology and its applications
}

\author{
Chin-Yung Hsieh ${ }^{1}$, Hao-Yu Liu ${ }^{1 *}$, Ruey-Jer Weng ${ }^{1}$, Wei-Yi Lu ${ }^{1}$, Naoki Sumi ${ }^{2}$ \\ ${ }^{1}$ Technology Development Division, Innolux Corporation, No.3, Sec. 1, Huansi Rd., Tainan City, Southern Taiwan Science Park, \\ 74147, Taiwan (R.O.C.) Tel: +88665051888*E-mail: haoyu.liu@innolux.com \\ ${ }^{2}$ Advanced Technology, Innolux Japan K.K., 1-1-1, Ibukidai-higashi-machi, Nishi-ku,Kobe, Japan Tel: Tel: +81-78-991-5394
}

\begin{abstract}
In this paper, we introduce the Innolux N3D technology with ultra high resolution and its vergence-accommodation conflict free feature. The N3D technology provides focus cues for nature viewing experience. Lot of $3 D$ display applications can use this technology to improve its display quality.
\end{abstract}

\section{Introduction}

In recent years, numbers of Three-dimensional (3D) technology developed and some of them are already sold in store. It can simply seperate to two types, need wear glasses (stereoscope) or not (autostereoscope).

Virtual reality (VR) head mounted display (HMD) and color/polarizer/shutter glasses 3D display are the most common stereoscopic displays type, and there are a few products that using lenticular lens/barrier array type autostereoscopic display. But most of the commercial products only provide binocular images (LR images) for vergence.

The vergence and accommodation are the important mechanisms with human vision. Only providing vergence information will cause eye fatigue problem called vergence-accommodation conflict (VAC). One of the solution is light field type technology. The light field display, such as integral imaging, super multi-view (SMV)[2], holographic display, can control light direction in space, and with precalculated, it can produce light field like real objects.

Traditional SMV display consist of an flat display panel and additional lenticular lens/barrier array, Fig. 1 shows the schematic of SMV display, but satisfy super multi-view condition is more than two views points always pass through the pupil of each eye.

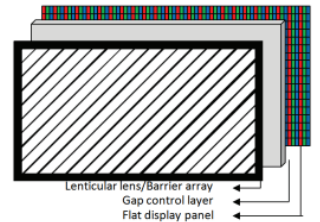

Fig. 1. Schematic of super multi-view display.

For so much view points, SMV display usually use lenticular lens/barrier array with slant angle technology to create several views. But it still cost lots of pixels on display for view creation. The simple solution for large view numbers creation is using the more high definition display with SMV technology.

In this paper, we introduce Innolux N3D display, it based on SMV technology, by using ultra high resolution and improvement algorithm with more efficient subpixels definition, so that can easily achieve VAC free condition for several applications.

\section{N3D and super multi-view technology.}

2.1 Vergence-accommodation conflict

In most stereoscopic display, lots of people will feedback eye fatigue or even dizzying. The main reason may be traced to the VAC effect.

In nature viewing, the vergence and accommodation distance are the same. But in most autostereoscopic displays, the vergence distance can be varied by stereo disparity, but eye still accommodate on the plane where display panel located.

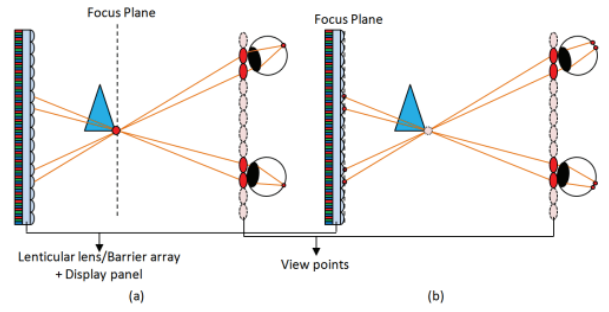

Fig. 2. Accommodation mechanism with super multi-view display: eyes focused at (a) 3D image, and (b) display screen.

\subsection{Focus cue production}

SMV display use the high density view points which the spacing between view points smaller than pupil size to create focus cues, Fig. 2. More than two view points receive by pupil, the image will need accommodation in distance different from original image plane. But due to the crosstalk of views, the pupil will receive wrong information, and it will cause image blur.

Innolux N3D display technology difine each ray direction of sub-pixels, by slant lenticular lens and ultra high resolution panel, each sub-pixel has its own ray direction, so we can create more than thousands direction in space, it can reconstruct 3D images with high accuracy and more details.

In SMV display, the 3D image based on the same points information is separated into several views, so the resolution drops. In $\mathrm{N} 3 \mathrm{D}$ display, the $3 \mathrm{D}$ image representation is based on localized region, and thousands of ray direction make left and right eyes see the part of images, with converge mechanism, the image will be merged in brain, Fig. 3(a), and still keep the focus cues for accommodation, Fig. 3(b), comparing to 
SMV display, N3D display can provide more 3D information with the same condition.

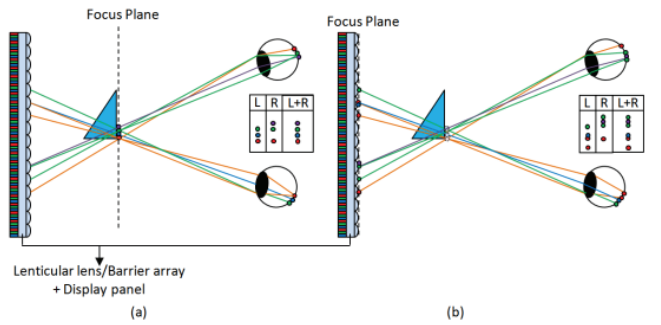

Fig. 3. Accommodation mechanism with N3D display: eyes focused at (a) 3D image, and (b) display screen.

2.2 Enhance resolution method with eye-tracking

In SMV display, when 3D image is displayed far from the display plane, the view to view crosstalk will let pupil received the other view points information which the pupil should not receive, with wrong image information, it will make image blur problem. The reduced view technology with eye tracking has been proposed for reducing blur and extend depth of field[3].

In N3D display, it has the similar technology with eye tracking. With eye position information, it will redefine the view direction with all views which views will be incoming eyes, although it is originally defined with other direction. So each eye will receive more effective views, the resolution will increase and the blur will be reduced.

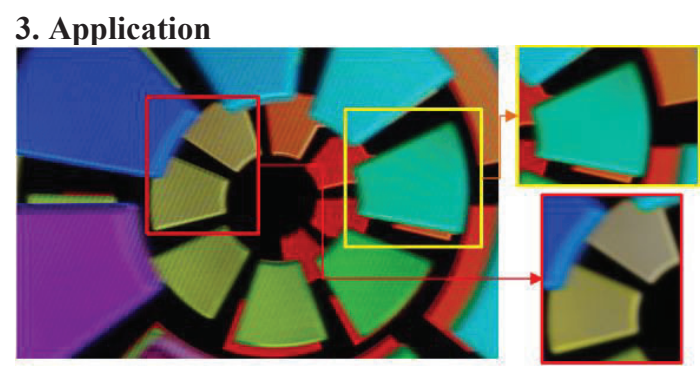

Fig. 4. N3D autostereoscopic display image which focus on display panel (Left), cyan region (right up), yellow region (right down) by camera with $4 \mathrm{~mm}$ aperture size.

\subsection{Autostereoscopic display}

With N3D technology, the autostereoscopic display has ultra high resolution and VAC free feature. Fig. 2 shows the N3D display based on 31.5 " 4k LCD panel, by using camera control the aperture to simulate image that eye received in different accommodation planes.

\subsection{Augmented reality (AR) head up display (HUD)}

In AR display, AR image and real objects needs to be shown at the same time. If the accommodation plane between AR images and real objects are different, it will cause uncomfortable feeling. With N3D technology, the accommodation plane of AR image can match to the real objects. Fig. 5 shows the N3D AR HUD.

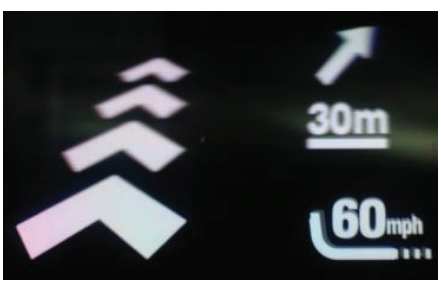

Fig. 5 N3D AR-HUD image which focus on "60mph".

\subsection{VR/AR HMD}

For HMD or stereoscopic display devices, VAC problem is well known issue. But, N3D technology can provide focus cues for each eyes. In VR device, with the optical design optimization and enhance algorithm, N3D VR can provide $0.3 \mathrm{~m} \sim$ infinity comfortable viewing depth range. Fig.6 shows the captured image from camera with N3D VR prototype. And this technology can be also extended to AR HMD device in future.

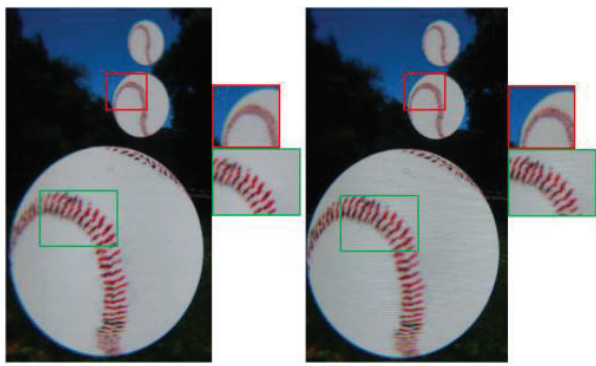

Fig. 6 N3D VR-HMD image in single eye view which focus on the lower ball (left), the middle ball (right).

\section{Conclusion}

In this paper, we introduce Innolux N3D technology with autostereoscopic display that can provide focus cues to solve VAC problem. And comparing to traditional SMV technology, it can provide more image information to human eyes. With eye tracking, it can provide more high resolution and reduce image blurring.

Moreover, N3D technology can be used in VR HMD/AR HUD for providing focus cues. In future work, we consider to apply $\mathrm{N} 3 \mathrm{D}$ technology to more applications.

\section{References}

[1] D. M. Hoffman, A. R. Girshick, K. Akeley, and M. S. Banks, "Vergence-accommodation conflicts hinder visual performance and cause visual fatigue," J. Vision Vol. 8(3):33, 1-30 (2008).

[2] Y. Takaki, "High-Density Directional Display for Generating Natural Three-Dimensional Images," Proc. IEEE Vol. 94(3), 654 - 663 (2006).

[3] J. Nakamura1, K. Tanaka, and Y. Takaki, "Increase in Depth of Field of Eyes Using Reduced-View Super MultiView Displays," Appl. Phys. Express Vol. 6(022501), 1-3 (2013). 
LDC2021 | Oral Presentation

\section{Light sources and components}

Wed. Apr 21, 2021 1:00 PM - 2:30 PM LDC (Online)

Click to the Online Room

[LDC-6-01 (Invited)] Nanowire LEDs with Ultrastable Emission Characteristics and Monolithically Integrated Multicolour Emission

OZetian $\mathrm{Mi}^{1}$, Xianhe Liu ${ }^{1}$, Yi Sun ${ }^{1}$, Yakshita Malhotra ${ }^{1}$, Yuanpeng $\mathrm{Wu}^{1}$

(1.University of Michigan)

1:00 PM - 1:30 PM

[LDC-6-02 (Invited)] Emission Characteristics of Random Lasers and Their Control

OTakashi Okamoto ${ }^{1}$ (1.Kyushu Institute of Technology)

1:30 PM - 2:00 PM

[LDC-6-03 (Invited)] Purcell-Effect-Enhanced Red Emission from Eu lons in GaN

Cavities

OYasufumi FUJIWARA ${ }^{1}$, Dolf TIMMERMAN ${ }^{1}$, Shuhei ICHIKAWA ${ }^{1}$, Jun

TATEBAYASHI $^{1}$ (1.Osaka University)

2:00 PM - 2:30 PM 
LDC2021 | Oral Presentation

Light sources and components

Wed. Apr 21, 2021 1:00 PM - 2:30 PM LDC (Online)

\section{[LDC-6-01 (Invited)] Nanowire LEDs with Ultrastable Emission} Characteristics and Monolithically Integrated Multicolour Emission

OZetian $\mathrm{Mi}^{1}$, Xianhe Liu ${ }^{1}$, Yi Sun ${ }^{1}$, Yakshita Malhotra ${ }^{1}$, Yuanpeng Wu ${ }^{1} \quad$ (1.University of Michigan)

We report on the design and demonstration of monolithically integrated InGaN/GaN micro-LEDs with multicolour emission using selective area molecular beam epitaxy, which exhibit unique properties including ultrastable operation, extremely narrow linewidth, and highly directional emission. 


\title{
Nanowire LEDs with Ultrastable Emission Characteristics and Monolithically Integrated Multicolour Emission
}

\author{
Zetian Mi, Xianhe Liu, Yi Sun, Yakshita Malhotra, and Yuanpeng Wu \\ Dept. of Electrical Engineering and Computer Science, University of Michigan, Ann Arbor, MI, USA. +1-(734)764-3963 \\ ztmi@umich.edu
}

\begin{abstract}
Selective area epitaxy is used to grow nanowires forming photonic crystal structures that exhibit unique properties including ultrastable operation, narrow linewidth, and directional emission. Monolithic integration of multicolour emission is also achieved in a single growth process from nanowires by controlling diameter-dependent In GaN composition in selective area epitaxy.
\end{abstract}

\section{Introduction}

High efficiency micro-scale light emitting diodes (LEDs) in the visible spectrum are the key for many applications including mobile displays, virtual/mixed/augmented reality, bio-photonics, and communications. To date, however, InGaN based micro-LEDs still have very low efficiency, particularly for green and red emission colours, which is mostly limited by high dislocation density, nonradiative surface recombination and poor current injection $[1,2]$. In this context, we have investigated the growth, electrical and optical properties of dislocation-free GaN/InGaN nanowires, and have demonstrated photonic crystal nanowire micro-LEDs with ultrastable operation and monolithically integrated multicolour emission.

\section{Theoretical design}

Nanowires are designed to be positioned in a triangular lattice with a lattice constant $a$ as shown in the inset of Fig. 1(a). The nanowires have identical diameter $d$. At $\Gamma$ point, the wavevector projected in any in-plane direction is zero, indicating wavevector pointing along the vertical direction and light being scattered in the vertical direction. This property is intriguing for enhancing the light extraction efficiency and vertical emission. Such structures are simulated to study how the wavelength of mode at $\Gamma$ point varies with the $\mathrm{d} / \mathrm{a}$ ratio. Here the lowest $\Gamma$ point with symmetric distribution is selected as the target [3]. The wavelength is calculated to vary from 544 $\mathrm{nm}$ to $595 \mathrm{~nm}$ as the $\mathrm{d} / \mathrm{a}$ ratio increases from 0.4 to 0.6 as shown in Fig. 1. By incorporating proper InGaN composition into nanowire arrays following this design, emission dominated by the $\Gamma$ point band edge mode is expected.

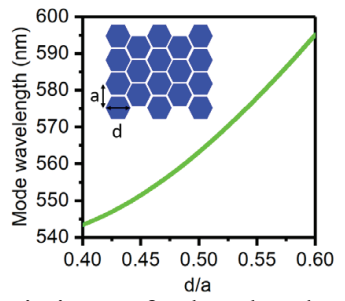

Fig. 1. Variation of the band edge mode wavelength at $\Gamma$ point with $\mathrm{d} / \mathrm{a}$ ratio. Inset: schematic of the nanowire photonic crystal with a triangular lattice.

\section{Experiment}

Our nanowire micro-LED structures are formed by selective area epitaxy on patterned substrates which have a thin $\mathrm{Ti}$ layer deposited and patterned on GaN-onsapphire templates. E-beam lithography and reactive ion etching technique are used to pattern the Ti layer. The nanowire micro-LEDs are fabricated with standard photolithography, dry etching, and contact metallization techniques. The nanowire arrays exhibit extremely high geometry uniformity and a pronounced and narrow resonant peak with a linewidth of $3.3 \mathrm{~nm}$ in the green spectrum as shown in Fig. 2(a). The device has excellent current-voltage characteristics, with a small turn-on voltage of $\sim 4 \mathrm{~V}$. Strong electroluminescence (EL) in the green spectrum is measured. The emission linewidth is only 3-3.7 $\mathrm{nm}$ and the peak position stays constant under widely varied injection current densities from $0.5 \mathrm{~A} / \mathrm{cm}^{2}$ to $210.8 \mathrm{~A} / \mathrm{cm}^{2}$ as shown in Fig. 2(b) [4]. This linewidth is significantly narrower than that of conventional planar quantum well LEDs. The quantum-confined Stark effect causing undesirable wavelength shift in conventional planar quantum well LEDs is also minimized as the wavelength is dominated by photonic crystal structure, which remains the same despite the variation of injection current.
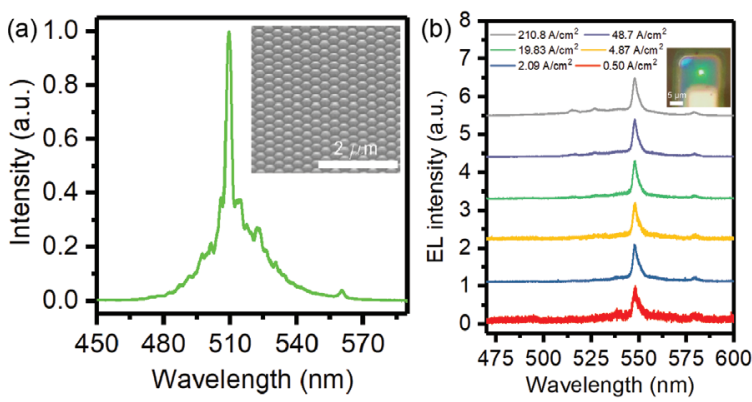

Fig. 2. (a) PL spectrum of the $\mathrm{GaN} / \mathrm{InGaN}$ nanowire photonic crystal structure. Inset: A scanning electron microscopy image of the $\mathrm{GaN} / \mathrm{InGaN}$ nanowires. (b) EL spectra of nanowire photonic crystal LED with stable emission spectra under various current densities. Inset: A photo of a micro LED under room light.

The relative external quantum efficiency (EQE) is computed by dividing the integrated EL intensity by the 
current. The EQE reaches maximum at $5 \mathrm{~A} / \mathrm{cm}^{2}$, which is similar to typical high efficiency blue LEDs. Only a moderate efficiency droop of $\sim 30 \%$ is observed at a high current density of more than $200 \mathrm{~A} / \mathrm{cm}^{2}$. Directional emission with a divergence angle of $\sim 10$ degrees is also realized as shown in Fig. 3(c) [4]. Such tailored emission properties and ultra-stable operation can greatly simplify the structure of optical systems and are ideally suited for near-eye micro-display applications.
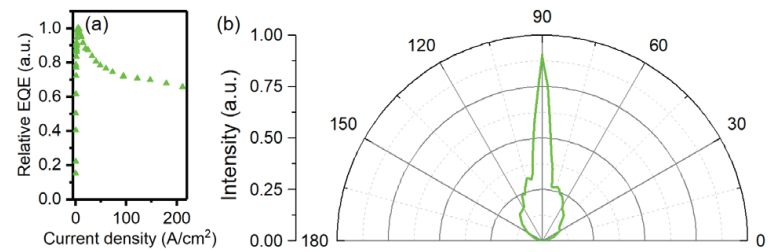

Fig. 3. (a) Variation of relative EQE with injection current density. (b) Angular distribution of far-field emission in a $10 \mathrm{~nm}$ spectral window around the peak wavelength.

As the InGaN composition depends on the nanowire diameter and spacing [5], multicolor emission can also be simultaneously achieved using a monolithic approach. Shown in Fig. 4(a) is a schematic of our devices. By defining different diameter and spacing for different arrays, the resultant $\mathrm{InGaN}$ compositions are different because the incorporation of In heavily depends on the geometry parameters of the nanowire array. Larger diameter tends to incorporate more In, thus leading to emission of longer wavelength. Shown in Fig. 4(b) are the EL spectra from different devices achieved in a single growth process. The color ranges from green to orange. One of the spectra measured from a $3 \mu \mathrm{m} \times 3$ $\mu \mathrm{m}$ micro LED exhibits a narrow emission peak at 561 $\mathrm{nm}$ due to photonic crystal structure. The linewidth is as narrow as $10 \mathrm{~nm}$. All the measured peak positions are compared with the simulation result in Fig. 5. The $3 \mu \mathrm{m}$ $\times 3 \mu \mathrm{m}$ micro LED is indicated by the arrow. This data point is the closest to the simulation result. Other devices deviate from the theoretical prediction and thus do not exhibit narrow emission peak from photonic crystal structure. It is expected that such unique emission spectrum can also be simultaneously achieved at other wavelengths upon optimization of the diameter and the spacing for the respective $\mathrm{InGaN}$ composition.

\section{Conclusions}

In conclusion, we have demonstrated micro LEDs with unique emission properties enabled by nanowire photonic crystal structure and integrated such devices with multicolor emission in a single growth process. The intriguing features exhibited by our devices, including extraordinary stable emission peak wavelength, narrow spectral linewidth, emission directionality, moderate efficiency droop, and multicolor emission, are essential for next generation ultrahigh resolution displays and near-eye display applications. Given the stable operation determined by photonic crystal and scalable growth and fabrication process, further enhancement of EQE would enable for high power operation. The nanowire photonic crystal structure proves to be a viable route for advancing display technology.
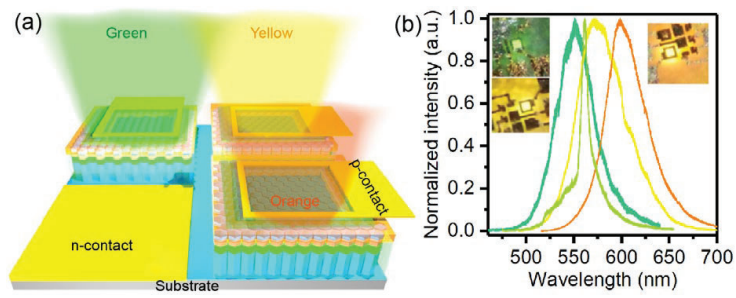

Fig. 4. (a) Schematic of the monolithically integrated $\mathrm{GaN} / \mathrm{InGaN}$ nanowire LEDs. (b) EL spectra of the monolithically integrated multicolor nanowire LEDs simultaneously achieved with photonic crystal effect. Inset: Photos of three green, yellow, and orange LEDs under room light

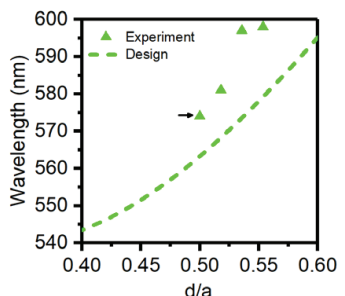

Fig. 5 Comparison between wavelength obtained from simulation and peak position measured from experiment. The data point indicated by the arrow corresponds to the device with narrow pronounced peak from photonic crystal structure.

\section{Acknowledgment}

The work was supported by NS Nanotech, Inc.

\section{References}

[1] F. Olivier, S. Tirano, L. Dupré, B. Aventurier, C. Largeron, and F. Templier, "Influence of size-reduction on the performances of GaN-based micro-LEDs for display application," J. Lumin. 191, 112-116 (2017).

[2] M. Minami, S. Tomiya, K. Ishikawa, R. Matsumoto, S. Chen, M. Fukasawa, F. Uesawa, M. Sekine, M. Hori, and T. Tatsumi, "Analysis of GaN Damage Induced by Cl2/SiCl4/Ar Plasma," Jpn. J. Appl. Phys. 50, 08 JE03 (2011).

[3] A. Yanagihara, S. Ishizawa, and K. Kishino, "Directional radiation beam from yellow-emitting InGaN-based nanocolumn LEDs with ordered bottom-up nanocolumn array," Appl. Phys. Express 7, 112102 (2014).

[4] X. Liu, Y. Wu, Y. Malhotra, Y. Sun, and Z. Mi, "Micrometer scale InGaN green light emitting diodes with ultra-stable operation," Appl. Phys. Lett. 117, 011104 (2020).

[5] H. Sekiguchi, K. Kishino, and A. Kikuchi, "Emission color control from blue to red with nanocolumn diameter of InGaN/GaN nanocolumn arrays grown on same substrate," Appl. Phys. Lett. 96, 231104 (2010). 
LDC2021 | Oral Presentation

Light sources and components

Wed. Apr 21, 2021 1:00 PM - 2:30 PM LDC (Online)

\section{[LDC-6-02 (Invited)] Emission Characteristics of Random Lasers and Their Control}

OTakashi Okamoto ${ }^{1}$ (1.Kyushu Institute of Technology)

Random lasers are mirrorless lasers comprising scatterers and an active medium. Multiple scattering of light provides the feedback mechanism for lasing. In this talk, I will present the mechanisms and properties of a random laser and emission control methods. 


\title{
Emission Characteristics of Random Lasers and Their Control
}

\author{
Takashi Okamoto \\ Faculty of Computer Science and Systems Engineering, Kyushu Institute of Technology, 680-4 Kawazu, Iizuka, \\ Fukuoka 820-8502, Japan, 0948-29-7725, okamoto@ces.kyutech.ac.jp
}

\begin{abstract}
Random lasers are mirrorless lasers comprising scatterers and an active medium. Multiple scattering of light provides the feedback mechanism necessary for lasing. In this talk, I will present the fundamental mechanisms and properties of a random laser and emission control methods.
\end{abstract}

\section{Introduction}

Light scattering is one of the most typical phenomena in daily life. However, it is often detrimental to various optical devices, particularly lasers. Random lasers are unique in that scattering is crucial in producing stimulated emission [1-4]. In this talk, I will provide an overview of the physics of random lasers and the characteristics of their light emission. Furthermore, I will present several methods for controlling the properties of random lasing.

\section{Mechanism of random laser}

A conventional laser comprises a gain material that amplifies light as well as a cavity that partially traps light and selects resonant wavelengths. In a random laser, a regular cavity is replaced by a disordered medium in which light is multiply scattered. Multiple scattering offers a long light path, resulting in stimulated emission. The following criteria for a random laser are proposed [1]: (1) light is multiply scattered owing to randomness and amplified by stimulated emission, and (2) a threshold exists because of multiple scattering above which the total gain is larger than the total loss. Figure 1 shows a schematic diagram of random lasing, where light is amplified through multiple scattering.

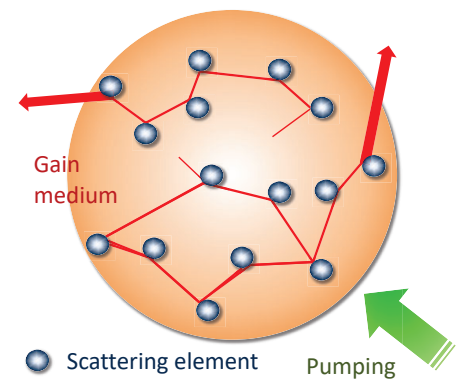

Fig. 1. Schematic diagram of random lasing.

Two classes of random lasing exist, and they are distinguished by whether resonance is involved in light emission.

2.1 Incoherent random lasing

As shown in Fig. 1, spontaneously emitted light is amplified by stimulated emission as it is multiply scattered in an active medium. A random gain medium is typically an open system, and the light escapes easily from the medium. However, when the gain exceeds a certain loss value, the lasing threshold is reached, and the linewidth of the emission spectrum narrows significantly. This type of lasing is known as incoherent random lasing. As shown in Fig. 2(a), the spectrum has a single peak, and the peak wavelength coincides with that at the maximum of the gain spectrum.

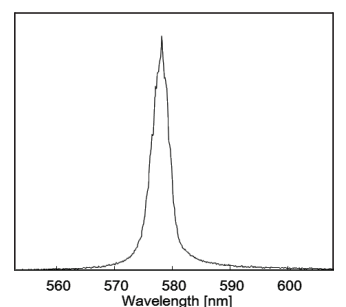

(a)

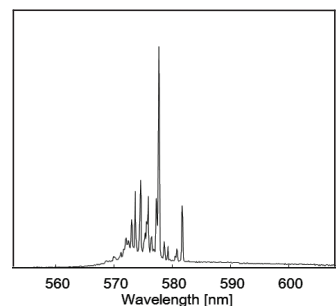

(b)
Fig. 2. Typical emission spectra of (a) incoherent random lasing and (b) coherent random lasing.

\subsection{Coherent random lasing}

Figure 2(b) shows the emission spectrum of coherent random lasing, in which several peaks with a subnanometer linewidth exist. The diagram in Fig. 1 suggests that ring cavities are formed in the scattering medium. However, this simple illustration can only be applied to strongly scattering media. Furthermore, narrow spectral peaks are observed in weakly scattering systems in which no localized mode exists. The modes of coherent random lasing can be extended over the entire medium; they are leaky and their Q (quality) factors are low [5]. This is in contrast to conventional lasers, which have definite cavities and localized modes.

\section{Characteristics of light emission}

Conventional lasers typically exhibit high temporal and spatial coherence. The coherence of random lasers is generally lower than that of conventional ones but can be adjusted by changing various physical parameters, such as the size, density, and refractive index of scatterers, the size of the random medium, the gain length of the active medium, and the area and shape of the optical pump.

Temporal coherence is directly related to the emission spectrum. Coherent and incoherent laser emissions can be selected by adjusting the values of the parameters mentioned above. Typically, the temporal coherence of 
random lasers is shorter than that of LEDs but longer than that of single-mode lasers. A random laser with a diameter of a few micrometers exhibits a narrow single-peak spectrum with a linewidth of $0.2 \mathrm{~nm}$ [6].

One of the most interesting characteristics of random lasers is that they exhibit low spatial coherence while maintaining relatively high temporal coherence. This property is advantageous for imaging applications because typical lasers with high spatial coherence induce speckle patterns, which degrade the image. A random laser comprises transverse modes that are independent of one another. Images without speckles have been obtained experimentally using a random laser with colloidal solutions of polystyrene spheres and laser dye [7].

\section{Control of random lasing}

A random laser is a disordered system intrinsically; hence, new approaches are necessitated to control laser emission. Next, I present some methods that have been proposed to provide the desired emission characteristics.

\subsection{Design of random structure}

The size, shape, refractive index, and spatial distribution of scatterers in gain media strongly affects random laser action because the optical feedback is provided by multiple scattering of light. Mie resonances of randomly assembled monodisperse spheres allow us to control the emission spectrum by changing the diameter and refractive index of the spheres [8]. The efficiency of random lasing can be improved by introducing inhomogeneity in the distribution of scatterers. In the random gain medium of monodisperse particles, a defect focuses and enhances the light in the region around it [9]. Furthermore, the clustering of particles affects the efficiency of random lasing; the lasing threshold decreases as the degree of particle aggregation is increased [10]. Spacer particles (non-scattering regions) dispersed in random gain media increase the spectral peak intensity for both coherent and incoherent random lasing [11]. This peak enhancement occurs even when the spacer particles have no gain [12].

The design of the structure of a random laser medium has been investigated [13]. To simplify the complicated random structures of conventional random lasers, a twodimensional random medium with a checkerboard pattern structure was employed. The simulation results show that the emitted light can be concentrated within a certain wavelength range using the direct binary search algorithm.

\subsection{Optimization of pump light distribution}

The spatial distribution of the pump light changes the spectrum and direction of random laser emission. The tuning of the emission spectrum $[14,15]$ and direction [16] is enabled by the design of a pattern for pump intensity distributions. A single sharp spectral peak or directional control of laser emission can be realized using this method, although the pump pattern should be optimized for each random laser medium. Furthermore, different lasing modes can be invoked by changing the shape of the pump [17].

\section{Conclusion}

Since Lawandy's pioneering work [4], random lasers have been investigated as not only as a new light source, but also for various purposes, such as security, environmental sensing, and biomedical sensing and diagnostics. This indicates that random lasers exhibit significant potential in extending the field of laser applications. Most studies are still in the research stage, and it is anticipated that they will be put to practical use in various fields in the near future.

\section{References}

[1] D. S. Wiersma, "The physics and applications of random lasers," Nat. Phys. 4, 359-367 (2008).

[2] F. Luan, B. Gu, A. S. L. Gomes, K.-T. Yong, S. Wen and P. N. Prasad, "Lasing in nanocomposite random media," Nano Today 10, 168-192 (2015).

[3] M. A. Noginov, Solid-State Random Lasers (Springer, New York, 2005).

[4] N. M. Lawandy, R. M. Balachandran, A. S. L. Gomes and E. Sauvain, "Laser action in strongly scattering media," Nature 368, 436-438 (1994).

[5] J. Andreasen, A. A. Asatryan, L. C. Botten, M. A. Byrne, H. Cao, L. Ge, L. Labonté, P. Sebbah, A. D. Stone, H. E. Türeci and C. Vanneste, "Modes of random lasers," Adv. Opt. Photon. 3, 88-127 (2011).

[6] H. Cao, J. Y. Xu, E. W. Seelig and R. P. H. Chang, "Microlaser made of disordered media," Appl. Phys. Lett. 76, 2997-2999 (2000)

[7] B. Redding, M. A. Choma and H. Cao, "Speckle-free laser imaging using random laser illumination," Nat. Photon. 6, $355-359$ (2012).

[8] S. Gottardo, R. Sapienza, P. D. García, A. Blanco, D. S. Wiersma and C. López, "Resonance-driven random lasing," Nat. Photon. 2, 429-432 (2008).

[9] H. Fujiwara, R. Niyuki, Y. Ishikawa, N. Koshizaki, T. Tsuji and K. Sasaki, "Low-threshold and quasi-single-mode random laser within a submicrometer-sized $\mathrm{ZnO}$ spherical particle film," Appl. Phys. Lett. 102, 061110 (2013).

[10] M. Leonetti and C. Lopez, "Random lasing in structures with multi-scale transport properties," Appl. Phys. Lett. 101, 251120 (2012)

[11] T. Okamoto and R. Yoshitome, "Random lasing in dyedoped polymer random media with a bubble structure," J. Opt. Soc. Am. B 34, 1497-1502 (2017).

[12] T. Okamoto and M. Mori, "Random laser action in dyedoped polymer media with inhomogeneously distributed particles and gain," Appl. Sci. 9, 3499 (2019).

[13] T. Okamoto and K. Imamura, "Designing random structures for random laser media," Tech. Digest. OIE '19, 87-88 (2019).

[14] M. Leonetti and C. López, "Active subnanometer spectral control of a random laser," Appl. Phys. Lett. 102, 071105 (2013).

[15] N. Bachelard, S. Gigan, X. Noblin and P. Sebbah, "Adaptive pumping for spectral control of random lasers," Nat. Phys. 10, 426-431 (2014).

[16] T. Hisch, M. Liertzer, D. Pogany, F. Mintert and S. Rotter, "Pump-controlled directional light emission from random lasers," Phys. Rev. Lett. 111, 023902 (2013).

[17] M. Leonetti, C. Conti and C. Lopez, "The mode-locking transition of random lasers," Nat. Photon. 5, 615-617 (2011). 
LDC2021 | Oral Presentation

Light sources and components

Wed. Apr 21, 2021 1:00 PM - 2:30 PM LDC (Online)

\section{[LDC-6-03 (Invited)] Purcell-Effect-Enhanced Red Emission from Eu Ions in GaN Cavities}

OYasufumi FUJIWARA ${ }^{1}$, Dolf TIMMERMAN ${ }^{1}$, Shuhei ICHIKAWA ${ }^{1}$, Jun TATEBAYASHI ${ }^{1}$ (1.Osaka University)

Luminescence properties of a Eu-doped GaN microdisk cavity and a two-dimensional photonic crystal

nanocavity are demonstrated. The resonant modes are coupled with indirectly excited Eu ${ }^{3+}$ ions and enhance drastically Eu emission, which is due to the Purcell effect. 


\title{
Purcell-Effect-Enhanced Red Emission from Eu Ions in GaN Cavities
}

\author{
Yasufumi FUJIWARA, Dolf TIMMERMAN, Shuhei ICHIKAWA, and Jun TATEBAYASHI \\ Graduate School of Engineering, Osaka University, 2-1 Yamadaoka, Suita, Osaka 565-0871, Japan
}

\begin{abstract}
Luminescence properties of a Eu-doped GaN microdisk cavity and a two-dimensional photonic crystal nanocavity are demonstrated. The resonant modes are coupled with indirectly excited Eu ${ }^{3+}$ ions and enhance drastically Eu emission, which is due to the Purcell effect. This observation gives us a clue for further development of a red light-emitting diode using Eu-doped GaN.
\end{abstract}

\section{Introduction}

There has been a strong demand to develop red lightemitting diodes (LEDs) using nitride semiconductors for monolithic integration of the three primary colors (RGB), resulting in the realization of a nitride-based display with ultrasmall-size, full-color, and high-resolution. We have worked on the development of semiconductors intracenter photonics. This novel photonics uses the intra- $4 f$ shell transitions of rare-earth ions doped in semiconductors. In 2009, we invented a narrow-band red LED using Eu-doped GaN (GaN:Eu) [1]. A main emission line with a half width of less than $1 \mathrm{~nm}$ is observed at $621 \mathrm{~nm}$, which can be assigned to the ${ }^{5} D_{0}-$ ${ }^{7} F_{2}$ transition of $\mathrm{Eu}^{3+}$ ions. The wavelength is extremely stable against the ambient temperature. Due to optimization of the device processing, the output power of the LED has been increasing steadily to $1 \mathrm{~mW}$ [2]. High external quantum efficiency over $9 \%$ is also obtained at low current injection, which is very attractive to next-generation micro-LED displays.

In general, the output power of the red LED is limited by two factors; energy transfer to Eu ions from the $\mathrm{GaN}$ host, and radiative recombination probability in $\mathrm{Eu}$ ions. The energy transfer efficiency has been found to depend strongly on defect environments around Eu ions [3], which can be artificially influenced by impurity codoping [4]. A relatively long radiative lifetimes of the $\mathrm{Eu}$ emission in $\mathrm{GaN}: \mathrm{Eu}(\sim 240 \mu \mathrm{s})$ is another limiting factor. According to the Fermi's golden rule, modifying the spontaneous emission rate of $\mathrm{Eu}$ ions can be achieved by increasing the photonic density of states at the frequency of spontaneous emission. We have boosted the output power by actively manipulating radiative recombination probability of the Eu emission, which can be achieved through control of their photon fields in micro- and nano-cavities. In this talk, drastic enhancement of Eu emission due to the Purcell effect is demonstrated in a microdisk (MD) cavity and a twodimensional photonic crystal (2D-PhC) nanocavity using $\mathrm{GaN}: \mathrm{Eu}$.

\section{Microdisk cavity}

Figure 1 shows PL spectra taken for three different MDs fabricated with a design diameter of $1.57 \mu \mathrm{m}$ [5]. Due to small variations resulting from the fabrication process it can be observed that they have different resonant modes. We designate the three MDs as off-resonance (MD0) and on-resonance (MD1 and MD2). The inset shows the ratio of on- and off-resonance structures for MD1 and MD2, which both exhibit two peaks spaced $4.5 \mathrm{~nm}$ apart.

Time-resolved photoluminescence was measured by collection of light in the plane of the microdisk. For the on-resonance MDs, the intensity was determined around the peak of the resonance, while for the off-resonance $\mathrm{MD}$, it was taken around the peak of the emission spectrum. The inset of Fig. 2 shows all three transients. The off-resonance MD shows single exponential behavior with a decay time of $239.8 \mu \mathrm{s}$, which is

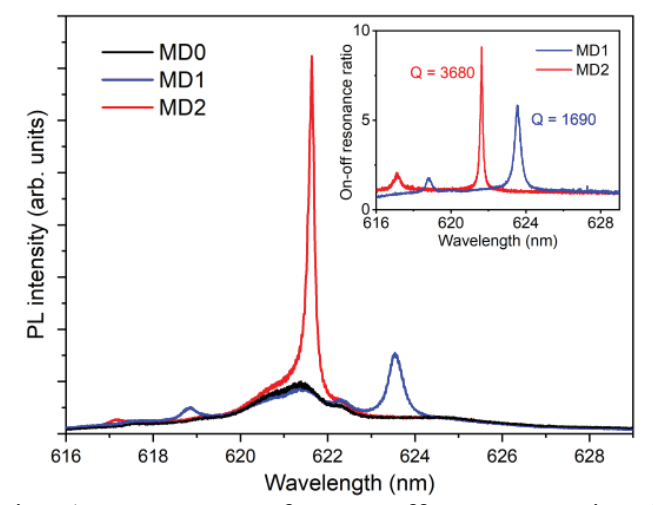

Fig. 1. PL spectra for an off-resonant microdisk (MD0) and two resonant microdisks (MD1 and MD2). Inset: Ratio of spectra of the on- and offresonance microdisks.

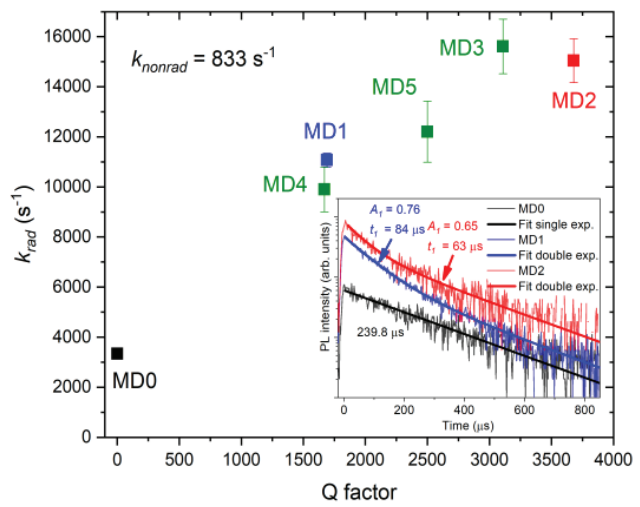

Fig. 2. Radiative rate determined from the fast component of the PL transients as a function of $Q$ factor for 6 MDs. Inset: PL decay transients and fits for the MDs. 
identical to that of an unpatterned layer. The emission from the on-resonance MDs could be well-fitted with a bi-exponential decay function, where the time constant of the slower component was fixed to $239.8 \mu \mathrm{s}$ representing the emission from $\mathrm{Eu}^{3+}$ ions that is not coupled to the cavity resonance. We find that for MD1 and MD2 about $70 \%$ of the emission has a faster decay time.

The PL lifetime at RT is approximately $80 \%$ of that at cryogenic temperatures $(\sim 300 \mu \mathrm{s})$. It means that the non-radiative lifetime at RT is $1.2 \mathrm{~ms}$. This value was used to determine the radiative lifetime of the $\mathrm{Eu}^{3+}$ ions coupled to the MD resonance from the fast component in the PL lifetime. The radiative rate $k_{\text {rad }}=1 / \tau_{\text {rad }}$ for these structures and three other similarly sized MDs are shown in Fig. 2 as a function of the cavity $Q$ factor. A clear trend can be observed, which is expected for the Purcell effect enhancing the radiative rate of $\mathrm{Eu}^{3+}$ ions.

\section{Two-dimensional photonic crystal nanocavity}

A L7- type 2D-PhC nanocavity with a triangular lattice pattern of hexagonal air holes was fabricated. Figure 3(a) shows the PL spectra of the nanocavities with radius-toperiod ratio $(r / a)$ of 0.27 and 0.32 [6]. The black dotted curve is a 2.1-times-enlarged PL spectrum of the nonpatterned GaN:Eu area. For L7 nanocavities, two obvious resonant peaks, corresponding to the fundamental (M1) and second modes (M2), were observed. Energy separation between M1 and M2 modes $(\sim 15 \mathrm{meV})$ indicates that the respective cavity modes are basically coupled to different Eu centers. Moreover, the resonant peaks moved to shorter wavelengths as $r / a$ increased.

The PL intensity ratio was obtained from the nanocavity with $r / a$ of 0.32 . There is a nearly constant value of $\sim 2.2$, and the maximum 16-fold enhancement was achieved by coupling with the M1 mode at a wavelength of $623.62 \mathrm{~nm}$. At the same time, a 4.1-fold enhanced emission at $617.60 \mathrm{~nm}$ coupled with the M2 mode was also observed. The $Q$-factors of M1 and M2 modes were experimentally estimated by the relationship of $Q=\lambda / \Delta \lambda$ to be 5400 and 1800 , respectively. The $Q-$ factor of 5400 is comparable to those of state-of-the-art nitride-based 2D-PhC nanocavities with embedded active layers in the blue/ultraviolet region.

\section{Conclusions}

The red LED using GaN:Eu has been steadily growing up. We demonstrated the 10.9-fold enhanced Eu emission from a LED with a vertical cavity [7]. However, the enhancement was due to improved light extraction efficiency because the $Q$-factor was not so high ( 80$)$. In the MD with the higher Q-factor, the emission intensity was much enhanced by increased radiative recombination probability due to the Purcell effect. Similar enhanced behavior was also observed in the 2D$\mathrm{PhC}$ nanocavity. These results indicates that precise control of photon fields is important for further development of our red LEDs.

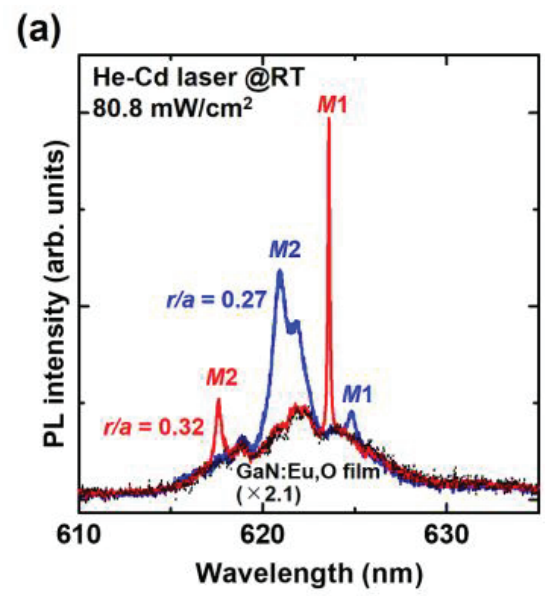

(b)

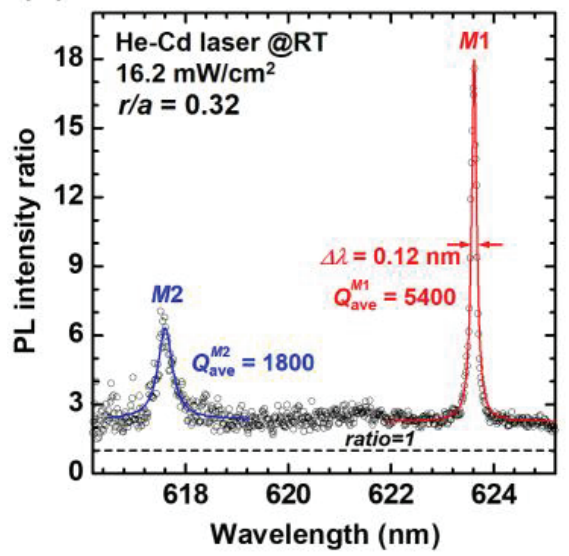

Fig. 3. (a)PL spectra of L7-type 2D-PhC nanocavities with $r / a$ of 0.27 and 0.32 . Black dotted curve is a 2.1-times-enlarged PL spectrum of the non-patterned $\mathrm{GaN}: \mathrm{Eu}$ area. (b)PL intensity ratio obtained from an L7 nanocavity with $r / a$ of 0.32 .

\section{Acknowledgment}

This work was partially supported by JSPS KAKENHI Grant Nos.18H05212.

\section{References}

[1] A. Nishikawa, T. Kawasaki, N. Furukawa, Y. Terai, and Y. Fujiwara, Applied Physics Express 2, 071004 (2009).

[2] B. Mitchell, V. Dierolf, T. Gregorkiewicz, and Y. Fujiwara, Journal of Applied Physics 123, 160901 (2018).

[3] R. Wakamatsu, D.-g. Lee, A. Koizumi, V. Dierolf, and Y. Fujiwara, Journal of Applied Physics 114, 043501 (2013).

[4] D. Lee, A. Nishikawa, Y. Terai, and Y. Fujiwara, Appl. Phys. Lett. 100, 171904 (2012).

[5] D. Timmerman, Y. Matsude, Y. Sasaki, S. Ichikawa, J. Tatebayashi, and Y. Fujiwara, Phys. Rev. Applied 14, 064059 (2020).

[6] S. Ichikawa, Y. Sasaki, T. Iwaya, M. Murakami, M. Ashida, D. Timmerman, J. Tatebayashi, and Y. Fujiwara, submitted to Phys. Rev. Appl.

[7] T. Inaba, J. Tatebayashi, K. Shiomi, D. Timmerman, S. Ichikawa, and Y. Fujiwara, ACS Appl. Electron. Mater. 2, $732(2020)$ 
LDC2021 | Oral Presentation

\section{Laser technology for automotive applications 1}

Wed. Apr 21, 2021 2:45 PM - 3:45 PM LDC (Online)

Click to the Online Room

[LDC-7-01 (Invited)] Laser Crystal Phosphor Automobile Headlight Integrated with Beam Control and LiDAR

OKenneth Li ${ }^{1}$, Y. P. Chang ${ }^{2}$ (1.Optonomous Technologies Inc., 2.Taiwan Color Optics, Inc.)

[LDC-7-02]

2:45 PM - 3:15 PM

[LDC-7-03]

Improvement of Color LiDAR with RGB visible single-mode laser diodes

OMasato Ishino ${ }^{1}$, Tomoyuki Ohashi ${ }^{1}$, Hiroshi Fuji ${ }^{1}$, Kana Fujioka ${ }^{1}$, Kazuhisa

Yamamoto $^{1}$ (1.Osaka University)

3:15 PM - 3:30 PM

Thermal property of AIN-Ce:YAG composite ceramics phosphor for laser lighting

OTakuya Sawada ${ }^{1}$, Hiroshi Huji ${ }^{1}$, Kenta Yagasaki ${ }^{1}$, Hisashi Minemoto ${ }^{1}$, Yukio Manabe ${ }^{1}$, Kana Fujioka ${ }^{1}$, Kazuhisa Yamamoto ${ }^{1}$ (1.Institute of Laser Engineering, Osaka University)

3:30 PM - 3:45 PM 
LDC2021 | Oral Presentation

Laser technology for automotive applications 1

Wed. Apr 21, 2021 2:45 PM - 3:45 PM LDC (Online)

\section{[LDC-7-01 (Invited)] Laser Crystal Phosphor Automobile Headlight Integrated with Beam Control and LiDAR}

OKenneth $\mathrm{Li}^{1}$, Y. P. Chang ${ }^{2}$ (1.Optonomous Technologies Inc., 2.Taiwan Color Optics, Inc.)

This invited paper presents our current research in the subject of integrated Beam Control Headlight and LiDAR, which includes a DMD pixel-based system and a polygon scanning mirror-based system. Single crystal phosphor is used providing high efficiency and reliability to the system. The goal is to provide highly reliable, compact, and low-cost systems to be introduced into the mass market for autonomous vehicles. 


\title{
Invited Paper
}

\section{Laser Crystal Phosphor Automobile Headlight Integrated with Beam Control and LiDAR}

\author{
Kenneth $\mathrm{Li}^{1)}$, Yung Peng Chang ${ }^{2)}$ \\ 1) Optonomous Technologies Inc., 30330 Rainbow View Ct., Agoura Hills, CA 91301, USA, \\ +1-661-803-9939, kenli@optonomous.com \\ 2) Taiwan Color Optics, Inc., 4F., No.32, Keya Rd., Daya Dist., Taichung City, Central Taiwan Science Park, 42881, Taiwan, \\ +886-916-369-588, ypchang@.tcog.com.tw
}

\begin{abstract}
This invited paper presents our current research in the subject of integrated Beam Control Headlight and LiDAR, which includes a DMD pixel-based system and a polygon scanning mirror-based system. Single crystal phosphor is used providing high efficiency and reliability to the system. The goal is to provide highly reliable, compact, and low-cost systems to be introduced into the mass market for autonomous vehicles. In both cases, "hot-spots" can be introduced providing extended ranges and efficiency. The LiDAR system integrates seamlessly with the headlight sharing most of the components, which is the essence of these designs.
\end{abstract}

\section{Introduction}

For mass adoption of the integrated beam control and LiDAR into standard and autonomous vehicles, with the advancement of high-density IC design and fabrication technologies, the cost of optics and related mechanical components become more important. Integration by sharing components becomes an essential directive in the development of such systems. Optonomous Technologies in the United States and Taiwan Color Optics in Taiwan take this approach together in such developments integrating both functions. Single crystal phosphor (SCP) has also been developed and introduced into these integrated structures allowing higher efficiency, higher temperature, and longer life operations.

\section{Laser Excited Single Crystal Phosphor}

SCP provides a highly efficient and reliable light source for automotive applications. To capitalize on the advanced developments of the high brightness LED and the transparent property of the SCP. Figure 1 shows a laser assisted LED where a SCP is placed on top of a white LED with laser excitation from the front. The output of the LED passes through the SCP with the added output from the laser excitation from the front.

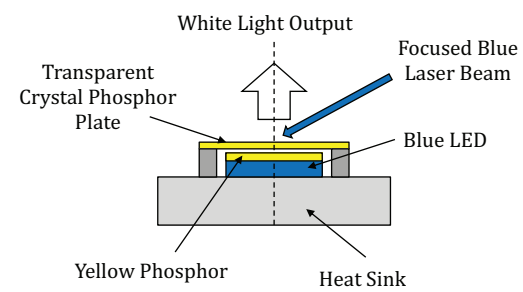

Figure 1 - Structure of a Laser-Assisted LED

\section{Single DMD Headlight with LiDAR}

Figure 2 shows the schematic diagram of a DMD headlight fabricated using a 4,000-lumen LED. The output illumination level has met the standard specifications. In order to provide even high illumination so that other advanced functions, such as extended long- range beam, widened low beam, etc. more light is required for the DMD to be modulated. A novel design of such illumination system is being developed using the laser assisted LED. Over 50\% improvements in output is expected with this laser assisted LED providing new functionality to the DMD headlight.

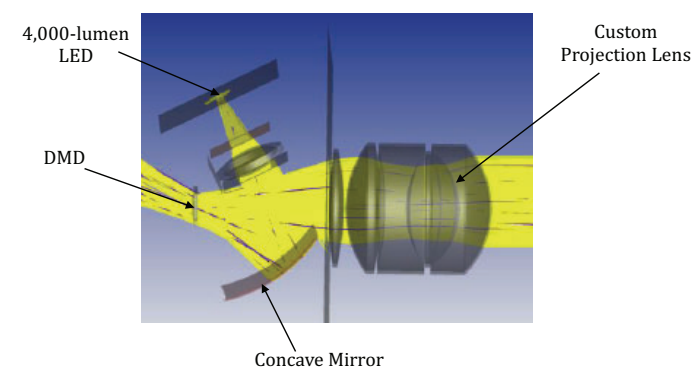

Figure 2 - Schematic Diagram of a DMD Headlight

To further lowering the cost, the LiDAR is integrated into the system using the same DMD as shown in Figure 3. In this design, both the +12 and -12 degrees position of the pixels are used.

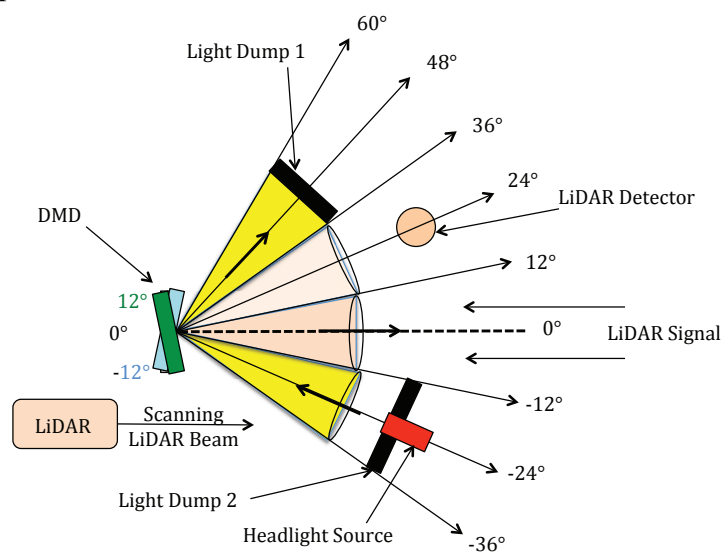

Figure 3 - Single DMD Headlight with Beam Control and LiDAR 


\section{Invited Paper}

As shown in the figure, the LiDAR laser beam is illuminated at the target and the reflected beam is collected at the 0 -deg, which is reflected by the DMD either in the -12-deg or 12-deg positions. At the detection position of $+12-\mathrm{deg}$, the reflected LiDAR signal will be directed to the detector at the 24-deg position. When the DMD mirror is at the -12-deg, the reflected LiDAR signal will be directed to the -24-deg position where the light dump 2 and where the headlight light source are. When the selected position of the DMD, corresponding to the location of the LiDAR beam, is set to have the mirrors switched to the 12-deg position, the reflected signal from the selected location will be directed to the detector for Zdirection determination as described previously.

For the beam control, the headlight source is position at the -24-deg position where the light will be reflected towards the output $(0-\mathrm{deg})$ position towards the roadway when the mirror is at the $-12-\mathrm{deg}$ position. When the mirror is at the 12-deg position, the light will be reflected to the 48-deg direction and absorbed by the light dump 1 . The net effect is that at the selected positions of the LiDAR detection, the headlight will be OFF at these positions and the light will be directed to the light dump 1 (48-deg). For all the un-selected position where the mirrors are at the -12-deg positions, the light will be output to the target as the headlight output beam. Since the selected area is synchronized to the scanning laser beam, the scanning laser beam does not illuminate these un-selected areas and these mirrors could also be switched to 12-deg without affecting the LiDAR detection function.

\section{Single Rotating Polygon with Multi-Level Optics for Integrated Beam Control and LiDAR}

Another integrated structure being developed is a trilevel rotating polygon with one level for beam control, another level for infrared laser scanning beam, and the third level for LiDAR signal detection. The schematic diagram is as shown in Figure 5.

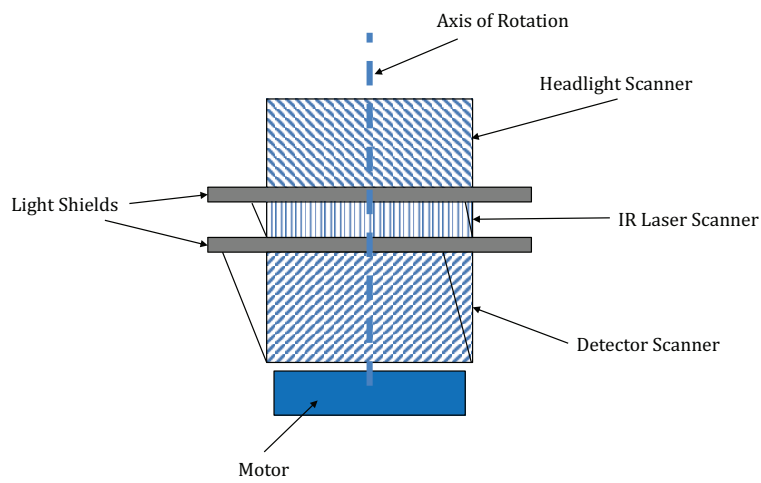

Figure 5 - A Tri-Level Polygon Scanner

At this time, only the headlight beam control section is descried in details. The other sections are being designed. Figure 6 and Figure 7 shows the headlight beam control level in which multiple scan lines can be produced. Each facet of the scanner is designed to produce one scan line and each facet is tilted at a different angle such that the scan lines forms a raster scanned pattern on the target. As shown in the figure, the output scanned lines are focused onto a transmissive phosphor plate, which could be made using SCP or other types of phosphor materials suitable for the output of the system.

The laser diode and the scanning polygon are synchronized such that by modulating the intensity of the laser diode, various patterns on the roadway can be produced by projecting the output of the phosphor plate through the projection lens. The number of scanned lines is the multiple of number of lasers and the number of tilted facets of the polygon. For example, with a system with 4 lasers and 18 tilted polygon mirrors, a total of 72 scanned lines are produced.

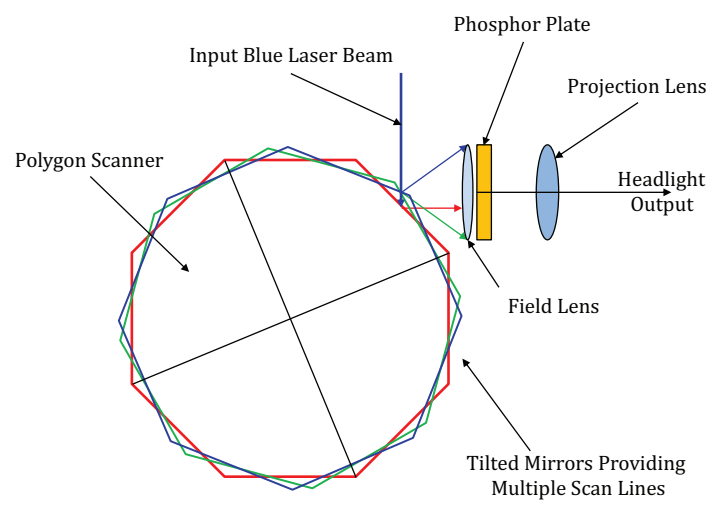

Figure 6 - Headlight Beam Control Polygon Section

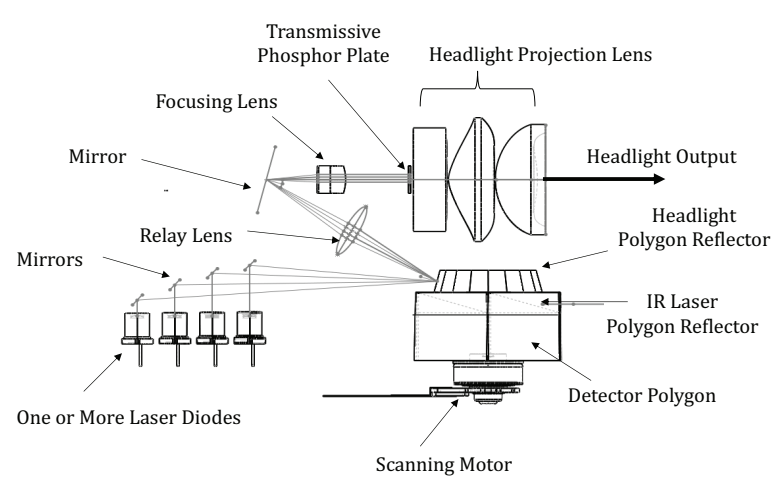

Figure 9 - Tri-Level Polygon Scanner for Integrated Beam Control and LiDAR

\section{Conclusion}

The current research in the subject of integrated Beam Control Headlight and LiDAR, which includes a DMD pixel-based system and a polygon scanning mirror-based system has been presented. Single crystal phosphor is used providing high efficiency and reliability to the system. The goal is to provide highly reliable, compact, and low-cost systems to be introduced into the mass market for autonomous vehicles. 
LDC2021 | Oral Presentation

Laser technology for automotive applications 1

Wed. Apr 21, 2021 2:45 PM - 3:45 PM LDC (Online)

\section{[LDC-7-02] Improvement of Color LiDAR with RGB visible single-mode laser diodes}

OMasato Ishino ${ }^{1}$, Tomoyuki Ohashi ${ }^{1}$, Hiroshi Fuji ${ }^{1}$, Kana Fujioka ${ }^{1}$, Kazuhisa Yamamoto ${ }^{1}$ (1.Osaka University)

We report on the performance improvement of a color LiDAR with RGB visible laser diodes (LD). By using LDs with single lateral mode as the light sources, a short pulse driver, and a high-sensitivity detector, distance and color evaluations with small deviations and a low LD-operation power have been achieved. 


\title{
Improvement of Color LiDAR with RGB visible single-mode laser diodes
}

\author{
Masato Ishino, Tomoyuki Ohashi, Hiroshi Fuji, Kana Fujioka and Kazuhisa Yamamoto, \\ Institute of Laser Engineering, Osaka University, 2-6 Yamada-Oka, Suita Osaka 565-0871 Japan \\ TEL +81-6-6105-5299, email ishino-m@,ile.osaka-u.ac.jp
}

\begin{abstract}
We report on the performance improvement of a color LiDAR with RGB visible laser diodes (LD). By using LDs with single lateral mode as the light sources, a short pulse driver and a high-sensitivity detector, distance and color evaluations with small deviations and a low LD-operation power have been achieved.
\end{abstract}

\section{Introduction}

A color LiDAR (light detecting and ranging), which uses RGB visible light semiconductor lasers, is attractive as an ultra-compact sensor for autonomous driving [1] and autonomous walking of robots [2]. This is because distance measurement and color discrimination can be performed at the same time without a camera or additional lighting, and it can be easily integrated with a lighting and display using the same light sources and scanning systems, which leads to ultra-compact and multipurpose devices [3].

We have proposed and demonstrated a visible LiDAR using LD of three primary RGB colors that can acquire color information and position information simultaneously [4].

This time, we have used single-mode LDs as visible light sources with higher beam quality, to improve the performance.

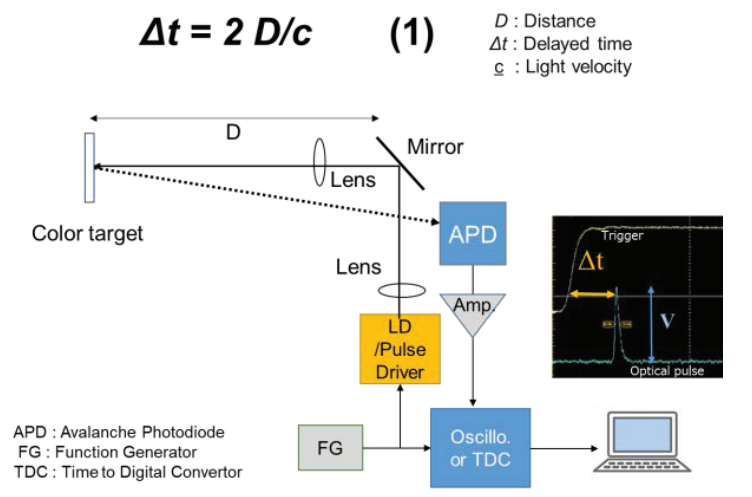

Fig.1 Schematic drawing showing TOF measurement system

\section{Evaluation method}

In order to verify fundamental characteristics of color LiDAR, we performed TOF (Time of Flight) experiment using RGB-LDs color targets with different colors (white, black, yellow, red, green, blue and light blue), as shown in Fig.1. Laser pulses with a width on the order of nanoseconds and a repetition of $10 \mathrm{kHz}$ were illuminated to a color target, and the reflected light from the target was detected by avalanche photodiode (APD). Distance
$D$ is determined by the delayed time $\Delta t$ of the received pulse from the trigger according to eq. (1) in Fig.1, and the target colors were identified by the relative intensities $V$ of received RGB pulses.

To obtain higher beam-quality and safety, we newly used laterally single-mode (SM) lasers as visible light sources of color LiDAR, instead of multi-mode (MM) lasers in the previous work [4]. Major differences in the experimental conditions are listed in Table 1.

Table 1. Comparison of the experimental conditions

\begin{tabular}{|c|c|c|c|}
\hline \multicolumn{2}{|c|}{ Types of LD } & Previous work & This work \\
\hline \multirow{2}{*}{ Optical pulse } & Average power & $500 \mu \mathrm{W}$ & $33 \mu \mathrm{W}$ \\
\cline { 2 - 4 } & Pulse width & $17 \mathrm{~ns}$ & $4 \mathrm{~ns}$ \\
\hline \multirow{3}{*}{ APD } & $\begin{array}{c}\text { Received Area } \\
\text { Diameter }\end{array}$ & $0.2 \mathrm{~mm}$ & $0.5 \mathrm{~mm}$ \\
\cline { 2 - 4 } & $\begin{array}{c}\text { Max. Sensitivity } \\
\text { at Peak }(600 \mathrm{~nm})\end{array}$ & $50 \mathrm{~A} / \mathrm{W}$ & $20 \mathrm{~A} / \mathrm{W}$ \\
\cline { 2 - 4 } & Bandwidth & $400 \mathrm{MHz}$ & $680 \mathrm{MHz}$ \\
\hline
\end{tabular}

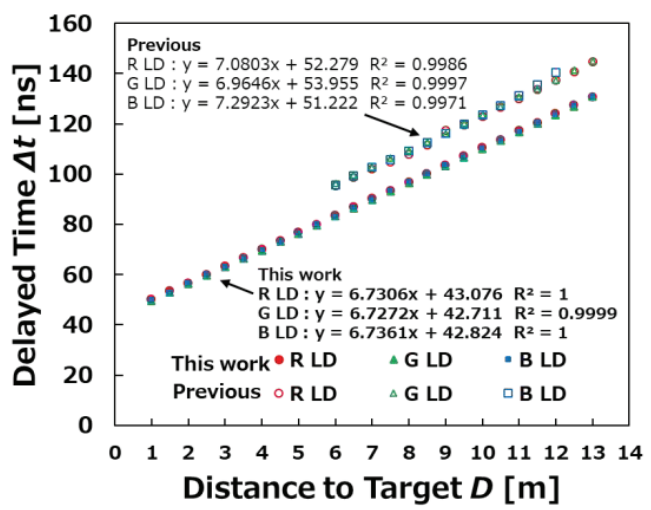

Fig. 2. Delayed time $\Delta t$ of received optical pulse at different distances $D$.

\section{Results and Discussion}

3.1 Distance characteristics

Figure 2 shows the distance dependence of time differences $\Delta \mathrm{t}$ for the RGB LDs used in this work, in comparison with those in the previous work, in which the target color is white. It is found that up to $13 \mathrm{~m}$ each RGB SM-LD can determine distances from the relation 
of $\Delta t$ to $D$ as the same manner as previous results. And a comparison of the coefficient values of determination R2 in both cases shows that the linearity is much higher than before. It is also found that this time the mesureable distance range is expanded to a short distance of $1 \mathrm{~m}$ or less.

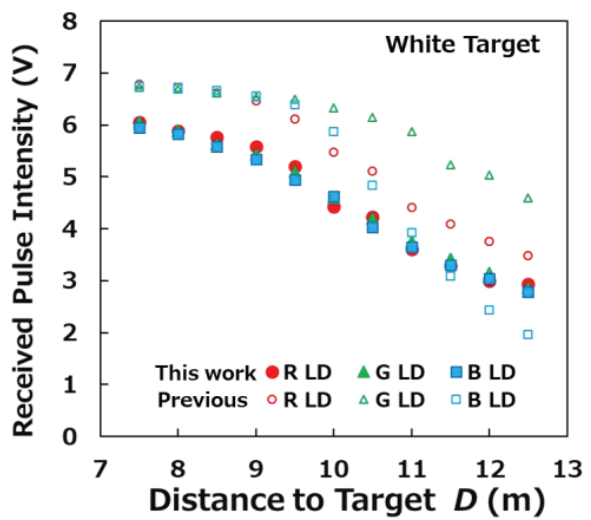

Fig. 3. Received pulse intensities at different distances $D$ for RGB LDs.

Distance characteristics of received pulse intensities for both cases are shown in Fig. 3, in which the target color is white. From the figure, it is confirmed that intensity values in this work are on the same order of those in the previous in spite of its much lower power for LD operation. In addition, while the previous results show that the distance characteristics of reception intensity are much different among the RGB LDs, the results of this work have a highly linear relationship to the distance, and the distance characteristics for the RGB LDs are almost the same.

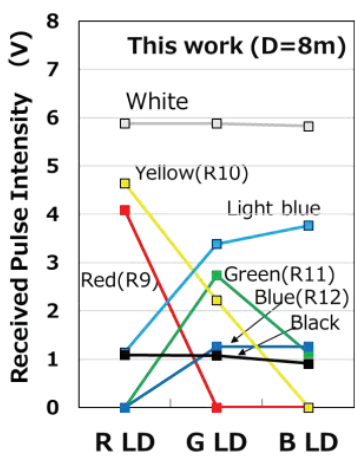

(a)

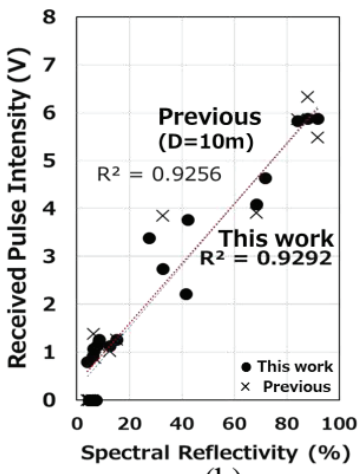

(b)
Fig. 4(a). Received pulse intensity of the RGB LDs for seven color targets at $8 \mathrm{~m}$, (b) relationship of the reception intensity to spectral reflectivity

\subsection{Color discrimination characteristics}

Color discrimination was carried out from the magnitude relationship among the received pulse intensities of RGB LDs for each target. Fig.4(a) shows the received pulse intensities for this work at a distance of $8 \mathrm{~m}$. It is clarified that color identification for each color target is achieved also in the case of SM LDs, from the different intensities among the RGB LDs. Similar characteristics were obtained up to a distance of about $12 \mathrm{~m}$, and it was found that this configuration using SM LD enables color discrimination over a wide distance range. The relationship between the received pulse intensities and the spectral reflectivities for each target at the same wavelengths is shown in Fig. 4(b). It can be seen that the reception intensity and the spectral reflectance in this work have a linear relationship as good as in the previous.

As for the white target, relative intensities of received RGB pulses were converted to chromaticity $\mathrm{x}, \mathrm{y}$, based on CIE1931. The results are shown in Fig.5 for both this work and the previous, which includes the data in the distance range from $8 \mathrm{~m}$ to $12 \mathrm{~m}$. While the previous case has large deviation in $\mathrm{y}$ value among different distances, it becomes quite small in this work. These favorable results are considered due to not only improvement in evaluation methods but also higher beam quality of SM LDs.

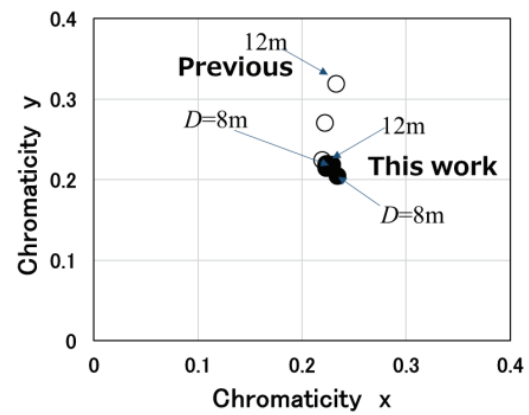

Fig. 5. Calculated chromaticity $\mathrm{x}, \mathrm{y}$ for the white target at different distance.

\section{Conclusion}

Improvement of color LiDAR with RGB visible $\mathrm{LD}$ has been achieved by using higher beam-quality SM LDs, as well as applying advanced operation methods. These results assure us the realization of ultra-compact and high-performance color LiDAR.

\section{Acknowledgment}

The authors express their appreciation to Mr. S. Masui, Nichia Corp. for valuable discussions about characteristics of LDs.

[1] K. Osugi and K. Miyauchi:Proc. Symposium of Advanced Vehicle Control 98 (AVEC '98) 735 (1998)

[2] H. Kawata, A. Ohya, S. Yuta, W. Santosh, and T. Mori: Proc. IEEE/RSJ Int. Conf. on Intelligent Robots and Systems(IROS), 3277 (2005)

[3] M. Ishino, T. Kitamura, A. Takamori, J. Kinoshita, N. Hasegawa, M. Nishikino and K. Yamamoto: Optical Review, 26, 213 (2019)

[4] T.Ohashi, M. Ishino, T. Kitamura, Hiroshi Fuji and Kana Fujioka and K. Yamamoto; The 9th Laser Display and Lighting Conference 2020 (LDC'20), 14-3 
LDC2021 | Oral Presentation

Laser technology for automotive applications 1

Wed. Apr 21, 2021 2:45 PM - 3:45 PM LDC (Online)

\section{[LDC-7-03] Thermal property of AIN-Ce:YAG composite ceramics phosphor for laser lighting}

OTakuya Sawada ${ }^{1}$, Hiroshi Huji ${ }^{1}$, Kenta Yagasaki ${ }^{1}$, Hisashi Minemoto ${ }^{1}$, Yukio Manabe ${ }^{1}$, Kana Fujioka ${ }^{1}$, Kazuhisa Yamamoto ${ }^{1}$ (1.Institute of Laser Engineering, Osaka University)

We have developed AIN-Ce:YAG composite ceramics in order to suppress thermal quenching, and measured thermal property when the ceramics were irradiated with a high-power laser. 


\title{
Thermal property of AIN-Ce:YAG composite ceramic phosphors for laser lighting
}

\author{
Takuya Sawada ${ }^{1}$, Hiroshi Fuji ${ }^{1}$, Kenta Yagasaki ${ }^{1}$, Kana Fujioka ${ }^{1}$, Hisashi Minemoto ${ }^{1}$, Yoshio Manabe ${ }^{1}$ and Kazuhisa Yamamoto ${ }^{1}$ \\ ${ }^{1}$ Institute of Laser Engineering, Osaka University 2-6 Yamadaoka Suita-shi Osaka 565-0871 Japan \\ Phone+81-6-6879-8735, email sawada-t@ile.osaka-u.ac.jp
}

\begin{abstract}
We have proposed composite ceramics in which AlN is added to Ce:YAG ceramics to suppress thermal quenching. The amount offluorescence emitted and the temperature change when the ceramics were irradiated with a high-power laser were measured. The addition of AlN to Ce:YAG is effective for high-power laser lightings.
\end{abstract}

\section{Introduction}

La ser lighting has attracted a s next-generation lighting alternative to LED lighting because of its advantages such as high brightness and high directivity. The advantages make it possible to miniaturize the device and local lighting, so practical application in various fields is expected [1]. However, temperature of a phosphor in a lighting device rises during excita tion with a high-power laser beam, and its fluorescence decreases because of thermal quenching $[2,3]$. We have succeeded in suppressing the thermal quenching of AlN-Ce:YAG composite ceramic phosphors [4]. The phosphors had high thermal conductivity due to AIN grains so that the thermalquenching was suppressed. In this paper, we will evaluate thermal property of the phosphors while irradiating with a high-power blue laser beam, and describe impacts of adding AIN for high power laser lighting.

\section{Experimental procedure}

Ce: YAG composite ceramic phosphors were prepared by a solid-state reaction method. AlN addition ratios to the Ce: YAG were 0, 20, 50 and $80 \mathrm{wt} \%$ [Fig. 1]. The fluorescence intensities of these phosphors were measured using an integrating sphere and a spectrum analyzer. A semiconductor blue la ser with a wa velength of $445 \mathrm{~nm}$ was used for excitation, and its beam spot diameter was $1 \mathrm{~mm}$ and the range of output power was 1 to $10 \mathrm{~W}$. The temperatures at the center of focused laser spots on these phosphors was measured with a thermography camera during the fluorescence [Fig. 2]. The thermal conductivities of these phosphors were estimated by computer simulation using the results of these experiments.

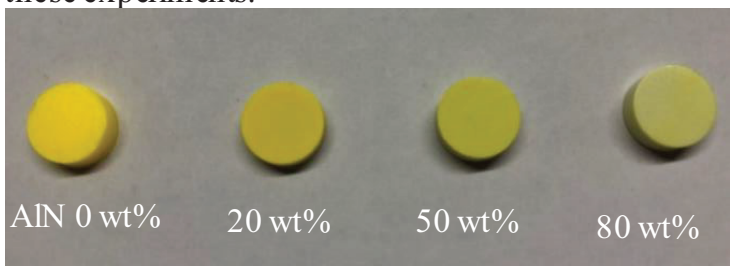

Figure 1 Sample image

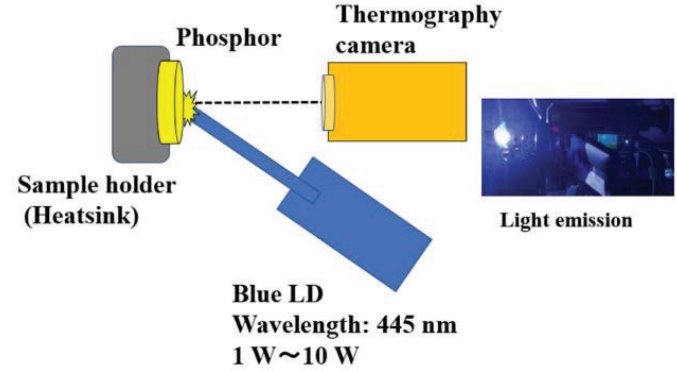

Figure 2 Experimental set up

\section{Results and discussion}

The incident power dependence of the fluorescence intensity is shown in Fig. 3. When the a mount of AIN added was $0 \mathrm{wt} \%$, the fluorescence intensity began to drop sharply at an incident power of $6 \mathrm{~W}$. As the AlN ratio increased, the position of the inflection point shifted to the higher input la ser power of $8.5 \mathrm{~W}$ at the AlN ratio of 20 $\mathrm{wt} \%$. Inflection points at the AlN ratio of $50 \mathrm{wt} \%$ and 80 $\mathrm{wt} \%$ were considered to be $10 \mathrm{~W}$ or more.

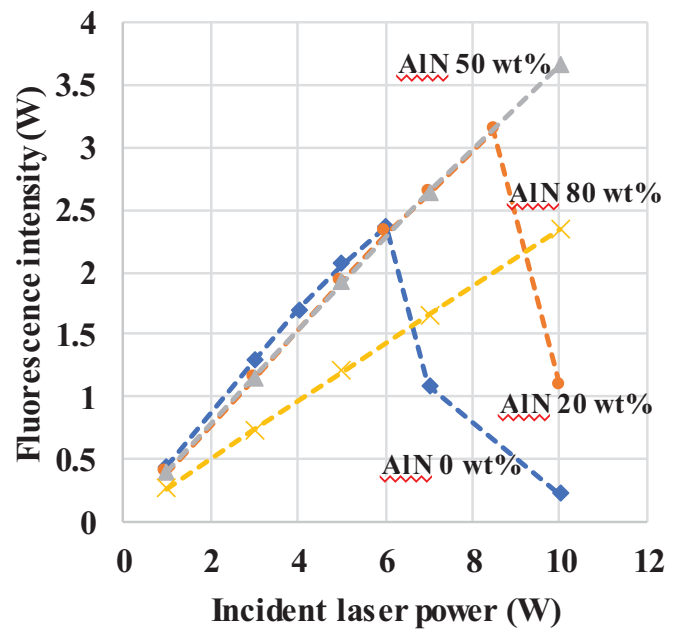

Figure 3 Incident laser power vs fluorescence intensity.

The incident power dependence of the phosphor temperature at the center of focused spot is shown in Fig. 4. At the AlN ratio of $0 \mathrm{wt} \%$, the temperature of the 
phosphor rose sharply at an incident power of $6 \mathrm{~W}$. As the AIN ratio increased to $20 \mathrm{wt} \%$, the boosting point of incident power reached to $8.5 \mathrm{~W}$. The points seemed to be $10 \mathrm{~W}$ or more at the AIN ratios of $50 \mathrm{wt} \%$ and $80 \mathrm{wt} \%$. Comparing with the fluorescence intensity as shown in Fig. 3, the temperature boosted at the inflection points of the fluorescence intensity. Thus, the thermal quenching instantaneously accelerated at these points. It is clarified that the AIN grains improve the thermal conductivity of phosphors so that the a ddition of AlN suppresses themal quenching.

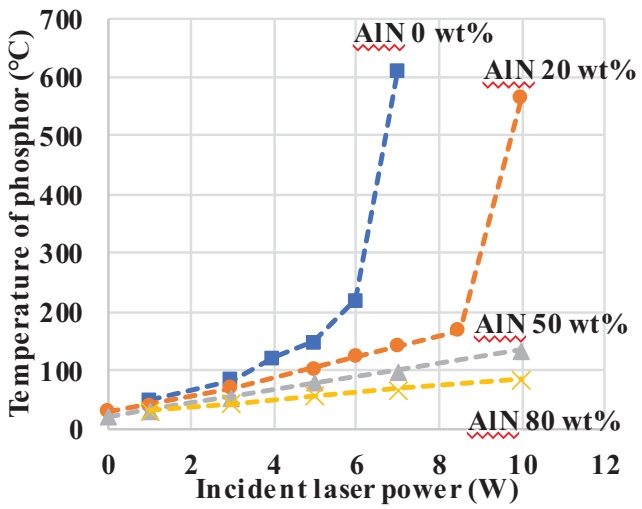

Figure 4 Incident laser power vs temperature of phosphor.

The heat quantity $Q(\mathrm{~W})$ generated in the phosphor can be obtained as the difference between the incident light quantity $I(\mathrm{~W})$ and the luminescent quantity $L(\mathrm{~W})$ of the fluorescence and blue scattered light $(Q=I-L)$. Fig. 5 shows (a) temperature distribution of the phosphor measured by the thermography camera and (b) that calculated by computer simulation. We calculated the thermal conductivity of the disk-shaped phosphors using the heat quantity $Q(\mathrm{~W})$ and the measured temperature $T$ $(\mathrm{K})$ by the simulation. At the center of the focused spot on the phosphors, the thermal conductivity was estimated so that the temperature by the simulation (Fig. 5 (b)) matches the measured temperature (Fig. 5 (a)). The thermal conductivity at $1 \mathrm{~W}$ and $3 \mathrm{~W}$ la ser powers increasedas the AIN ratio increased, similar to that measured by laser flash method as shown in Fig. 6. Although the themal conductivity at the AIN ratio of $80 \mathrm{wt} \%$ is very high, the fluorescence intensity is lower than the others as shown in Fig. 3, so the optimum AIN ratio seems to be $50 \mathrm{wt} \%$ to $80 \mathrm{wt} \%$.
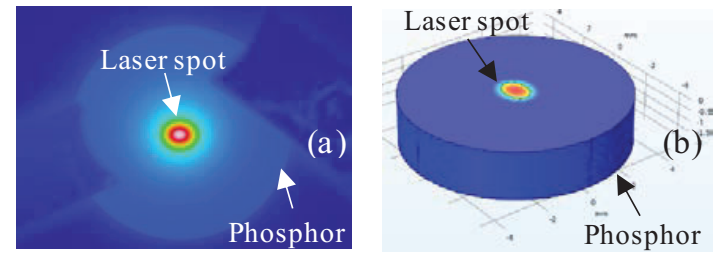

Figure 5 Temperature distribution images by thermography camera (a) and computer simulation (b)

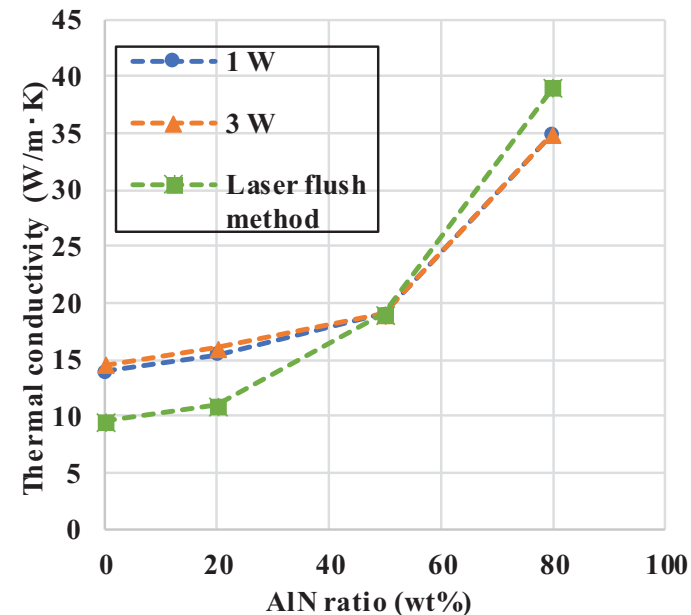

Figure 6 AIN ratio vs thermal conductivity

\section{Conclusion}

Fluorescence emission amount and thermal properties were evaluated experimentally and theoretically. The thermal conductivity of the phosphors increased due tothe addition of AIN, and the amount of fluorescence did not decrea se by suppressing the temperature rise. As a result, it was proved that addition of AlN to Ce:YAG ceramic phosphors was effective for high-power la ser irra diation. In the next step, we will find the optimum ra tio of AIN in order to obta in high heat resistance and high luminous efficiency, and a im to realize high-power la ser lighting.

\section{References}

[1] Jona than J. Wierer, Jr., Jeffrey Y. Tsa o,Dmitry S. SizovJ. "Comparison between blue la sers and lightemitting diodes for future solid-state lighting" Laser Photo. Rev., 7,963-993,(2013).

[2] J. Ueda, Dorenbos, Bos, A. J., Meijerink, A. Ta nabe, $\mathrm{S}$. "Insight into the Thermal Quenching Mechanism for Y3A15O12:Ce3+ Through Thermoluminescence Excitation Spectroscopy." J. Phys. Chem. C., 119, 25003-25008 (2015).

[3] M.R. Krames, O.B. Shchekin, R. Mueller-Mach, G.O. Mueller, L. Zhou, G. Harbers, and M.G. Cra ford: Status and future of high-power light-emitting diodes for solid-sta te lighting. J. Disp. Technol., 3 (2), 160 (2007).

[4] K. Yaga saki et al., "Ce:YAG composite ceramic phosphors for la ser lighting." The 9th Laser Display and Lighting Conference 2020, Yokohama, LDC 11-04. 
LDC2021 | Oral Presentation

\section{Laser technology for automotive applications 2}

Wed. Apr 21, 2021 4:00 PM - 5:00 PM LDC (Online)

Click to the Online Room

[LDC-8-01(Invited)] GaN-based Vertical-Cavity Surface-Emitting Lasers with LatticeMatched AllnN/GaN DBRs

OTetsuya Takeuchi ${ }^{1}$, Satoshi Kamiyama ${ }^{1}$, Motoaki Iwaya ${ }^{1}$, Isamu Akasaki ${ }^{1,2}$

(1.Meijo University, 2.Nagoya University)

4:00 PM - 4:30 PM

[LDC-8-02]

Freeform Lens Design for High-Efficient LED Low-Beam Headlamp Lens

OZhengbo Zhu ${ }^{1}$, Shili Wei ${ }^{1}$, Wenyi $\mathrm{Li}^{1}$, Donglin Ma ${ }^{1}$ (1. Huazhong University of Science and Technology)

4:30 PM - 4:45 PM

[LDC-8-03]

High Power Laser Phosphor Light Source using Tilted Rotating Mirror

OKenneth $\mathrm{Li}^{1}$, Y. P. Chang ${ }^{2}$, Lion Wang ${ }^{2}$, Andy Chen ${ }^{2}$, Stark Tsai ${ }^{2}$ (1.Optonomous Technologies Inc., 2.Taiwan Color Optics, Inc.)

4:45 PM - 5:00 PM 
LDC2021 | Oral Presentation

Laser technology for automotive applications 2

Wed. Apr 21, 2021 4:00 PM - 5:00 PM LDC (Online)

\section{[LDC-8-01(Invited)] GaN-based Vertical-Cavity Surface-Emitting Lasers with Lattice-Matched AllnN/GaN DBRs}

OTetsuya Takeuchi ${ }^{1}$, Satoshi Kamiyama ${ }^{1}$, Motoaki Iwaya ${ }^{1}$, Isamu Akasaki ${ }^{1,2}$ (1.Meijo University, 2.Nagoya University)

GaN-based vertical-cavity surface-emitting lasers (VCSELs) have been intensively developed, showing high performances toward practical uses. We show in-situ wafer curvature evolutions during the epitaxial growth of AllnN/GaN DBRs and an implementation of nano-height cylindrical waveguides in the VCSELs. Roomtemperature continuous-wave operations of the VCSELs with large apertures up to $30 \mathrm{~m}$ diameter have been demonstrated. 


\title{
GaN-based Vertical-Cavity Surface-Emitting Lasers with Lattice-Matched AIInN/GaN DBRs
}

\author{
T. Takeuchi ${ }^{1}$, S. Kamiyama ${ }^{1}$, M. Iwaya ${ }^{1}$, I. Akasaki ${ }^{1,2}$ \\ ${ }^{1}$ Department of Materials Science and Engineering, Meijo University, 1-501 Shiogamaguchi, Tempaku-ku, Nagoya 468-8502, Japan \\ Phone: +81-52-838-2293, email: take@meijo-u.ac.jp \\ ${ }^{2}$ Akasaki Research Center, Nagoya University, Furo-cho, Chikusa-ku, Nagoya 464-8601, Japan
}

\begin{abstract}
GaN-based vertical-cavity surface-emitting lasers (VCSELs) have been intensively developed, showing high performances toward practical uses. We show in-situ wafer curvature evolutions during the epitaxial growth of AlInN/GaN DBRs and an implementation of nano-height cylindrical waveguides in the VCSELs. Room-temperature continuous-wave operations of the VCSELs with large apertures up to $30 \mu$ m diameter have been demonstrated.
\end{abstract}

\section{Introduction}

Visible GaN-based vertical-cavity surface-emitting lasers (VCSELs) have been intensively developed toward novel light sources for various applications, such as retinal scanning displays, adaptive headlights, highspeed visible light communication systems, and medical biosensors for Point-of-Care Testing. In principle, VCSELs require three key structures, such as an active region, an optical/current confinement, and a cavity with a pair of distributed Bragg reflectors (DBRs). Especially for the optical/current confinements and the DBRs in the GaN-based VCSELs, various approaches have been attempted so far.

Cosendey et al. proposed to utilize AlInN/GaN DBRs as the bottom DBRs, showing room temperature (RT) pulsed operations of the GaN-based VCSELs with the DBRs [1]. We then achieved RT continuous-wave (CW) operations of GaN-based VCSELs with n-type conducting AlInN/GaN DBRs [2]. In any case, the AlInN layers must be lattice-matched to GaN, requiring a precise composition control of AlInN. Krost et al. demonstrated that in-situ curvature measurements provide information on strain conditions in the AlInN/GaN DBR during the growth [3]. At the same time, measured and calculated in-situ curvature evolutions of the DBRs were not well-matched. Regarding the optical/current confinement, Kuramoto et al. proposed a nano-height cylindrical waveguide (NCW), showing over $20 \mathrm{~mW}$ light output powers from the VCSEL [4]. A single etching process was sufficient to form the optical/current confinement structure, but the details were not reported yet.

We have developed a practical model for the in-situ curvature evolutions of the AlInN/GaN DBRs with not
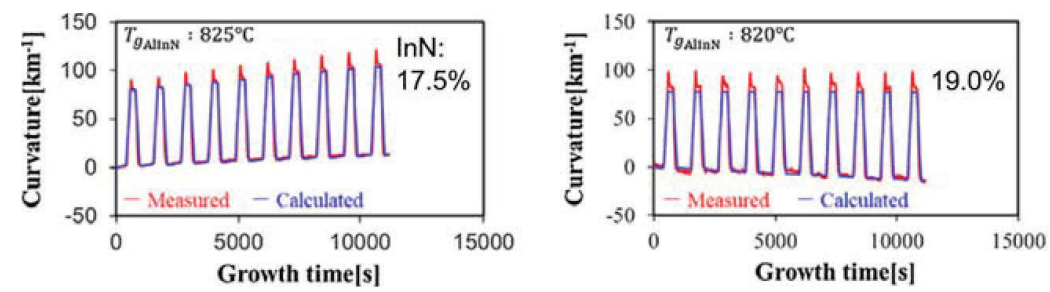

only Stoney formula but also experimental data, providing well-fitted simulated evolution profiles and consistent InN mole fractions [5]. We also have developed the NCW structures with an use of a appropriate etching gas, $\mathrm{BCl}_{3}$, resulting in the VCSEL operations up to $30 \mu \mathrm{m}$ aperture diameter [6].

\section{In-situ curvature evolutions of AIInN/GaN DBRs}

We have equipped EpiCurve TT (Laytec) on top of our MOVPE reactor to obtain in-situ wafer curvature values during the epitaxial growth. We first measured in-situ curvature evolutions during the $25 \mathrm{GaN}$ layers $(55 \mathrm{~nm})$ on a $\mathrm{GaN}$ template/sapphire. The growth sequence was the same as our AlInN $\left(875^{\circ} \mathrm{C}\right) / \mathrm{GaN}\left(1035^{\circ} \mathrm{C}\right) \mathrm{DBR}$ with the temperature ramping steps but no supply of the $\mathrm{Al}$ and In sources, so that we could obtain experimental data of the temperature ramping up/down steps and the $\mathrm{GaN}$ growth step in the sequence. We then found that a top part of the evolution corresponding to the $\mathrm{GaN}$ growth was flat, suggesting that the strain in the GaN layers was zero. At the same time, a bottom part of the evolution linearly went down as the growth progressed. The value was increased downwards by $13 \mathrm{~km}^{-1}$ from 80 $\mathrm{km}^{-1}$ at the beginning to $93 \mathrm{~km}^{-1}$ at the end. The increase $\left(13 \mathrm{~km}^{-1}\right)$ should come from the increase of the epitaxial layer thickness. The remaining step, the AlInN growth step, was simply described by Stoney formula with a fitting parameter of the InN mole fraction, so that we developed a practical model for the in-situ curvature evolutions of the entire AlInN/GaN DBRs.

Figure 1 shows measured and simulated in-situ curvature evolutions during the $\mathrm{AlInN} / \mathrm{GaN}$ growth with the growth temperatures of $825,820,815^{\circ} \mathrm{C}$. The simulated evolutions with our model were in excellent

Fig. 1 measured and simulated in-situ curvature evolutions of three AlInN/GaN DBRs [5]. 
agreement with the measured ones, providing the $\mathrm{InN}$ mole fractions which were consistent with the ones from ex-situ XRD measurements. The in-situ curvature monitoring is a powerful tool for the growth of highquality AlInN/GaN DBRs.

\section{Nano-height cylindrical waveguide structure}

The NCW structure basically consist of a 5nm-high mesa of $\mathrm{p}-\mathrm{GaN}$ contact layer for the optical confinement, formed by a single etching step. The etching step simultaneously formed highly resistive etched surface, resulting in a function of the current confinement. We first investigated etching gas dependences, such as $\mathrm{BCl}_{3}$, $\mathrm{O}_{2}$, and $\mathrm{Ar}$, on the contact resistance with the etched surfaces. We found that the $\mathrm{BCl}_{3}$ case showed the highest p-contact resistance, suggesting the best choice in terms of the current confinement.

Next, we implemented the $5 \mathrm{~nm}$-high NCW structures in GaN-based VCSELs emitting a $413 \mathrm{~nm}$ wavelength, formed by $\mathrm{BCl}_{3}$ etching. The diameters of the $\mathrm{NCW}$ structures were 5 to $30 \mu \mathrm{m}$. Figure 2 shows I-L-V characteristics of the GaN-based NCW VCSELs with the 5-30 $\mu \mathrm{m}$ diameters. RT CW operations were obtained from all the NCW VCSELs. As far as we know, the reported largest aperture of GaN-based VCSELs was 20 $\mu \mathrm{m}$, thus the $30 \mu \mathrm{m}$ aperture in this work is one of the largest ones. At the same time, the maximum light output powers (LOPs) were less than $4 \mathrm{~mW}$ regardless of the aperture sizes. We concluded that the maximum LOPs were limited by device temperatures from temperature increases estimated with simple analytical device thermal resistance values [7].

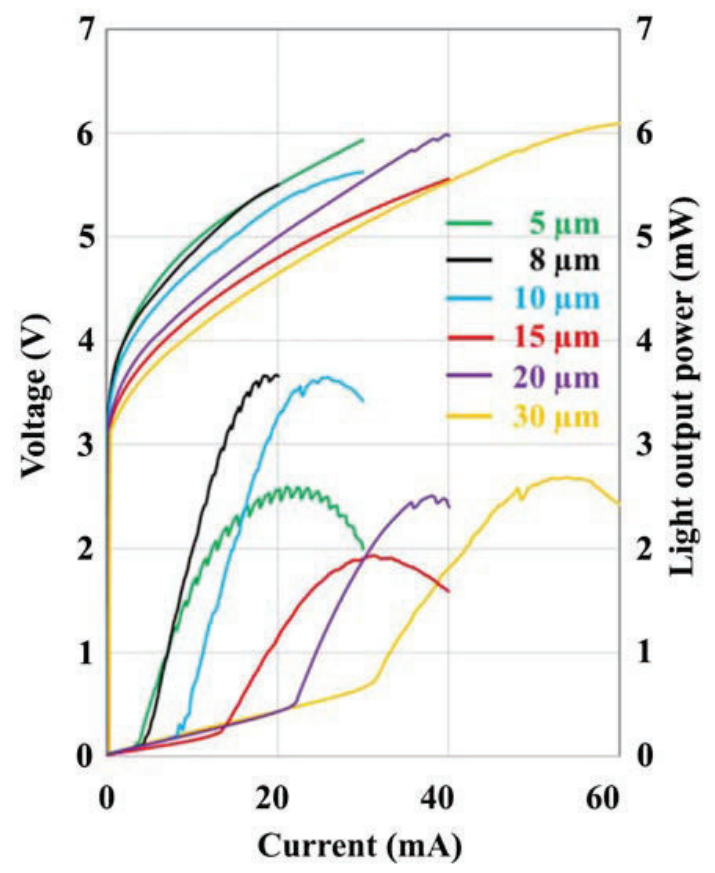

Fig. 2 I-L-V characteristics of GaN-based NCW VCSELs with 5-30 $\mu \mathrm{m}$ diameters [6].

\section{Conclusion}

We have developed the practical model of the in-situ curvature evolutions of the AlInN/GaN DBRs, providing well-matching profiles with measured ones and determinations of consistent $\mathrm{InN}$ mole fractions. We also achieved the GaN-based NCW VCSELs with large apertures up to $30 \mu \mathrm{m}$ diameter, but resulting in a limitation of LOPs less than $4 \mathrm{~mW}$.

\section{Acknowledgment}

This study was partly supported by MEXT "Program for research and development of next-generation semiconductor to realize energy-saving society", MEXT Private University Research Branding Project (20162020), JSPS KAKENHI for Scientific Research A [20H00353], and JSPS KAKENHI for Innovative Areas [16H06416], and JST CREST [16815710].

\section{References}

[1] Gatien Cosendey, Antonino Castiglia, Georg Rossbach, Jean-Francois Carlin, and Nicolas Grandjean, "Blue monolithic AlInN-based vertical cavity surface emitting laser diode on free-standing GaN substrate", Appl. Phys. Lett. 101, 151113 (2006).

[2] Kazuki Ikeyama, Yugo Kozuka, Kenjo Matsui, Shotaro Yoshida, Takanobu Akagi, Yasuto Akatsuka, Norikatsu Koide, Tetsuya Takeuchi, Satoshi Kamiyama, Motoaki Iwaya, and Isamu Akasaki, "Room-temperature continuous-wave operation of GaN-based vertical-cavity surface-emitting lasers with n-type conducting AlInN/GaN distributed Bragg reflectors ", Appl. Physi. Express 9, 102101 (2016).

[3] A. Krost, C. Berger, J. Bläsing, A. Franke, T. Hempel, A. Dadgar, and J. Christen, "Strain evaluation in AlInN/GaN Bragg mirrors by in situ curvature measurements and ex situ x-ray grazing incidence and transmission scattering", Appl. Phys. Lett. 97, 181105 (2010).

[4] Masaru Kuramoto, Seiichiro Kobayashi, Takanobu Akagi, Komei Tazawa, Hiroki Tanaka, and Tetsuya Takeuchi, "Nano-height cylindrical waveguide in GaN-based verticalcavity surface-emitting lasers", Appl. Phys. Express 13, 082005 (2020)

[5] Kei Hiraiwa, Wataru Muranaga, Sho Iwayama, Tetsuya Takeuchi, Satoshi Kamiyama, Motoaki Iwaya, Isamu Akasaki, "In-situ curvature measurements of AlInN/GaN distributed Bragg reflectors during growths containing substrate temperature ramping steps", J. Crys. Growth 531, 125357 (2020)

[6] Ryosuke Iida, Yusuke Ueshima, Sho Iwayama, Tetsuya Takeuchi, Satoshi Kamiyama, Motoaki Iwaya, Isamu Akasaki, Masaru Kuramoto, and Toshihiro Kamei, "Aperture diameter dependences in GaN-based verticalcavity surface-emitting lasers with nano-height cylindrical waveguide formed by $\mathrm{BCl} 3$ dry etching", Appl. Phys. Express 14, 012003 (2021).

[7] L. A. Coldren and S. W. Corzine, "Diode Lasers and Photonic Integrated Circuits", (Wiley, New York, 1955), p. 57. 
LDC2021 | Oral Presentation

Laser technology for automotive applications 2

Wed. Apr 21, 2021 4:00 PM - 5:00 PM LDC (Online)

\section{[LDC-8-02] Freeform Lens Design for High-Efficient LED Low-Beam Headlamp Lens}

OZhengbo Zhu' ${ }^{1}$, Shili Wei ${ }^{1}$, Wenyi $\mathrm{Li}^{1}$, Donglin $\mathrm{Ma}^{1} \quad$ (1.Huazhong University of Science and Technology)

We develop a least-squares ray mapping method to design freeform lens for LED-based low-beam headlamp optical system, the produced irradiance distribution conformed to the United Nations Economic Commission for Europe vehicle regulations (ECE) R112. The energy efficiency reaches up to $84 \%$ considering the Fresnel loss. 


\title{
Freeform Lens Design for High-Efficient LED Low-Beam Headlamp Lens
}

\author{
Zhengbo Zhu ${ }^{1}$, Shili Wei ${ }^{1}$, Wenyi Li ${ }^{1}$, Donglin Ma ${ }^{1, *}$ \\ ${ }^{1}$ School of Optical and Electronic Information and Wuhan National Laboratory for Optoelectronics, Huazhong University of Science \\ and Technology, Wuhan, Hubei 430074, China \\ * Corresponding author: madonglin@hust.edu.cn
}

Abstract: We develop a least-squares ray mapping method to design freeform lens for LED-based low-beam headlamp optical system, the produced irradiance distribution conformed to the United Nations Economic Commission for Europe vehicle regulations (ECE) R112. The energy efficiency reaches up to $84 \%$ considering the Fresnel loss.

\section{Introduction}

Lighting quality is essential at night or in dark places when driving. Good visibility can effectively reduce the occurrence of traffic accidents. For the low-beam headlamp lighting system, it not only required to provide enough roadway illuminance but also should be endowed with anti-glare function[1]. Realizing highly efficient low-beam headlamp while maintaining a sharp $15^{\circ}$ inclined cut-off line is an important issue in the automobile industry. The traditional projective low-beam headlamps generally use a baffler to produce a sharp horizontal and $15^{\circ}$ inclined cut-off line on the vertical measuring screen $[2,3]$. While the energy efficiency is usually low $(<50 \%)$ due to the substantial light rays being blocked by the baffle. It is a trend that more and more automobile brands have been adopting LED sources in their new models due to the incomparable advantages of LED light source. In this work, we developed a least-squares ray mapping method to design freeform lens for LED low-beam headlamp optical system to meet the ECE R112 regulation[4].

\section{Design method}

Considering the specific illumination requirement of low-beam headlamp, we choose three effective lighting areas to form the prescribed illumination pattern. As shown in Fig. 1, the measuring screen is divided into four zones: zone $\mathrm{A}$, zone $\mathrm{B}$, zone $\mathrm{C}$ and zone $\mathrm{D}$. Referring to the regulation, especially for the checking points, we find that when the irradiance ratio of the first three zones being set as $E_{\mathrm{A}}: E_{\mathrm{B}}: E_{\mathrm{C}}=8: 4: 3$, the irradiance distribution on the measuring screen can satisfy the ECE R112 regulation well. Zone D is a dark region, and the irradiance value of this region is set zero.

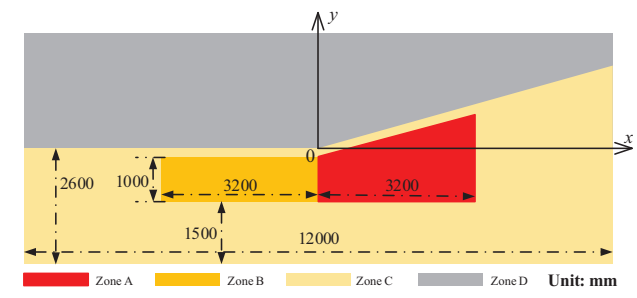

Fig. 1. Prescribed irradiance on the measuring screen.
For the freeform lens design, we use the ray mapping method to calculate the freeform surface. The aim of this method is to find a source-target map $\mathbf{m}$ to redistributing the source energy density $f$ to the target energy density $g$ while maintaining the energy conservation and boundary problems[5], it can be formulated as:

$$
\left\{\begin{array}{l}
g(\mathbf{m}(u, v)) \operatorname{det}(D \mathbf{m}(u, v))=f(u, v), \\
\mathbf{m}\left(\partial \Omega_{0}\right)=\partial \Omega_{1},
\end{array}\right.
$$

where $D \mathbf{m}$ is the Jacobi matrix of $\mathbf{m}, \Omega_{0}$ and $\Omega_{1}$ represent the source and target domain respectively, $\partial \Omega$ stands for the boundary of domain $\Omega$.

It is also important to make the calculated ray mapping provide an integrable surface normal vector fields[6]:

$$
\mathbf{N} \cdot(\nabla \times \mathbf{N})=0
$$

where $\mathbf{N}$ denotes the surface normal vector field.

In this work, we developed a least-squares ray mapping method to calculate the source-to-target mapping satisfying Eqs. (1)-(2). The calculation procedure is implemented by solving three minimization problems to iteratively correct an integrable real map to approach energy conservation and boundary problems[7]. The whole process of calculation of ray mapping is to find an integrable source-target map that generates the continuous freeform surfaces that provide the desired illuminance distribution.

After the source-target map being obtained, we construct the freeform surface according to the calculated ray mapping through the point-by-point freeform surface construction method [8].

\section{Design result}

The obtained freeform lens is illustrated in Fig. 2(c), the size of the lens is restricted to $90 \mathrm{~mm} * 92 \mathrm{~mm}$ (largest diameter $*$ height). The material of the freeform lens is polymethyl methacrylate (PMMA) and its refractive index is about 1.493 . We choose the OSRAM Headlamp Pro LED UW U1A2 01 as the light source [9], and the flux of the LED source is set as $300 \mathrm{~lm}$. After the MonteCarlo ray tracing simulation, we obtained the illumination patterns on the measuring screens as shown 
in Fig. 2(a, b). The vertical measuring screen and the tilted measuring screen with $5^{\circ}$ to the horizontal which are placed 25 meters in front of the low-beam headlamp. The tilted measuring screen set here is to check its irradiance distribution on the roadway surface. The energy efficiency reaches up to $84 \%$ (Fresnel loss is considered).

The comparison of simulated illumination and required illumination in lux for the ECE R112 regulation is listed in Table 1, which indicates that the optical performance of the designed low-beam lens can fully comply with the regulation.

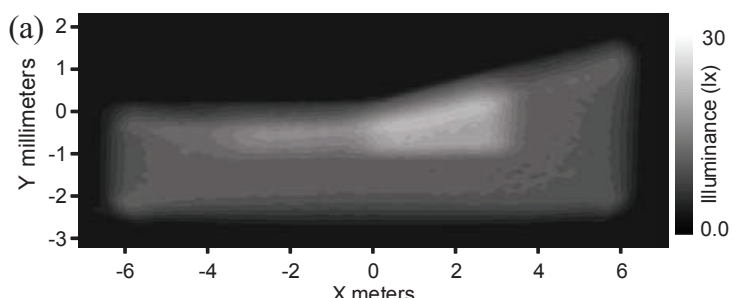

(b)

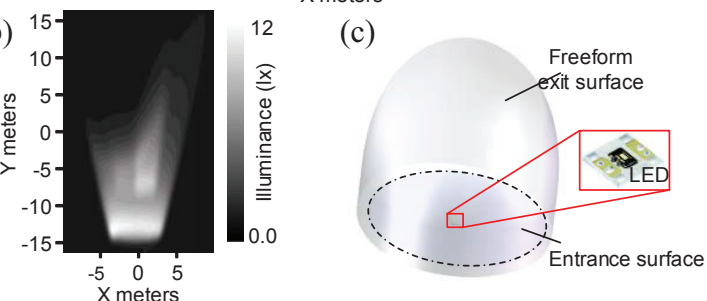

Fig. 2. Illumination patterns on (a) the vertical measuring screen and (b) the titled measuring screen, (c) the designed freeform lens.

Table. 1. Illuminance values of the checking points.

\begin{tabular}{c|c|c|c|c}
\hline Checking points & $75 \mathrm{~V}$ & $75 \mathrm{R}$ & $50 \mathrm{~L}$ & $50 \mathrm{R}$ \\
\hline $\begin{array}{c}\text { Required } \\
\text { illuminance (lux) }\end{array}$ & $\leqslant 12$ & $\geqslant 12$ & $\leqslant 15$ & $\geqslant 12$ \\
\hline $\begin{array}{c}\text { Simulated } \\
\text { illuminance (lux) }\end{array}$ & 8.6 & 18.1 & 7.5 & 19.2 \\
\hline \multicolumn{4}{c}{} \\
\hline Checking points & $50 \mathrm{~V}$ & $25 \mathrm{~L}$ & $25 \mathrm{R}$ & $\mathrm{B} 50 \mathrm{~L}$ \\
\hline $\begin{array}{c}\text { Required } \\
\text { illuminance (lux) }\end{array}$ & $\geqslant 6$ & $\geqslant 2$ & $\geqslant 2$ & $\leqslant 0.4$ \\
\hline
\end{tabular}

\begin{tabular}{c|c|c|c|c}
\hline $\begin{array}{c}\text { Simulated } \\
\text { illuminance (lux) }\end{array}$ & 14.3 & 7.6 & 6.3 & 0.1 \\
\hline
\end{tabular}

\section{Conclusion and outlook}

What we have done above is a brief introduction to LED low-beam headlamp lens design based on the leastsquares ray mapping method. Future work may include the investigation of the optimization process when the larger size LED is used, as well as other freeform surface technology-based illumination applications.

\section{Acknowledgment}

This work was supported by National Natural Science Foundation of China (61805088); Science, Technology, and Innovation Commission of Shenzhen Municipality (JCYJ20190809100811375); Innovation Fund of WNLO.

\section{References}

[1] J.F.V. Derlofske, M.W. Mccolgan, White led sources for vehicle forward lighting, Proc. SPIE 4776 (2002).

[2] C. Hsieh, Y. Li, and C. Hung, "Modular design of the LED vehicle projector headlamp system," Appl. Opt. 52(21), 5221-5229 (2013)

[3] Ge, W. Wang, Z. Du, P. Qiu, J. Wang, and J. Cai, "Highenergy-efficiency optical system for an LED-based headlamp architecture," Appl. Opt. 52(34), 8318-8323 (2013)

[4] United Nations Economic Commission for Europe vehicle regulations, Reg. 112-Rev. 2.

[5] C. Prins, R. Beltman, J. ten Thije Boonkkamp, W. IJzerman, and T. W. Tukker, "A least-squares method for optimal transport using the monge-ampère equation," SIAM J. Sci. Comput. 37(6), B937-B961 (2015).

[6] F. R. Fournier, W. J. Cassarly, and J. P. Rolland, "Fast freeform reflector generation using source-target maps," Opt. Express 18(5), 5295-5304 (2010).

[7] S. Wei, Z. Zhu, Z. Fan, and D. Ma, "Least-squares ray mapping method for freeform illumination optics design," Opt. Express 28(3), 3811-3822 (2020)

[8] Z. Feng, L. Huang, G. Jin, and M. Gong, "Designing double freeform optical surfaces for controlling both irradiance and wavefront," Opt. Express 21(23), 2869328701 (2013).

[9] https://www.osram.com.cn/os/products/index.jsp. 
LDC2021 | Oral Presentation

Laser technology for automotive applications 2

Wed. Apr 21, 2021 4:00 PM - 5:00 PM LDC (Online)

\section{[LDC-8-03] High Power Laser Phosphor Light Source using Tilted} Rotating Mirror

OKenneth $\mathrm{Li}^{1}$, Y. P. Chang ${ }^{2}$, Lion Wang ${ }^{2}$, Andy Chen ${ }^{2}$, Stark Tsai ${ }^{2}$ (1.Optonomous Technologies Inc., 2.Taiwan Color Optics, Inc.)

This paper describes a stationary phosphor plate system excited by a scanning laser focused spot on the phosphor increasing the excitation area, while keeping the same etendue. Such scanning is created by using a tilted rotating mirror reflecting the input laser beam towards the phosphor plate and reflecting the output beam towards the direction of the input laser beam maintaining the small etendue of a single focus spot. 


\title{
High Power Laser Phosphor Light Source using Tilted Rotating Mirror
}

\author{
Kenneth $\mathrm{Li}^{1)}$, Yung Peng Chang ${ }^{2)}$, Lion Wang ${ }^{2)}$, Andy Chen ${ }^{2)}$, Stark Tsai ${ }^{2)}$ \\ 1) Optonomous Technologies Inc., 30330 Rainbow View Ct., Agoura Hills, CA 91301, USA, \\ +1-661-803-9939, kenli@optonomous.com \\ 2) Taiwan Color Optics, Inc., 4F., No.32, Keya Rd., Daya Dist., Taichung City, Central Taiwan Science Park, 42881, Taiwan, \\ +886-916-369-588, ypchang@,tcog.com.tw
}

\begin{abstract}
This paper describes a stationary phosphor plate system excited by a scanning laser focused spot on the phosphor effectively increasing the excitation area, reducing the power density facilitating heat sinking and avoiding saturation. Such scanning is created by using a tilted rotating mirror reflecting the input laser beam towards the phosphor plate and reflecting the output beam towards the direction of the input laser beam maintaining the small etendue of a single focus spot. The resulting high output power can be used for cinema projectors and other high-power applications.
\end{abstract}

\section{Introduction}

The efficiency of blue laser has been improved continuous over the last few years with lowering cost. As a result, laser excited phosphor become an important subject for illumination and lighting applications. Laser diode arrays are getting popular in various light system from low power to very high-power digital projection systems. Cooling and power saturation of the phosphor. This paper presents a stationary phosphor system in which the phosphor plate is not in motion and can be attached to heat sinks for efficient removal of heat at high power operations. A tilted rotating mirror is used to scan the focused laser beam onto the stationary phosphor plate such that the total scanned area is effectively increased, lowering both the optical power density and thermal density. This allows an increase in the overall optical output of the system, eventually, higher projected screen brightness.

\section{Locus of the Focused Laser Beam}

Figure 1 shows the picture of the phosphor plate used for this experiment on which the focused blue laser locus follows.

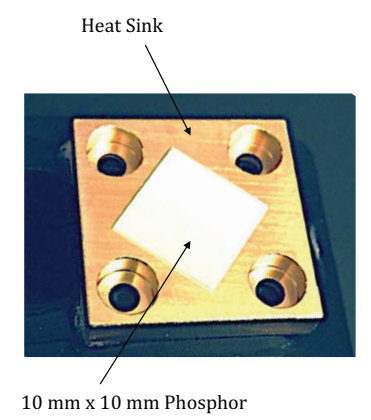

Figure 1 - The Phosphor Plate

Figure 2 shows an example where a $2 \mathrm{~mm}$ spot size at the focus is moving in a circular path with an outer diameter of $6 \mathrm{~mm}$. The effective area increases from $3.14 \mathrm{sq}$. $\mathrm{mm}$. to 25.1 sq. mm., which is 8 times the area of the focused spot, thus increasing the effective area and saturation threshold by 8 times. The key to the design and operation of this system is to maintain the original etendue of the single focus spot with area of 3.14 sq. $\mathrm{mm}$.

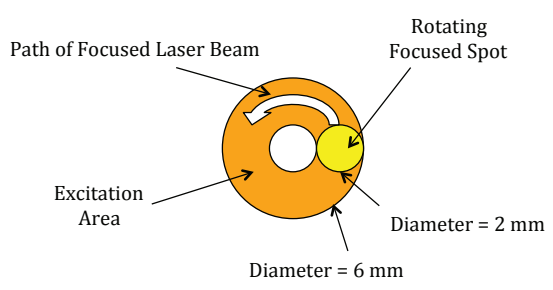

Figure 1 - Locus of the Focus Laser Spot on the Phosphor Plate

\section{Tilted Rotating Mirror Laser Phosphor System} Figure 2 shows the schematic diagram of a tilted rotating mirror. As the motor is running, the axis of the mirror will form a cone with the angle determined by the tilt angle. When a beam of light is reflected by this tilted mirror, it will also for a cone of light with the cone angle being twice the tilt angle.

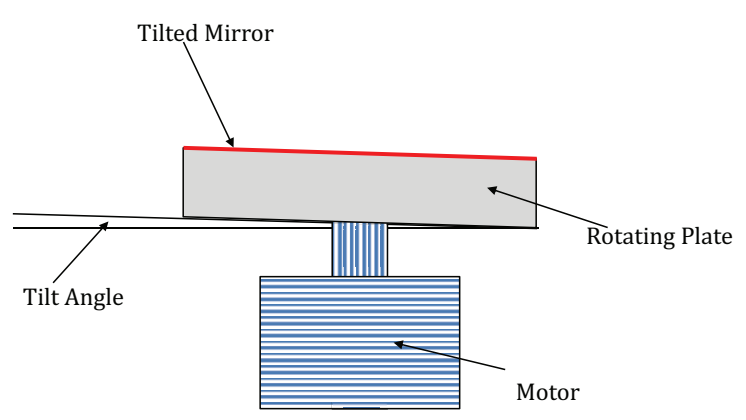

Figure 2 - A Tilted Rotating Mirror

Figure 3 shows the schematic diagram of the system with the tilted rotating mirror. The collimated excitation blue laser beam is reflected by the mirror and focused by the collimating lens onto the phosphor plate, which is a circle as shown in Figure 2. The output white light from 
the phosphor plate, which is Lambertian, will be collected by collimating lens becoming a white parallel beam propagating in the opposite direction of the blue laser beam with the same optical axis, reflected by the tilted mirror and outputs in the same direction as the input laser beam, also with the same optical axis regardless what position the tilted mirror is.. Since the output beam is stationary, the etendue of the output will be the same as if the mirror is not rotating with a stationary focused spot, i.e. not increased due to the rotation. This is an important feature as the coupling efficiency is dependent on the etendue. Increasing power with increase etendue does not increase the output of the projector.

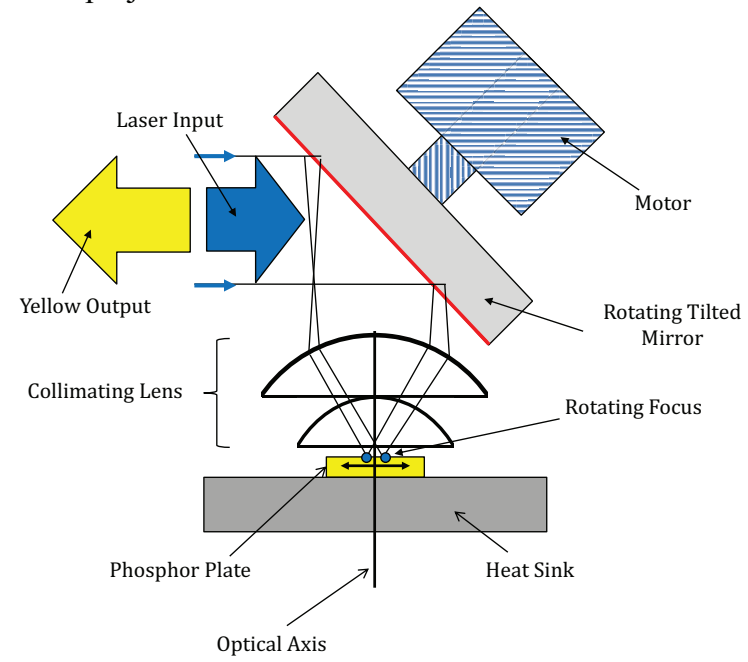

Figure 3 - Schematic Diagram of the Tilted Rotation Laser Phosphor system

\section{Experimental Results}

Initial measurements have been made using a system as shown in Figure 3 with a blue light filter place at the input such that the output can be directed toward the integrating sphere. A two-dimensional laser array from Nichia is used which is focused into a small spot size of $0.48 \mathrm{~mm}$ with an area of $0.18 \mathrm{sq}$. $\mathrm{mm}$ onto the phosphor plate. When the mirror is rotating, it generates a total scanned area of 1.36 sq. mm, which is 7.5 times the single focused spot area. As shown in Figure 4, a total laser power of $54.6 \mathrm{~W}$ is reached. This equates to a equivalent power density of $303 \mathrm{~W} / \mathrm{sq}$. mm. Based on the specified power limitation at about $45 \mathrm{~W} / \mathrm{sq}$. mm., this rotating mirror system increased the power handling limitation by over 6.7 times. Further experiments are being perform increasing the heat sinking capacity of the phosphor plate. For even higher power up to $1,000 \mathrm{~W}$ of laser output with a realistic spot size of $2 \mathrm{~mm}$, and a power density limit of $50 \mathrm{~W} / \mathrm{sq}$. mm, an efficiency of $300 \mathrm{~lm} / \mathrm{W}$, and a circle of $6 \mathrm{~mm}$ in diameter, a total output from the phosphor of 376,000 lumens can be obtained, allowing projectors to have screen lumens in the 120,000 screen lumens ranges. Again, further power increase can be achieved by further increase in outer diameter while the etendue maintains to be the same as that of the $2 \mathrm{~mm}$ spot, which is only $\mathrm{E}=9.87$.

\begin{tabular}{c|c|c|c|}
\hline $\begin{array}{c}\text { Current } \\
(\mathrm{A})\end{array}$ & $\begin{array}{c}\text { BLD Input } \\
(\mathrm{W} \text { oP })\end{array}$ & $\begin{array}{c}\text { Spot size } \\
(\mathrm{mm})\end{array}$ & $\begin{array}{c}\text { Power density } \\
\left(\mathrm{W} / \mathrm{mm}^{2}\right)\end{array}$ \\
\hline 0.5 A & 0.0300 & 0.48 & 0.166 \\
\hline 1.0 A & 5.4000 & 0.48 & 29.842 \\
\hline 1.5 A & 13.0800 & 0.48 & 72.283 \\
\hline 2.0 A & 20.6400 & 0.48 & 114.061 \\
\hline 2.5 A & 27.9600 & 0.48 & 154.513 \\
\hline 3.0 A & 34.9000 & 0.48 & 192.865 \\
\hline 3.5 A & 41.7800 & 0.48 & 230.885 \\
\hline 4.0 A & 48.3800 & 0.48 & 267.358 \\
\hline 4.5 A & 54.6000 & 0.48 & 301.731 \\
\hline
\end{tabular}

Figure 4 - Experimental Results

\section{Integrated White Light System}

Although the system as shown in Figure 3 produces high output, but the light is mostly yellow since the blue light is mostly filtered out at the output. If a true white light output is required, blue light has to be added separately or as an integrated system as shown in Figure 5. In this system, part of the input blue laser light is reflected towards a high temperature ceramic diffuser plate, e.g. $20 \%$, of which scanning is not needed. The balance of the blue light will be used for the rotating mirror system generating yellow light. The same blue reflector will combine the diffused blue light and the yellow light into a single white light output beam.

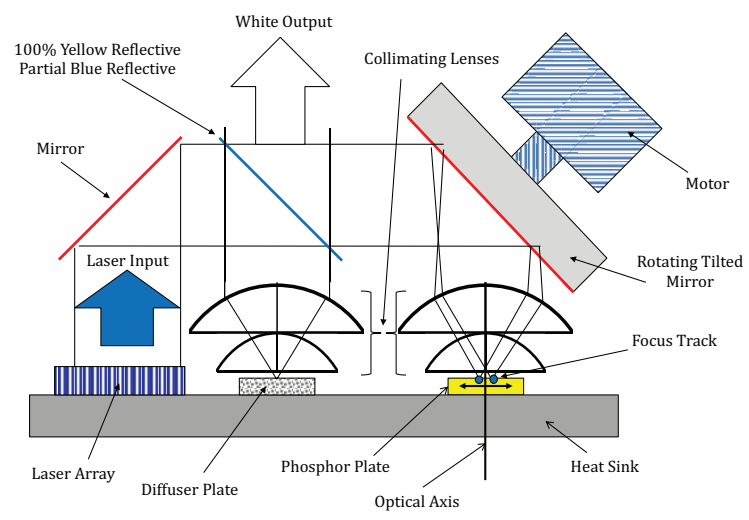

Figure 5 - Scanning Focus Beam Static Phosphor System with Integrated Blue Light

\section{Conclusion}

Using a compact patent pending OTI Tilted Rotating Mirror system, equivalent power density on the phosphor plate of over 6.7 times has been demonstrated. Further work is being perform increasing this power limitation and the integrating of blue light and yellow light with the same system. It is expected that cinema projectors with output in the range of 120,000 screen lumens coule become a reality. 
LDC2021 | Oral Presentation

\title{
Smart systems
}

Thu. Apr 22, 2021 1:00 PM - 2:30 PM LDC (Online)

Click to the Online Room

\author{
[LDC-9-01 (Invited)] Geo-Marine Science using Visible Light \\ OHiroshi Yoshida ${ }^{1}$, Shojiro Ishibashi ${ }^{1}$ (1.JAMSTEC) \\ 1:00 PM - 1:30 PM \\ [LDC-9-02] Imperceptible Projected Marker Codes with Application to \\ Calibration-Free Projection Mapping \\ OShingo Kagami ${ }^{1}$ (1.Tohoku University) \\ 1:30 PM - 2:00 PM \\ [LDC-9-03] \\ Scanning RGB Laser Beam Detection for Smart Laser Display \\ System \\ OTakeshi Ebara ${ }^{1}$, Hiroshi Murata ${ }^{1,2}$, Masato Ishino ${ }^{2}$, Junichi Kinoshita ${ }^{2}$, Kazuhisa \\ Yamamoto $^{2}$ (1.Mie University, 2.Institute of Laser Engineering, Osaka University) \\ 2:00 PM - 2:15 PM \\ [LDC-9-04] \\ Extraction Method of Typical Traffic Lines of Pedestrians \\ OAkinobu Watanabe $^{1}$ (1.Hitachi, Ltd.) \\ 2:15 PM - 2:30 PM
}


LDC2021 | Oral Presentation

Smart systems

Thu. Apr 22, 2021 1:00 PM - 2:30 PM LDC (Online)

\section{[LDC-9-01 (Invited)] Geo-Marine Science using Visible Light}

OHiroshi Yoshida ${ }^{1}$, Shojiro Ishibashi ${ }^{1}$ (1.JAMSTEC)

Exploration of natural resources in the region of seabed in Japan' s exclusive economic zone (EEZ) are needed for economic growth. Underwater ranging is also important to realize plate motion or crustal movement. These data measured are transmitted to a support ship. Acoustic imaging is traditionally used for underwater exploration, ranging and communication. Underwater laser provides us high resolution and more accurate data high-rate communication. JAMSTEC has developed and tested underwater laser scanners, underwater range finder and UWOC systems since 2009. 


\title{
Geo-Marine Science using Visible Light
}

\author{
Hiroshi Yoshida ${ }^{1}$, Shojiro Ishibashi ${ }^{2}$ \\ ${ }^{1}$ Japan Agency for Marine-Earth Science and Technology (JAMSTEC), \\ 2-15 Natsushima, Yokosuka, Japan, +81-46-867-938, yoshidah@jamstec.go.jp \\ ${ }^{2}$ JAMSTEC
}

\begin{abstract}
Exploration of natural resources in the region of seabed in Japan's exclusive economic zone (EEZ) are needed for economic growth. Underwater ranging is also important to realize plate motion or crustal movement. These data measured are transmitted to a support ship. Acoustic imaging is traditionally used for underwater exploration, ranging and communication. Underwater laser provides us high resolution and more accurate data high-rate communication. JAMSTEC has developed and tested underwater laser scanners, underwater range finder and UWOC systems since 2009.
\end{abstract}

\section{Introduction}

Underwater resources exploration and sea-floor mapping are important work for Japan. For observation of seafloor surface, side scan sonar, multi-beam echo sounder and camera are widely utilized. Sonars are suitable for imaging in long range of from a few tens meter to a few hundred meters. Underwater camera is well-known effective tool for close rang imaging. For mid-range (approximately $5 \mathrm{~m} \sim 30 \mathrm{~m}$ ) observation, high frequency sonar is commercialized and a laser imaging system which consists of a sheet laser and a CCD (charge coupling device) camera was developed by engineering group of University of Tokyo [1]. JAMSTEC has developed a 30 m-range laser scanning system used a high-power pulse laser and a PMT (photo multiplier tube) since 2011 based on previous research of underwater laser beam propagation [2]. We also developed and tested an underwater rangefinder and an underwater optical communication (UWOC) system. Sawa [4] achieved long-range optical wireless communication with rate of $20 \mathrm{Mbps}$ in range of $120 \mathrm{~m}$. In this paper we review underwater laser applications including scanning system, ranging system and communication system.

\section{Laser scanner, laser ranging, UWOC system}

Fig. 1 shows attenuation coefficients measured in Sagami-bay in depth between 0 and $500 \mathrm{~m}$ with an insitu laser propagation measurement tool or a beam transmittance meter with wavelength, $\lambda$, of $473 \mathrm{~nm}$ and $523 \mathrm{~nm}$. We estimated that propagation loss becomes 1 $\mathrm{dB} / \mathrm{m}$ or more by shadowing effect of marine snow because Sagami-bay is the sea where ocean nutrients are relatively increased. Attenuation is drastically decreased around $90 \mathrm{~m}$ in depth. When depth is more than $100 \mathrm{~m}$, attenuations of about $0.2 \mathrm{~dB} / \mathrm{m}(\lambda=473 \mathrm{~nm})$ and 0.5 $\mathrm{dB} / \mathrm{m}(\lambda=523 \mathrm{~nm})$ were obtained, respectively. These attenuation curves would strongly correlate with turbidity and chlorophyll-a. We also carried out attenuation experiments including the measurements near the sea floor. Most of data show that the attenuation in deep sea under 100 meters depth would be $0.5 \mathrm{~dB} / \mathrm{m}$ or less.
An underwater 3-D laser scanner (30 $\mathrm{m}$ range, $2 \mathrm{~cm}$ resolution) developed by JAMSTEC and Mitsubishi Electric TOKKI Systems Co. was obtained wide area high resolution bathymetry. The laser scanner was installed in an autonomous underwater vehicle "Otohime", which is $2.5 \mathrm{~m}$ long and $900 \mathrm{kgs}$, and deployed in Ohmura-dashi where is hydro-thermal plume area, in 2016. Fig. 2 shows an obtained bathymetry and a plume enlarged with resolution of 2 $\mathrm{cm}$. A camera captured image also shown for comparison.

We developed a prototype of an underwater laserranging system to detect the fluctuations for long-term prediction of oceanic intraplate earthquake as shown in Fig. 3. Experimental results show that measurement accuracy of the system was $1.5 \mathrm{~mm}$ at distance of $10 \mathrm{~m}$. It is poor result compared to the expected accuracy of $0.5 \mathrm{~mm}$ or less. The remaining tasks on the laser-ranging are: 1) extending measurement range and 2) developing a robust alignment system. We also need a high accuracy sea water permittivity measuring instrument to reduce measurement errors.

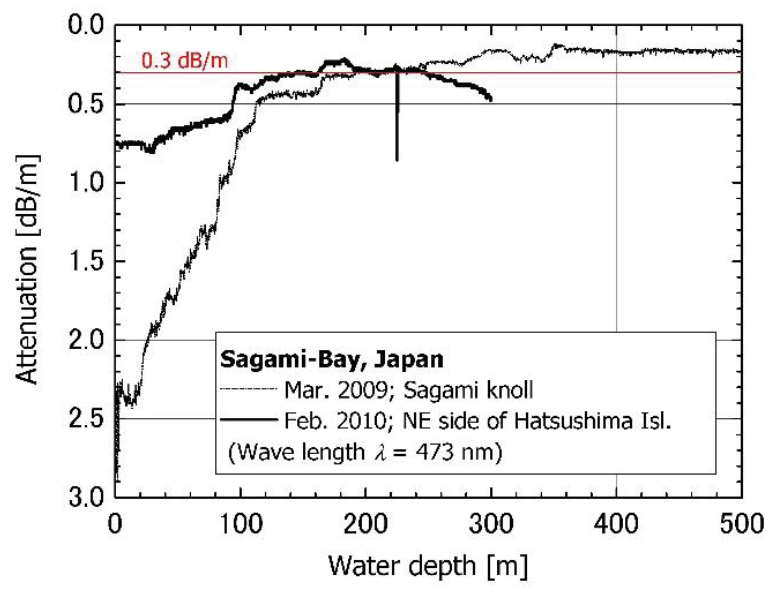

Fig. 1. An underwater light attenuation measured in March 2009, in Sagami knoll, Japan. 


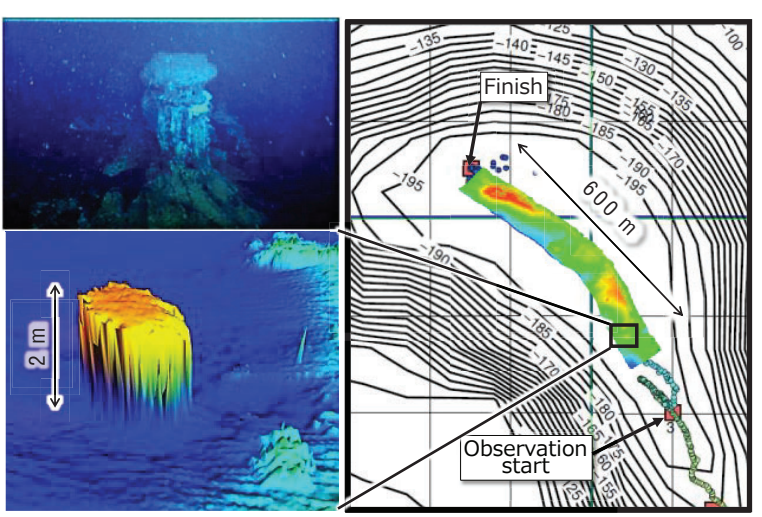

Fig. 2 Underwater laser imaging of sea-floor in Ohmurodashi, Japan.

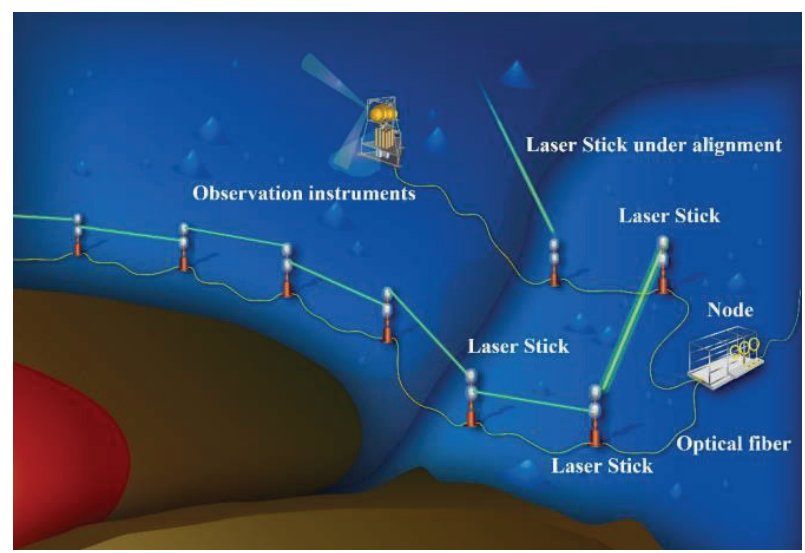

Fig. 3 Deployment image of an underwater laser-ranging for measurement of plate movement.
Underwater Optical communication studies are also performed in JAMSTEC. Sawas' group achieved deepsea laser bi-directional communication at depth of $700 \mathrm{~m}$ using the deep-sea ROV "Kaiko" [4]. This ROV consists of two part: a launcher and a vehicle. The both are connected with a tether cable. They installed one of transceiver in the launcher and the other in the vehicle. The vehicle was vertically deployed and adjusted optical axis by controlling thrusters. They achieved $20 \mathrm{Mbps}$ bidirectional communication in range of $120 \mathrm{~m}$.

\section{Concluding remarks}

Underwater laser technologies are introduced based on JAMSTEC's development system. The laser scanner and Sawa's UWOC system were commercialized. Now some researchers are conducting development new underwater laser system of an extended-range laser scanner and an ultrahigh-rate optical communication system. Evolution of underwater optical system knows no boundaries.

\section{References}

[1] Bodenmann, A., Thornton, B., Ura, T., Sangekar, M., Nakatani, T., Sakamaki, T., Pixel based mapping using a sheet laser and camera for generation of colored $3 \mathrm{D}$ seafloor reconstructions, Proc. OCEANS Conf. 2010.

[2] H. Yoshida, T. Saito, S. Ishibashi and S. Okamoto, "Basic study of underwater laser propagation," Proc. of 9th SEGJ International Symposium, Sapporo, p. 45, 2009.

[3] Shojiro Ishibashi, et al., "Seabed 3D images created by an underwater laser scanner applied to an AUV," Proc. OCEANS 2017, Anchorage, Sept. 2017.

[4] Takao SAWA, et al., "Practical Performance and Prospect of Underwater Optical Wireless Communication," IEICE Trans. Fundamentals, E102.A(1), pp. 156-167 (2019). 
LDC2021 | Oral Presentation

Smart systems

Thu. Apr 22, 2021 1:00 PM - 2:30 PM LDC (Online)

\section{[LDC-9-02] Imperceptible Projected Marker Codes with Application to Calibration-Free Projection Mapping}

OShingo Kagami ${ }^{1}$ (1.Tohoku University)

This paper reviews our recent efforts on Digital Micromirror Device (DMD)-based approaches to embed imperceptible marker codes into video projection. An application to calibration-free projection mapping onto a moving surface is also described. 


\title{
Imperceptible Projected Marker Codes with Application to Calibration-Free Projection Mapping
}

\author{
Shingo Kagami ${ }^{1}$ \\ ${ }^{1}$ Graduate School of Information Sciences, Tohoku University, 6-6-01 Aramaki Aza Aoba, Aoba-ku, Sendai, Japan \\ $\operatorname{swk}($ at)ic.is.tohoku.ac.jp
}

\begin{abstract}
This paper reviews our recent efforts on Digital Micromirror Device (DMD)-based approaches to embed imperceptible marker codes into video projection. An application to calibration-free projection mapping onto a moving surface is also described.
\end{abstract}

\section{Introduction}

Machine-readable marker codes such as 2D barcodes play various roles in many fields. Showing them on graphical displays is also a popular practice to provide additional information as well as the geometrical relationship between the display surface and a viewing device such as a camera. It is sometimes useful to make those codes imperceptible to human eyes such that they do not disturb the main displayed content. There are a wide range of techniques to achieve this, including the use of imperceptible optical properties [1-3], high temporal frequency components of signals [4-11], high spatial frequency components of patterns [12], insensibly small differences of luminance [13], and combinatory use of them.

A Digital Micromirror Device (DMD) is a spatial light modulator used in Digital Light Processing (DLP) projectors. Because a DMD is able to display fast flapping binary patterns at a rate of tens of $\mathrm{kHz}$, it is suitable for taking the high temporal frequency approach while relying on a mature and widely used type of display technology. This paper reviews the efforts in the author's group for expanding the possibilities and applications of imperceptible codes displayed with DMD projectors.

\section{Auto-synchronizing marker code}

A typical usage of a DMD display in embedding imperceptible information is to insert a short period of an embedded code frame periodically such that the period is short enough not to be visible to human eyes, and let the exposure time of a viewing device synchronized to those code frames $[5,6]$. If the synchronization can be acquired automatically, applications with multiple displays and multiple viewing devices, possibly owned by general public users, would be realized.

We proposed a technique that dynamically controls the frame rate of a camera such that the camera exposure period is automatically synchronized to displayed code frames [14]. It is an instance of a phase-locked loop (PLL) technique in which the pixel intensities of the marker code are received as input and the exposure control signal of the camera is generated as output [15]. A key idea is that the insertion period of the marker code frames into the displayed video is not perfectly constant. Instead, even-numbered code frames are inserted a little earlier, and odd-numbered frames a little behind. By analyzing the intensities of captured codes, it is possible to distinguish whether the camera capturing phase is in advance or behind compared to the code display phase.

\section{Synchronization-free marker code}

Another approach toward the same goal is to remove the necessity of synchronization. We developed a marker code embedding scheme in which small marker codes are sparsely distributed in space domain as well as in time domain within a displayed video [16].

Whenever a camera captures an image of the display surface with a sufficiently short exposure time, it is guaranteed that a small number of markers are detected. Each marker code provides information on where it should be in the display surface coordinates such that the camera is able to recover its geometrical pose with respect to the display surface coordinates. In the meanwhile, when we look at an arbitrary pixel in the display, it convey the pixel intensity of the main content for the most of the time and the insertion of marker codes occupies only a small fraction of the time.

\section{Application to calibration-free projection mapping} Projection mapping of video content onto a real object surface necessitates accurate registration between a projected image and the surface. When cameras are introduced to measure the surface geometry, accurate calibration between the projector and the cameras is required, which is in general a time-consuming task.

We proposed a calibration-free approach, in which the projected content as well as the surface is visually tracked with a camera, and a feedback control is applied such that the misalignment between them is minimized in real time. Implementation of this approach is relatively simple when tracking of the surface and the content is decoupled [17]. However, when a projected content is superimposed on the surface texture, decoupling the surface and the content in a camera image is challenging. When a movie content is projected, tracking the movie is also a challenge.

We applied the embedded-code approach to this problem [18], in which the proposed tracking algorithm is able to separate the projected code pattern and the surface texture from a single image without the need of time averaging or frame differencing of captured images, which will fail when the target surface moves rapidly. 
The algorithm is implemented with a DMD projector equipped with our custom controller [19-21], in which 400 -fps code patterns inserted within a 2,400-fps binary pattern sequence are tracked to achieve low-latency mapping.

\section{Conclusion}

This paper reported our recent efforts on improvement of DMD-based embedded-code techniques and application to dynamic projection mapping.

\section{Acknowledgment}

Part of this work was supported by JST ACCEL JPMJAC1601 and JSPS Grant-in-Aid 19H04146 and $16 \mathrm{H} 06536$.

\section{References}

[1] L. Zhang, N. Subramaniam, R. Lin, R. Raskar, and S. Nayar, "Capturing images with sparse informational pixels using projected 3D tags," In IEEE Virtual Reality Conference, pp.11-18. 2008.

[2] K. D. D. Willis, I. Poupyrev, S. E. Hudson, and M. Mahler, "SideBySide: Ad-hoc multi-user interaction with handheld projectors," In 24th ACM Symposium on User Interface Software and Technology (UIST2011), pp.431-440, 2011.

[3] S. Sakurai, Y. Kitamura, S. Subramanian, and F. Kishino, "A visibility control system for collaborative digital table," Pers. Ubiquitous Comput., Vol.13, No.8, pp.619-632, 2009.

[4] N. Matsushita, D. Hihara, T. Ushiro, S. Yoshimura, J. Rekimoto, and Y. Yamamoto, "ID Cam: A smart camera for scene capturing and ID recognition," In Second IEEE and ACM International Symposium on Mixed and Augmented Reality (ISMAR2003), pp.227-236. 2003.

[5] D. Cotting, M. Naef, M. Gross, and H. Fuchs, "Embedding imperceptible patterns into projected images for simultaneous acquisition and display," In 3rd IEEE/ACM International Symposium on Mixed and Augmented Reality (ISMAR2004), pp.100-109, 2004.

[6] I. E. McDowall, M. T. Bolas, P. Hoberman, and S. S. Fisher, "Snared illumination," In ACM SIGGRAPH International Conference and Exhibition on Computer Graphics and Interactive Techniques (SIGGRAPH 2004 Emerging technologies), p.24, 2004.

[7] A. Grundhöfer, M. Seeger, F. Hantsch, and O. Bimber, "Dynamic adaptation of projected imperceptible codes," In 6th IEEE and ACM International Symposium on Mixed and Augmented Reality (ISMAR2007), pp. 1-10, 2007.

[8] G. Woo, A. Lippman, and R. Raskar, "VRCodes: Unobtrusive and active visual codes for interaction by exploiting rolling shutter," In 2012 IEEE International Symposium on Mixed and Augmented Reality (ISMAR2012), pp.59-64, 2012.

[9] H. Yamamoto, S. Farhan, S. Motoki, and S. Suyama, "Development of 480-fps LED display by use of spatiotemporal mapping," In 2012 IEEE Industry Applications Society Annual Meeting, pp.1-7, 2012.

[10] G. Yamamoto, L. Sampaio, T. Taketomi, C. Sandor, H. Kato, and T. Kuroda, "Imperceptible on-screen markers for mobile interaction on public large displays," IEICE Trans. Inf. \& Syst., Vol.E100D, No.9, 2017.

[11] K. Jo, M. Gupta, and S. K. Nayar, "DisCo: Displaycamera communication using rolling shutter sensors," ACM Trans. Graph., Vol.35, No.5, 2016. Article No.150.

[12] T. Nakamura, A. Katayama, M. Yamamuro, and N. Sonehara, "Fast watermark detection scheme for cameraequipped cellular phone," In 3rd International Conference on Mobile and Ubiquitous Multimedia (MUM'04), pp.101-108, 2004.

[13] K. Kuraki, S. Nakagata, H. Ishii, M. Masui, and T. Anan, "Data transfer technology to enable communication between displays and smart devices," FUJITSU Science Technology, Vol.50, pp.40-45, 2014.

[14] H. Niidome, S. Kagami, and K. Hashimoto, "Camera synchronization to imperceptible frames embedded in a displayed video sequence," In 7th ACM SIGGRAPH Conference and Exhibition on Computer Graphics and Interactive Techniques in Asia (SIGGRAPH Asia 2014 Posters), 2014. Article No.25.

[15] L. Hou, S. Kagami, and K. Hashimoto, "Illuminationbased synchronization of high-speed vision sensors," Sensors, Vol.10, No.6, pp.5530-5547, 2010.

[16] T. Kusanagi, S. Kagami, and K. Hashimoto, "Lightning Markers: Synchronization-free single-shot detection of imperceptible AR markers embedded in a high-speed video display," In 16th IEEE International Symposium on Mixed and Augmented Reality (ISMAR2017 Adjunct), pp.229234, 2017.

[17] S. Kagami and K. Hashimoto, "Sticky Projection Mapping: 450-fps tracking projection onto a moving planar surface," In 8th ACM SIGGRAPH Conference and Exhibition on Computer Graphics and Interactive Techniques in Asia (SIGGRAPH Asia 2015 Emerging Technologies), 2015. Article No.23.

[18] S. Kagami and K. Hashimoto, "Animated Stickies: Fast video projection mapping onto a markerless plane through a direct closed-loop alignment," IEEE Trans. Vis. Comput. Graph., Vol.25, No.11, pp.3094-3104, 2019.

[19] S. Kagami and K. Hashimoto, "A full-color single-chipDLP projector with an embedded 2400-fps homography warping engine," In 45th ACM SIGGRAPH International Conference and Exhibition on Computer Graphics and Interactive Techniques (SIGGRAPH 2018 Emerging Technologies), 2018. Article No.1.

[20] S. Kagami, K. Higuchi and K. Hashimoto, "Puppeteered Rain: Interactive illusion of levitating water drops by position-dependent strobe projection," In 46th ACM SIGGRAPH International Conference and Exhibition on Computer Graphics and Interactive Techniques (SIGGRAPH 2019 Posters), 2019. Article No.48.

[21] S. Kagami and K. Hashimoto, "Interactive Stickies: Lowlatency projection mapping for dynamic interaction with projected images on a movable surface," In 47th ACM SIGGRAPH International Conference and Exhibition on Computer Graphics and Interactive Techniques (SIGGRAPH 2020 Emerging Technologies), 2020. Article No.16. 
LDC2021 | Oral Presentation

Smart systems

Thu. Apr 22, 2021 1:00 PM - 2:30 PM LDC (Online)

\section{[LDC-9-03] Scanning RGB Laser Beam Detection for Smart Laser Display System}

OTakeshi Ebara ${ }^{1}$, Hiroshi Murata ${ }^{1,2}$, Masato Ishino $^{2}$, Junichi Kinoshita ${ }^{2}$, Kazuhisa Yamamoto ${ }^{2}$ (1.Mie University, 2.Institute of Laser Engineering, Osaka University)

Interruption of scanning RGB laser beams was detected by use of a high-speed photodiode and high-speed oscilloscope to check a response time for controlling smart laser display operation. Clear electrical pulse train signals were obtained from the photodiode. These results are useful for safety control of smart laser display systems. 


\title{
Scanning RGB Laser Beam Detection for Smart Laser Display System
}

\author{
Takeshi Ebara $^{1)} \quad$ Hiroshi Murata ${ }^{1), 2)} \quad$ Masato Ishino $^{2)} \quad$ Junichi Kinoshita $^{2)} \quad$ Kazuhisa Yamamoto $^{2)}$ \\ 1) Graduate School of Engineering, Mie University 1577 Kurima-Machiya-Cho, Tsu-City Mie 514-8507 Japan. \\ Phone: +81-59-231-9403 Fax:+81-59-231-9403 E-mail murata@elec.mie-u.ac.jp \\ 2) Institute of Laser Engineering, Osaka University 2-6 Yamadaoka, Suita, Osaka 565-0871 Japan.
}

\begin{abstract}
Interruption of scanning RGB laser beams was detected by use of a high-speed photodiode and high-speed oscilloscope to check a response time for controlling smart laser display operation. Clear electrical pulse train signals were obtained from the photodiode. These results are useful for safety control of smart laser display systems.
\end{abstract}

\section{Introduction}

Display/lighting systems using visible-wavelength semiconductor lasers have been attracting a lot of attention in contemporary photonics research fields owing to the wide colour gamut, high colour reproduction range, high brightness, simple and compact optical circuits, and low power consumption. These advantages are due to the semiconductor laser's unique characteristic as a light source compared to conventional light sources; a narrow optical spectrum width $(\sim \mathrm{nm})$, high spatial and temporal coherence, and light amplification in a small optical cavity $(<1 \mathrm{~mm})$ by stimulated emission. Recently, several advanced displays and lighting systems utilizing RGB semiconductor lasers have been developed and come into the market [1].

One important visible laser application is 'smart laser-display' based on a laser-beam-scanning system [1], [2]. In LDC 2020, we reported on high-speed control of RGB semiconductor lasers with arbitrary signal patterns to realise advanced high-resolution smart laser-displays for $2 \mathrm{~K}, 4 \mathrm{~K}$ and $8 \mathrm{~K}$ [3]. In this paper, experimental results about interruption and detection of scanning RGB laser beams are reported. By use of a simple beam detection circuit with a high-speed photodiode, useful information about laser beam scanning/driving operations and safety controls are obtainable, which is due to high quality optical beam characteristics from laser light sources.

\section{Experimental set-up}

The experimental set-up for interruption and detection of scanning RGB laser beams is shown in Fig. 1.

Semiconductor laser modules with a pig-tailed single-mode fibre for red/green/blue light were installed in the temperature-controlled block. The emission light wavelength values and operational current values for RGB lasers used in the experiments are summarized in Table 1. During the experiment, the temperature of each laser module was set at 20 degrees Centigrade. Fibre cables from the RGB laser modules were connected to a
RGB laser beam combiner. The combined light was fed to collimator lens to form an arbitrary colour beam.

The generated laser beam was injected to a MEMS mirror with an oscillation frequency of $\sim 6 \mathrm{kHz}$. Measured characteristics of the MEMS mirror used in the experiment are summarized in Fig. 2. Then, the coloured laser beam was projected on a screen to generate test patterns as shown in Fig. 1. The distance from the MEMS mirror to the screen and the PD was about $800 \mathrm{~mm}$.

To check a laser beam projection on the screen, a high-speed ( $\sim 1 \mathrm{GHz})$ Si photodiode (PD) with a bias-tee circuit was installed in the front of the screen. A signal from the high-speed PD was monitored by use of a digital oscilloscope with a frequency bandwidth of $\sim 4$ $\mathrm{GHz}$.

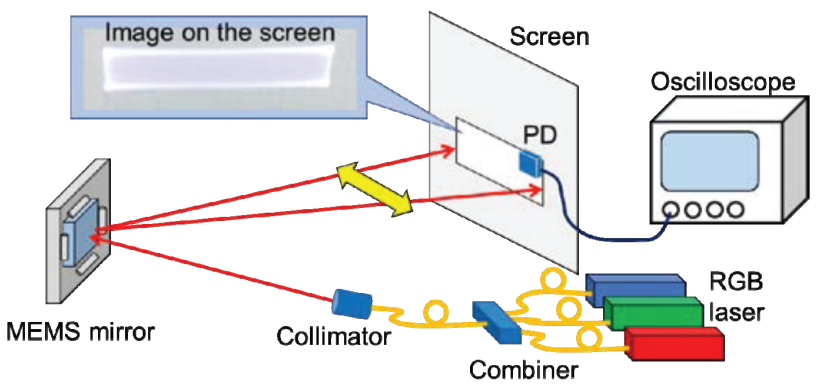

Fig. 1. Experimental set-up.

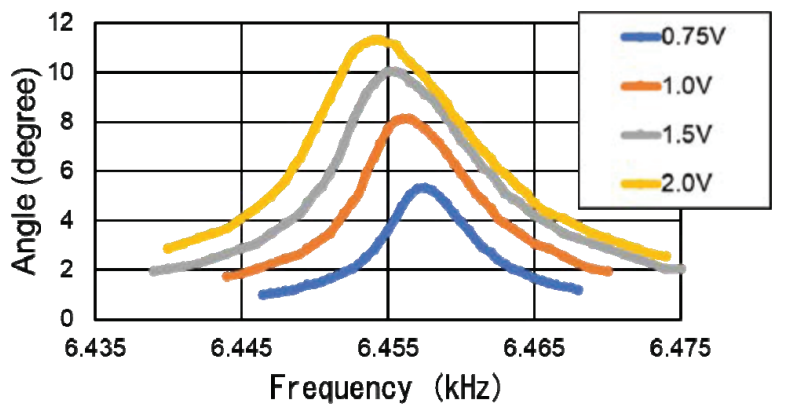

Fig. 2. Measured beam scanning characteristics of the MEMS mirror. 
Table 1. Parameters of the RGB lasers.

\begin{tabular}{c|c|c|c}
\hline & Red & Green & Blue \\
\hline $\begin{array}{c}\text { Operational wavelength } \\
\lambda(\mathrm{nm})\end{array}$ & $\sim 643$ & $\sim 518$ & $\sim 452$ \\
$\begin{array}{c}\text { Threshold current } \\
I_{\text {th }} \text { (mA) } \\
\begin{array}{c}\text { DC bias current } \\
I_{b}(\mathrm{~mA})\end{array}\end{array}$ & $\sim 55$ & $\sim 40$ & $\sim 21$ \\
\hline
\end{tabular}

\section{Results and discussion}

Typical examples of the measured signal from the highspeed PD are shown in Figs. 3 and 4. Clear pulse signals were observed according to the beam scanning frequency and the position of the PD as shown in Fig. 3. These measurements were repeated for red, green, blue, and white light cases by changing operational currents for the RGB laser modules. The obtained pulse signal widths were almost the same in these measurements.

In Fig. 4, the rise time and fall time of the detected pulse signal were $\sim 100 \mathrm{~ns}$, which is short enough to operate interlocking circuit for safety operation in smart laser display systems, we believe.

The measurement was also carried out when the light sources were replaced with a small commercially available laser scanning projector with a luminous flux of $\sim 20 \mathrm{~lm}$. When the PD was set at the position of $1,000 \mathrm{~mm}$ from the projector, clear pulse signals were also obtained.

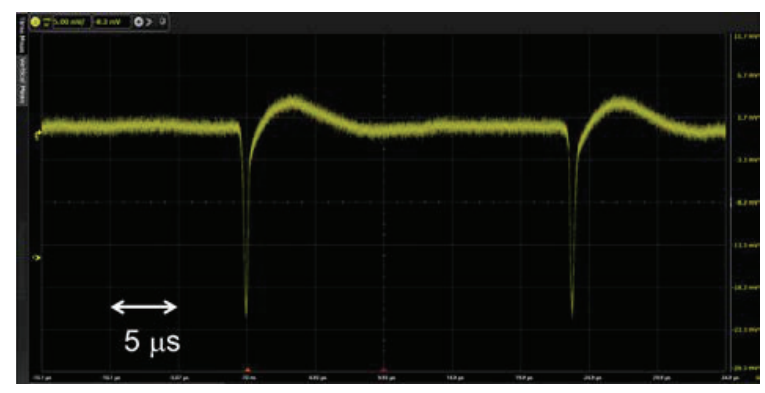

Fig. 3. Pulse signal trains from the photodiode.

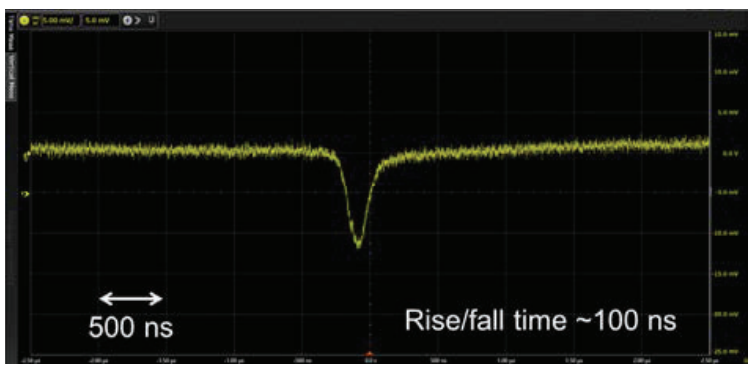

Fig. 4. Pulse signal from the photodiode with a high temporal resolution.

\section{Conclusions}

Basic measurement results for safety control of smart laser displays are reported. Rather clear pulse signals can be obtained from a standard PD when the PD was set on the front of a screen of $\sim 1 \mathrm{~m}$ apart from laser sources, which was due to the straight propagation with small diffraction of a laser beam, we believe. Details will be presented in the conference.

\section{Acknowledgment}

The authors thank Mr. Kiyoshiro Yamanaka for his help in the experiments. The authors are also grateful to support of Strategic International Standardization, Ministry of Economy, Trade and Industry, Japan.

\section{References}

[1] K. Kuroda, K. Yamamoto, and S. Kurimura, "Laser Display -from basics to applications-," ed. Optronics, Tokyo, Japan (2010).

[2] W. O. Davis, R. Sprague, and J. Miller, "MEMS-based pico projector display," Proc. 2008 IEEE/LEOS International Conference on Optical MEMs and Nanophotonics, Aug. 2008, Freiburg, Germany.

[3] K. Yamanaka, H. Murata, J. Kinoshita, and K. Yamamoto, "High-Frequency Digital Modulation of RGB Semiconductor Lasers for High-Resolution Scanning Displays," LDC 2020, April 2020, Yokohama, Japan. 
LDC2021 | Oral Presentation

Smart systems

Thu. Apr 22, 2021 1:00 PM - 2:30 PM LDC (Online)

\section{[LDC-9-04] Extraction Method of Typical Traffic Lines of Pedestrians}

OAkinobu Watanabe ${ }^{1}$ (1.Hitachi, Ltd.)

We developed the extraction method of typical traffic lines of pedestrians captured by TOF sensor, and grouping method of similar pathways with the typical traffic lines. 


\title{
Extraction Method of Typical Traffic Lines of Pedestrians
}

\author{
Akinobu Watanabe ${ }^{1}$ \\ ${ }^{1}$ Hitachi, Ltd. , Research \& Development Group, \\ 1-280, Higashi-koigakubo Kokubunji-shi, Tokyo, 185-8601 Japan, \\ +81-80-2097-8607, akinobu.watanabe.fz@hitachi.com
}

\begin{abstract}
We developed the extraction method of typical traffic lines of pedestrians captured by TOF sensor, and grouping method of similar pathways with the typical traffic lines.
\end{abstract}

\section{Introduction}

There is a need to apply product layouts that are effective in improving sales in a timely manner by measuring the movement of visitors in retail stores and analyzing the relationship between product layout and sales. In addition, by measuring the flow of visitors in commercial facilities and analyzing the relationship between events in tenant stores and facilities and the number of customers, there is a need to quantitatively evaluate effective measures for attracting customers, improve the customer attraction rate of commercial facilities, and realize congestion mitigation and lively creation.

In order to respond to these needs, it is necessary to enable quantitative evaluation by correctly measuring the flow of people in stores and facilities, finding valuable information obtained by analysis of people flow, and indexing it. In addition, it is necessary to be able to change the behavior of visitors by measures such as changing in-store notices, changing guidance, and implementing events, take actions to control the flow of people, and as a result, confirm that the indicators will change as expected.

As we face the challenge of COVID-19, it is important to control the flow of people to a density that can suppress the occurrence of excessively dense conditions and maintain an appropriate distance between people in facilities where many people gather, such as commercial facilities and public facilities, in order to satisfy the peace of mind of visitors and to increase the number of visiting customers. In addition, automatic delivery robots and slow mobility for handicap people and the elderly are expected to become popular in the near future, and it is expected that the operation of mobility that requires location information to be grasped will become more common. Since the beginning, due to the increasing possession rate of smart devices such as smartphones, there is a tendency to increase the means of obtaining individual location information.

Even in such a world, as a mobile body that does not have position management functions, for example, there may be pedestrians and wheelchair users who do not possess smart devices, and environmental sensing type traffic line measurement means are expected to play an important role as one of the means to acquire the position information and interpolation other measurement means.

\subsection{Backgroud}

Extraction methods based on the similarity of the grid shape, extraction methods for sampling the main transit points in stores, and extraction methods along the transportation network, have been proposed as conventional mainline extraction methods [1-3].

However, in order to realize a line analysis solution for commercial facilities and retail stores, it is necessary to have a method suitable for the purpose of grasping the characteristics of the behavior of people entering and exiting through closed space entrances and exits such as retail stores and commercial facilities.

\subsection{Purpose}

The purpose of this study is to obtain a typical pattern of behavior from the flow of people entering and leaving a small closed space. To that end, we have developed a method to rank the traffic lines in order of many people following the same line of motion for the direction of motion.

\subsection{Method}

In this study, we hypothesis that the lines of movement followed by customers of retail stores are closely related to the purchasing behavior of customers, and acquire customer behavior as a line of motion. And in an environment where customers can assume the product layout in the store to some extent in advance, it is expected that a typical customer behavior pattern exists, and it will appear as a traffic line following the same path.

Then, based on the obtained customer's traffic line, it is confirmed that a characteristic tendency can be seen in the direction in which people flow for each place in the store. If the tendency of the flow of the person for each place is obtained, the degree whether the traffic line is a trajectory along the flow of the same person as the other traffic line is obtained for one traffic line as a score quantitatively numerical for each place from the start point of the traffic line to the end point. If the entire traffic line is a trajectory along the flow of each location, the score is higher, so it can be said that the traffic line with a high score shows typical behavior. 


\section{Experiment}

Specifically, abobe method was implemented as follows. First, in order to obtain the trend of the direction by location, the floor plan of the target store area was considered to be an XY plane, and it was divided into a square grid of equal size. Next, from a typical value of a person's shoulder width and aisle width, a circle on an $\mathrm{XY}$ plane with a radius of a certain length is set as the center of the grid as the center of the circle, and a traffic line passing through the grid in the circle is extracted for each grid. Cluster the orientation of the extracted traffic lines in the $\mathrm{N}$ direction and add them to the grid at the center of the circle. As a result, even a traffic line that does not exactly pass the same coordinates is considered to have passed the same coordinates, and the deviation between the traffic lines due to minute measurement errors, body differences, and wobbles of walking can be ignored. Further, as a feature amount of the flow of the field, it was assumed that clustering in 8 directions sufficiently represents the traffic line in the store composed of passages in the $\mathrm{X}$ direction and the $\mathrm{Y}$ direction. By adding the score in the same direction from all grids through which the traffic line passes, and by cracking it by the number of coordinates, the score of the traffic line is set, and by ranking with this traffic line score, the upper traffic line is acquired as the main traffic line.

As a method for determining the similarity line, the distance between the coordinate $\mathrm{P}$ at the traffic line $\mathrm{A}$ and the coordinate $\mathrm{Q}$ on the $1 \mathrm{st}$ traffic line $\mathrm{B}$ is acquired for the target line B comparing whether or not it is similar to the base typical traffic line A, and the smaller the total distance, the higher the similarity. Furthermore, the difference in the direction of the vectors of each traffic line A and B in coordinates $\mathrm{P}$ and coordinate $\mathrm{Q}$ is obtained in the entire traffic line, and the smaller the total of the direction difference, the higher the similarity. Coordinate $\mathrm{P}$ is a coordinate sampled at a distance relative to the stride of the traffic line A. This allows the stop to absorb the phenomenon of concentrating many sampling points at the same coordinates.

By combining the above, the similarity line group of the upper traffic line of the ranking is extracted, and the main traffic line is extracted based on the number of movement lines ranking and score ranking of each group.

By limiting the start point and end point in a fixed area, it is possible to exclude employee lines and split lines that are noise factors assuming visitors to small retail stores. On the other hand, if the employee's line of motion is targeted, evaluation is possible by setting the start and end points to areas that only employees can enter, such as cash register areas and backyards, and by obtaining a line of motion that excludes the customer's movement line from the entire group of traffic lines.

\section{Result}

In this study, 118 traffic lines were extracted from 3D point cloud data obtained by 3D LiDAR, which generates traffic line data, enters the store through an area equivalent to an entrance and exit, and takes a trajectory to pass through the same area and leave the store. As for the layout in the store, there are four passages in the $\mathrm{X}$ direction and two passages in the $\mathrm{Y}$ direction. In addition, there is a short passageway connecting the passageways and a space where you can eat and drink near the entrance. On the right, there is also a space where only clerks can enter. The aisles are separated by product shelves, and the outer part of the store is also surrounded by product shelves.Fig. 1. is a figure in which 118 traffic lines are visualized as lines on the XY plane.

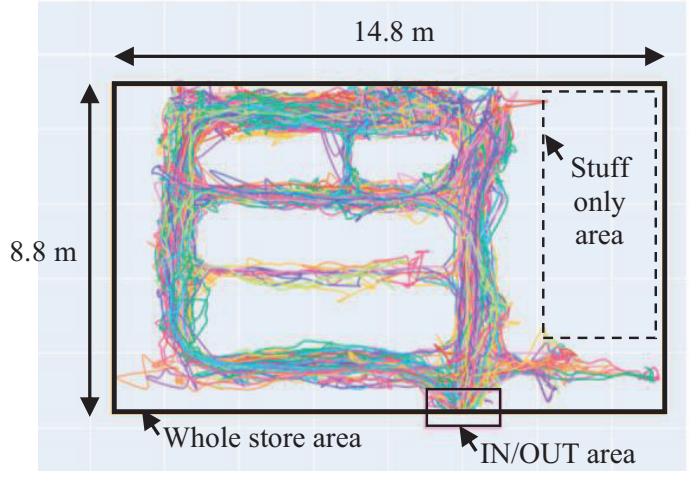

Fig. 1. All traffic lines in a retail store captured by 3D LiDAR

\subsection{Scoring line direction}

The vector showing the direction of the traffic line at all coordinates of the traffic line is clustered in 8 directions for each grid on the XY plane divided into $200 \mathrm{~mm}$ squares. Considering the shoulder width and aisle width, the clustered 8-way orientation distribution of the grid present in a circle with a radius of $800 \mathrm{~mm}$ is added as a distribution of the 8-way orientation of the grid at the center of the circle. Fig. 2. is a figure shown by a heat map with a distribution of orientations in eight directions as a feature amount of the field. The traffic line score is extracted by adding the number of clusterings in the direction that match the direction of the traffic line out of the number of clusterings in the eight directions of the grid through which each traffic line passes, and the calculated value of the entire traffic line is the number of coordinates that make up the traffic line as the traffic line score.
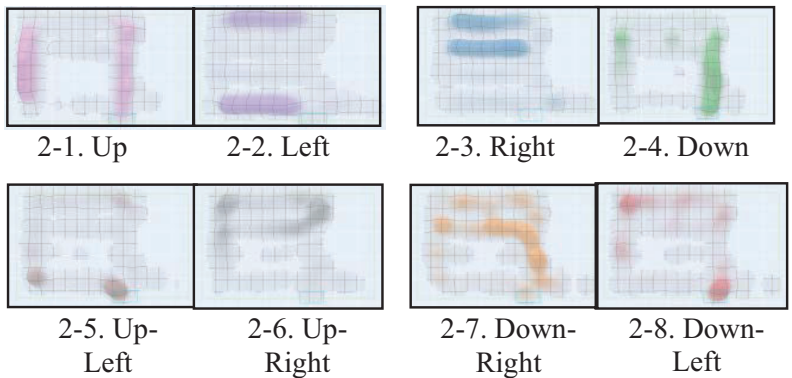

Fig. 2. Heatmap of 8 directions of traffic line. 
Fig. 3. is a figure showing the traffic line score of 118 traffic lines. The highest score is 1579 and the lowest score is -791 .

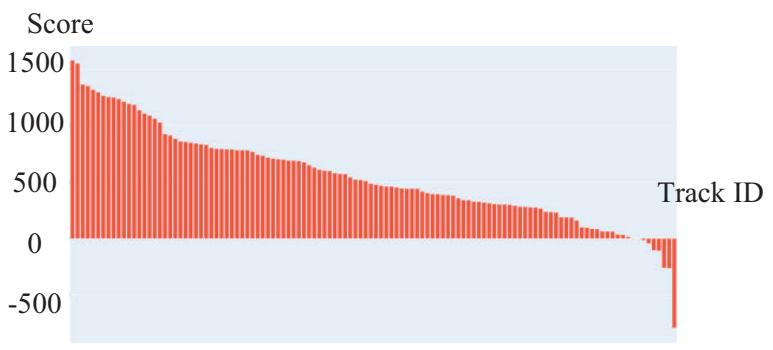

Fig. 3. Scores of all traffic lines.

\subsection{Similar lines extraction}

When grouping as a similar line, two patterns of 1000 $\mathrm{mm}$ and $1500 \mathrm{~mm}$ were evaluated as thresholds for the distance between the coordinate $\mathrm{P}$ that constitutes the traffic line A and the recent contact $\mathrm{Q}$ of the traffic line $B$ with value $P$ as value $S$, and as a threshold for the difference in the direction of the vectors of coordinates $\mathrm{P}$ and coordinate $\mathrm{Q}$, in two patterns of 90 degrees and 180 degrees as value $\mathrm{T}$. In addition, the moving distance to sample the traffic line A was evaluated in two patterns of $500 \mathrm{~mm}$ and $1000 \mathrm{~mm}$ as value U. Fig. 4. is a figure in which the group (the largest group) below the number of traffic lines that make up the group and the group with the highest average value of the traffic line score in the group (high score group) among the similar traffic line groups acquired under each condition are visualized as lines on the XY plane for each group.

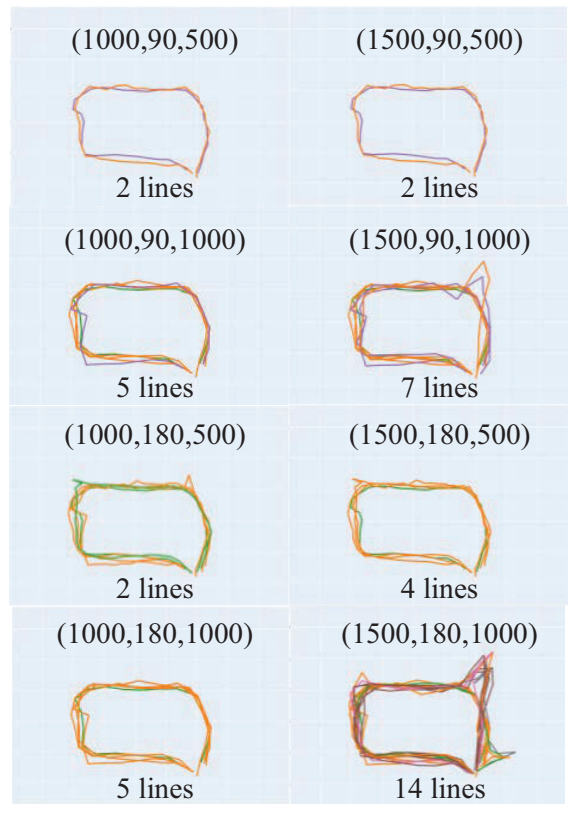

Fig. 4. Typical traffic lines for condition of (S, T, U)

\section{Discussion}

From the result of scoring line direction, the eight-way heat map shows the characteristics of the flow of people in each passage. It is natural that the lateral direction becomes the main flow of people in the passage in the $\mathrm{X}$ direction, and the longitudinal direction becomes the main flow of people in the passage in the $\mathrm{Y}$ direction. However, for example, the lower direction of figure 2-4. Down in Fig. 2. is strongly represented in the right passage, and it can be said that there is not much down flow in the left passage. Also, looking at figures 2-2. Left and 2-3. Right in Fig. 2, the left flow is strongly represented in the top and bottom passages, and the right flow is strongly represented in the first and second passages from the top.

From the result of similar lines extraction, $\mathrm{T}=90$ degrees and $\mathrm{T}=180$ degrees are compared as patterns that do not consider similarity due to direction difference. In the case of $\mathrm{T}=90$ degrees, it becomes a pattern considering the difference in orientation, and in addition to the coordinates, the movement line becoming a more similar traffic line if the direction of movement is the same. In the case of $\mathrm{T}=180$ degrees, it becomes a pattern that does not take into account the difference in direction, and as an extreme example, when the movement trajectory from entering to leaving the store compares the opposite movement line with each other, the similarity is high. It is possible to include or exclude the contribution of the difference in direction when such a case is included in the traffic line with a high similarity and when it is not included. In this data, there was no pair of traffic lines to draw the trajectory in the opposite direction, so there was no difference in the extraction result by considering the difference in direction or not.

$\mathrm{S}=1000 \mathrm{~mm}$ and $\mathrm{S}=1500 \mathrm{~mm}$ are compared as the threshold of the maximum value of the distance between the traffic lines to determine whether or not it is a similarity line. In the case of $\mathrm{S}=1000 \mathrm{~mm}$, when the distance between the traffic lines exceeds $1000 \mathrm{~mm}$ compared to the case of $\mathrm{S}=1500 \mathrm{~mm}$, it can be said that the condition is more severe because it is not regarded as a similarity line. Conversely, in the case of $\mathrm{S}=1500 \mathrm{~mm}$, the condition regarded as a similarity line is looser than in the case of $\mathrm{S}=1000 \mathrm{~mm}$, and it is expected that more traffic lines are extracted as similarity lines. As a result, if it is $U=500 \mathrm{~mm}$, contrary to expectation, even in the case of $\mathrm{S}=1500 \mathrm{~mm}$, the same number of traffic lines as in the case of $\mathrm{S}=1000 \mathrm{~mm}$ is extracted as a similarity line. For example, when comparing the case of $(\mathrm{S}, \mathrm{T}, \mathrm{U})$ $=(1000,90,500)$ and $(S, T, U)=(1500,90,500)$, two similar traffic lines are extracted. However, if $U=1000$ $\mathrm{mm}$, there is a difference in the extraction result. That is, since the trajectory of the two patterns is extracted as one similar line group, it is considered that the condition is too loose.

The difference in the extraction result by the moving distance $U$ when sampling the coordinates on the traffic line by the moving distance is shown. For example, when comparing the case of $(S, T, U)=(1000,90,500)$ 
and $(S, T, U)=(1000,90,1000)$, the former extracts two similar lines and the latter extracts 5 similar lines. As a result, since it is not a different result as a main traffic line, it is considered that it is sufficient in the case of $U=$ $1000 \mathrm{~mm}$, where the number of coordinates to be evaluated is small and the processing load is expected to be lighter.

From the above, as an index for extracting the main traffic line, if it is a condition that uses the distance between the traffic lines and the difference in direction, it is expected that it can be extracted without problems. In addition, it was confirmed that the extraction accuracy deteriorated if the distance threshold between the traffic lines and the distance of sampling were taken too large without considering the direction difference. The threshold condition is shown like that the value $(\mathrm{S}+\mathrm{U})$ should not be greater than $2000 \mathrm{~mm}$.

\section{Conclusions}

We have developed a main line extraction method suitable for the application of grasping the characteristics of the behavior of people entering and exiting through small closed space entrances and exits.

As the next step, we evaluate much more large traffic line data with this method and brush up the threshold parameters, and study ohter method to extract other features from traffic line, for example lively of fields.

\section{References}

[1] A. Asahara, Y. Teramoto, K. Maruyama and R. Shibasaki, "Representative Path Extraction with Spatio-temporal Network Analysis", IPSJ Journal, 54(1), 135-145 (2013).

[2] A. Asahara, K. Maruyama and A. Sato, "Pedestrian Trajectory Analysis Based on Mixed Markov Model", IPSJ Journal, 52(1), 187-196 (2011).

[3] T. Koiso, K. Hattori, T. Yoshida and N. Imasaki, "Behavior Analysis of Customers and Sales Clerks in a Home Appliance Retail Store Using a Trail Analyzing System", IPSJ SIG Technical Report, 115(2003-UBI-002), 61-66 (2003). 
LDC2021 | Oral Presentation

\section{Novel and Emerging Technologies}

Thu. Apr 22, 2021 2:45 PM - 3:15 PM LDC (Online) Click to the Online Room

[LDC-10-01] Design an Extreme Ultraviolet Illumination System with High Uniformity OYanqiu Li ${ }^{1}$, Qian Hao ${ }^{1}$, Ke Liu ${ }^{1}$, Xu Yan ${ }^{1}$ (1.Beijing Institute of Technology) 2:45 PM - 3:00 PM

[LDC-10-02] Colorization of Arc3D using Projector Lighting Olkuya Saji ${ }^{1}$, Masafumi Nakata ${ }^{2}$, Yasuhiro Kashihara ${ }^{2}$, Atsushi Hayashi ${ }^{2}$, Hirotsugu Yamamoto $^{1,3}$ (1.Utsunomiya University, 2.NSC Co., Ltd., 3.JST, ACCEL) 3:00 PM - 3:15 PM 
LDC2021 | Oral Presentation

Novel and Emerging Technologies

Thu. Apr 22, 2021 2:45 PM - 3:15 PM LDC (Online)

\section{[LDC-10-01] Design an Extreme Ultraviolet Illumination System with High Uniformity}

OYanqiu Li ${ }^{1}$, Qian Hao ${ }^{1}$, Ke Liu' ${ }^{1}$ Xu Yan ${ }^{1}$ (1.Beijing Institute of Technology)

In this paper, an extreme ultraviolet illumination system matching an NA 0.33 projection objective is designed. Simulation results show that this illumination system can achieve high uniformity on the mask plane under different illumination modes. 


\title{
Design an Extreme Ultraviolet Illumination System with High Uniformity
}

\author{
*Yanqiu $\mathrm{Li}^{1}$, Qian $\mathrm{Hao}^{1}$, Ke Liu ${ }^{1}$ and $\mathrm{Xu} \mathrm{Yan}{ }^{1}$ \\ ${ }^{1}$ Key Laboratory of Photoelectronic Imaging Technology and System of Ministry of Education of China, School of Optics and \\ Photonics, Beijing Institute of Technology, Beijing 100081, China, phone: +86-010-6891-2946 \\ liyanqiu@bit.edu.cn
}

\begin{abstract}
In this paper, an illumination system matching an NA 0.33 projection objective is designed. The assignment of field facets and pupil facets under different illumination modes are obtained by the method based on combinatorial optimization. Results show that this illumination system can achieve high uniformity on the mask under different illumination modes. Key words: Extreme Ultraviolet Lithography, Illumination System.
\end{abstract}

\section{Introduction}

Extreme ultraviolet (EUV) lithography objectives with NA 0.33 are applied in industrial exposure tools to realize a resolution of $13 \mathrm{~nm}$ [1]. Image quality of EUV exposure tool is determined by the objective and performance of the illumination system. Illumination systems with fly's eye (composed of field facets and pupil facets) are chosen to illuminate the mask with high uniformity and realize different illumination modes [2]. By adjusting assignment relationships of field facets and pupil facets, illumination systems can achieve different illumination modes on the exit pupil. Some assignment methods have been proposed in recent years [3-5]. Among them, Jiang's method based on combinatorial optimization could obtain the optimal assignment relationship of field facets and pupil facets under a specific illumination mode automatically and efficiently [5]. However, the illumination area of Jiang's system is rectangular, while that of NA 0.33 isomorphic objective is arc-shaped. In this paper, the assignment method based on combinatorial optimization is applied to realize the design of an NA0.33 illumination system with a large arc-shaped field of view (FOV).

\section{Method}

To improve the resolution, illumination systems should enable to realize different illumination modes. Different illumination modes cause different distributions of pupil facets that participate in the illumination. The field facets and corresponding pupil facets must tilt to ensure each illumination channel's image superimposes at the same position of mask plane.

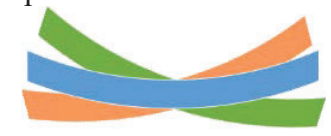

Fig.1. Schematic diagram of different tilts of different illumination channel's images on the mask plane.

Different assignment strategy leads to different tilt angles of different facet mirrors, and result in different tilt degrees of the illumination channel's image. The expected image of each illumination channel is depicted in blue as in Fig.1. But under some cases, the illumination channels' image may heavily deviate from the expected position, as depicted in green and orange, which deteriorate the illumination uniformity on the mask. To avoid this deterioration, tilts of illumination channel's images should be as small as possible.

Each field facet could assign to each pupil facet, and each pupil facet could receive the light from each field facet under a specific illumination mode. One field facet will have different tilt angles when assigned to different pupil facets, so the tilt of its corresponding image on the mask plane will be different. One pupil facet will also cause a different tilt of the illumination channel's image on the mask plane when receives rays from different field facets. Thus, there should be an assignment strategy that makes tilts of illumination channels' images on the mask as small as possib] ' 冫h can be solved by KuhnMunkres algorithm prop y Harold Kuhn [6,7].

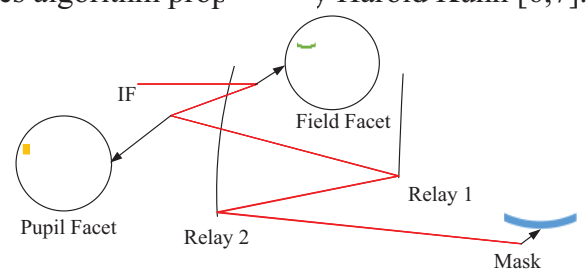

Fig.2. Real ray tracing of the illumination system.

To solve the problem, the cost of different assignment strategies should be calculated. The tilt angle of illumination channel's image, which taken as the cost, can be obtained by real ray tracing as in Fig. 2. Rays from the intermediate focus (IF) to the edges of the field facet under each mapping case are traced. Coordinates of the intersection points of the rays with the mask are acquired as $\left(x_{1}, y_{1}\right)$ and $\left(x_{2}, y_{2}\right)$, and then the tilt angle of each illumination channel's image under a specific mapping relationship can be calculated,

$$
\text { tilt }_{i j}=a \tan \left(\frac{y_{2}-y_{1}}{x_{2}-x_{1}}\right)
$$

After tilt angles under all possible mapping cases are obtained, the cost matrix can be established as in Fig. 3.

\begin{tabular}{|c|c|c|c|c|} 
& PFM1 & PFM2 & $\ldots \ldots$. & PFMn \\
\hline FFM1 & Tilt11 & Tilt12 & $\ldots \ldots$. & Tilt1n \\
\hline FFM2 & Tilt21 & Tilt22 & $\ldots \ldots$ & Tilt2n \\
\hline$\ldots \ldots$. & $\ldots \ldots$. & $\ldots \ldots$. & $\ldots \ldots$ & $\ldots \ldots .$. \\
\hline FFMn & Tiltn1 & Tiltn2 & $\ldots \ldots$. & Tiltnn \\
\hline
\end{tabular}

Fig.3. The cost matrix of the assignment problem. 
Finally, the Kuhn-Munkres algorithm is used to automatically determine the best assignment relationship.

\section{Results}

The initial configuration of the illumination system matching an NA0.33 projection objective is designed by the method in Ref. [5]. Table 1 shows the parameters of the NA0.33 objective. Fig. 4 shows the layout of the matching illumination system. Fig.5 shows the distribution of field facets and pupil facets, which are all arranged in a near circle contour.

Table 1. Parameters of the isomorphic projection objective

\begin{tabular}{c|c}
\hline Items & Value \\
\hline Numerical Aperture & 0.33 \\
Wavelength & $13.5 \mathrm{~nm}$ \\
Magnification & $1 / 4(\mathrm{X}) \times 1 / 4(\mathrm{Y})$ \\
Object field & $104 \mathrm{~mm} \times 8 \mathrm{~mm}$ \\
\hline
\end{tabular}

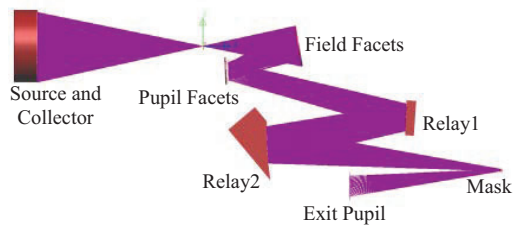

Fig. 4. Layout of the NA0.33 illumination system in LightTools.

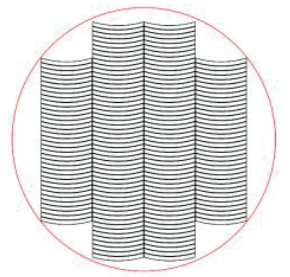

(a)

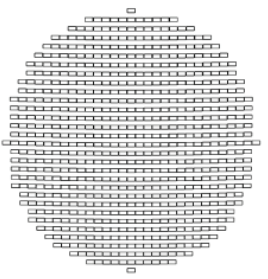

(b)
Fig. 5. Facets distribution of (a) field facets and (b) pupil facets.

Usually, the illumination uniformity on the mask plane of EUV illumination system is evaluated by:

$$
\text { Uniformity }=\left(1-\frac{I_{\max }-I_{\min }}{I_{\max }+I_{\min }}\right) \times 100 \%
$$

where $I_{\max }$ and $I_{\min }$ represent maximum and minimum light intensity in the scanning direction respectively.

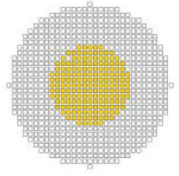

(a)

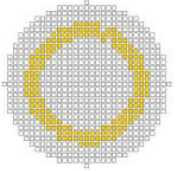

(b)

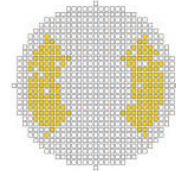

(c)

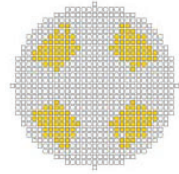

(d)
Fig. 6. Pupil facets' distribution under (a)conventional,

(b) annular, (c)dipole, and (d) quasar modes.

The illumination uniformity under four illumination modes have been evaluated. Fig. 6 shows the distribution of pupil facets that participate in the illumination under each illumination mode. The illumination uniformity under each illumination mode is simulated in LightTools and calculated by Eq. (2). Table 2 shows parameters of illumination modes and corresponding illumination uniformity, in which $\sigma_{i n}$ is the inner partial coherence factor, $\sigma_{\text {out }}$ is the outer partial coherence factor and $\theta$ is the opening angle.

Table 2. Illumination modes and illumination uniformity

\begin{tabular}{c|c|c|c|c}
\hline Modes & $\sigma_{\text {in }}$ & $\sigma_{\text {out }}$ & $\theta$ & Uniformity \\
\hline Conventional & -- & 0.50 & -- & $98.92 \%$ \\
Annular & 0.55 & 0.75 & -- & $98.90 \%$ \\
Dipole & 0.45 & 0.85 & $0^{\circ}$ & $98.90 \%$ \\
Quasar & 0.45 & 0.85 & $45^{\circ}$ & $98.90 \%$ \\
\hline
\end{tabular}

The illumination area under each illumination mode is shown in Fig.7. Results show this illumination system can achieve high uniformity under the four off-axis illumination modes.

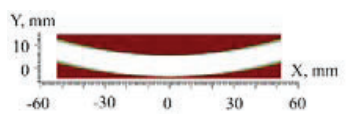

(a)

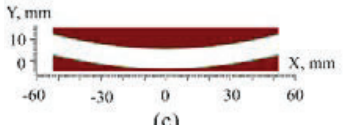

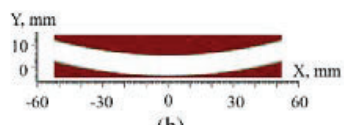

(b)

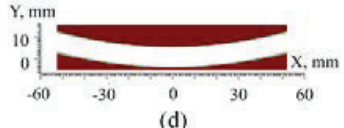

(d)
Fig. 7. Illumination area under (a)conventional, (b) annular, (c)dipole, and (d) quasar modes on the mask.

\section{Conclusion}

The assignment method based on combinatorial optimization is applied to realize the design of an NA0.33 illumination system with a large FOV. The facet mapping problem is formulated as an assignment problem and solved by Kuhn-Munkres algorithm. An initial configuration of the EUV illumination system matching an NA 0.33 objective with a large FOV is designed first. And facet assignment relationships of this system under different illumination modes are obtained by the assignment method described in this paper. Results show that this system can achieve high uniformity on mask under different illumination modes.

\section{References}

[1] M. Lowisch, P. Kuerz, and O. Conradi, "Optics for ASML's NXE:3300B platform,” Proc. SPIE 8679,86791H (2013).

[2] W. Singer, J. Schultz, and J. Wangler, "Illumination optics design for EUV lithography," Proc. SPIE 4146, 25-34 (2000).

[3] W. Singer, J. Schultz and J, Wangler, "Illumination system with variable adjustment of the illumination," U.S. Patent 2008/0225259 A1 (2008).

[4] T Fischer, "Illumination optical unit for EUV projection lithography," U.S. Patent 2015/0192860 A1 (2015).

[5] J. Jiang, Y. Li, S. Shen, and S. Mao, "Design of high numerical aperture extreme ultraviolet lithography illumination system," Appl. Opt. 57(20), 5673 (2018).

[6] B. Korte and J. Vygen, Combinatorial Optimization (Springer, 2012), Vol. 2.

[7] H. W. Kuhn, "The Hungarian method for the assignment problem," Nav. Res. Logist. Q. 2, 83-97 (1955). 
LDC2021 | Oral Presentation

Novel and Emerging Technologies

Thu. Apr 22, 2021 2:45 PM - 3:15 PM LDC (Online)

\section{[LDC-10-02] Colorization of Arc3D using Projector Lighting} Olkuya Saji ${ }^{1}$, Masafumi Nakata ${ }^{2}$, Yasuhiro Kashihara ${ }^{2}$, Atsushi Hayashi ${ }^{2}$, Hirotsugu Yamamoto ${ }^{1,3}$

(1.Utsunomiya University, 2.NSC Co., Ltd., 3.JST, ACCEL)

An autostereoscopic display by use of arc-shaped scratches, called Arc3D display, generates smooth motion parallax based on directional scattering. Arc3D on a glass substrate has been colorized and switched by use of a projector lighting. 


\title{
Colorization of Arc3D using Projector Lighting
}

\author{
Ikuya Saji ${ }^{1}$, Masafumi Nakata ${ }^{2}$, Yasuhiro Kashihara ${ }^{2}$, Atsushi Hayashi ${ }^{2}$, Hirotsugu Yamamoto ${ }^{1,3}$ \\ ${ }^{1}$ School of Engineering, Utsunomiya University, 7-1-2, Yoto, Utsunomiya, Tochigi, Japan 321-8585, \\ ${ }^{2}$ NSC Co., Ltd., 1-1-1, Tokura, Toyonaka, Osaka, Japan 561-0845, \\ ${ }^{3}$ JST, ACCEL, 7-1-2, Yoto, Utsunomiya, Tochigi, Japan 321-8585 \\ Email: hirotsugu@yamamotolab.science
}

\begin{abstract}
This paper describes the colorization of Arc3D, which is an autostereoscopic display by use of arc-shaped scratches on a transparent substrate. Directional scattering on an arc changes the position of an bright spot depending on the viewing position. Arc $3 D$ has been colorized and switched by use of a projector lighting.
\end{abstract}

\section{Introduction}

Recently, the prevalence of infectious diseases has increased interest in hygiene. One of the problems in daily life is the operation of buttons. In order to operate buttons without touching them, the display of buttons in the air and the sensing of pressing gestures are important technologies. Many types of button marks are used, and low-cost and highly flexible design is required for the aerial display of buttons. Arc3D is characterized by its low cost, smooth motion parallax, and wide viewing angle $[1,2]$. Conventional Arc3D is fabricated by using tools such as compass cutters, which is time-consuming. In addition, since the substrate needs to be disinfected and high chemical resistance is required for the substrate, we realized $\mathrm{Arc} 3 \mathrm{D}$ on a glass substrate using a pattern etching method.

In the case of button displays, it is necessary to change the color of the pressed button for applications such as elevator buttons. In this study, we describe the colorization of Arc3D using projector illumination.

\section{Principle}

\subsection{Principle of Arc3D}

The principle of Arc3D is shown in Fig. 1. An arcshaped scratch is made on a transparent substrate, and light is illuminated. Since the scattered light is directional, the position of the light spot on the arc is different for each eye. The position of the light spot on the arc differs between the two eyes because the scattered light is directional, and the light spot also moves as the viewpoint shifts. Thus, binocular disparity and motion parallax give depth to the aerial display.

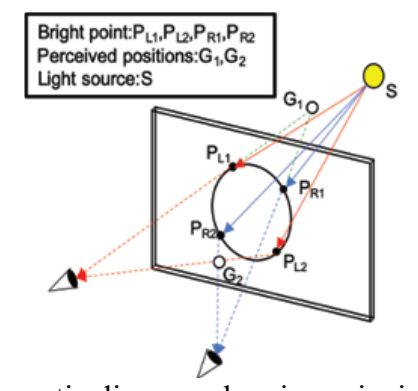

Fig. 1. Schematic diagram showing principle of Arc3D.
Since scattered light is visible within a limited range, only one bright spot is visible for each arc. The display content is composed by connecting these bright spots with many arcs. Fig. 2 shows Arc3D of the conventional method with collimated light illumination. The numbers 1 through 3 are displayed, and the radius of the arc is set to the radius corresponding to each number. In Arc3D, the pop-out distance varies with the radius.

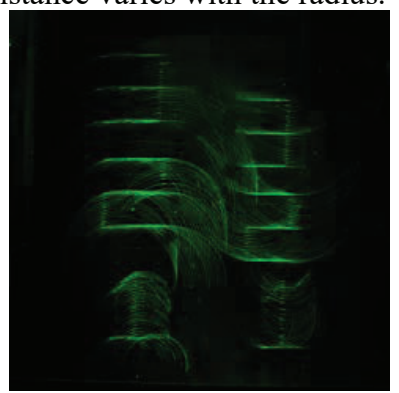

Fig. 2. Arc3D with conventional method.

\subsection{Arc3D by glass etching method}

Developed Arc3D by the pattern glass etching method is shown in Fig. 2. A mask pattern is used to etch circular arcs on a glass substrate, and many arcs can be etched at once, enabling mass-production at low cost. Compared with the conventional Arc3D method shown in Fig. 2, the detail of the bright spots and the inconspicuousness of the grooves were improved. This is due to the conditions of the groove depth and fineness, and the smoothness and uniformity of the grooves. By moving the viewpoint from left to right, we can see that the position of the bright spot is moving.

\section{Experiments}

The pattern-etched Arc3D was illuminated by a projector. The Arc3D was placed at the position where the screen was placed, and a part of the illumination range was applied to the Arc3D. The layout of the experiment is shown in Fig. 3. Four colors (red, blue, green, and yellow) were used for illumination, and the color display of Arc3D was divided into four sections. The illumination results are shown in Fig. 4. It can be seen that the color of the light spot changes depending on the color of the illumination. When no color is assigned, it is possible to turn off the display. 


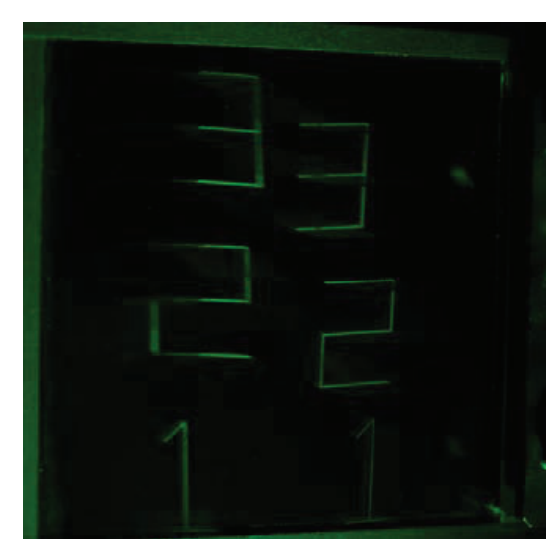

(a)

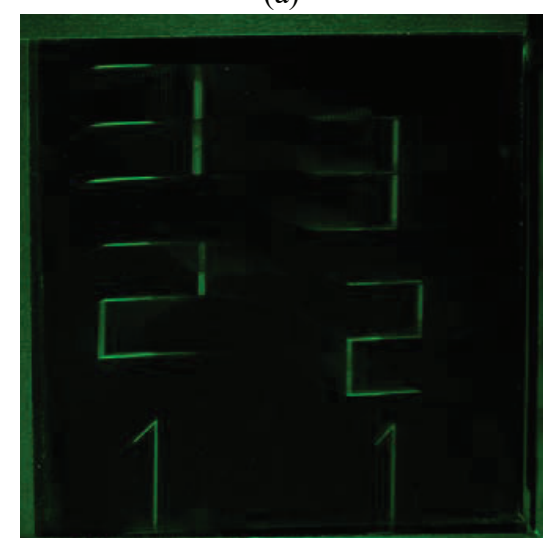

(b)

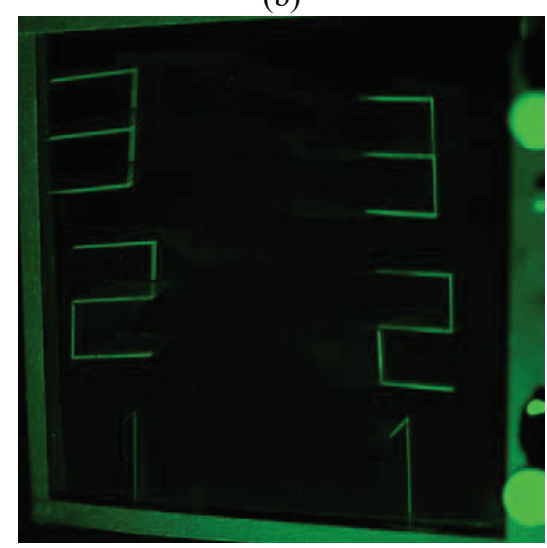

(c)

Fig. 3. Arc3D by pattern etching method. Viewed (a) from the left, (b) in front, (c) from the right.

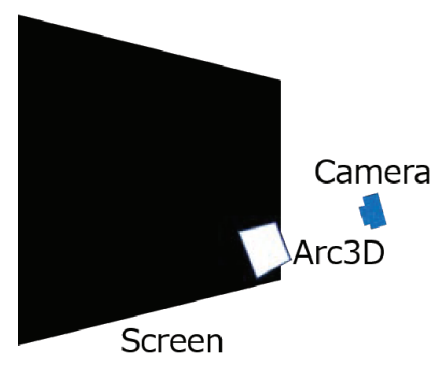

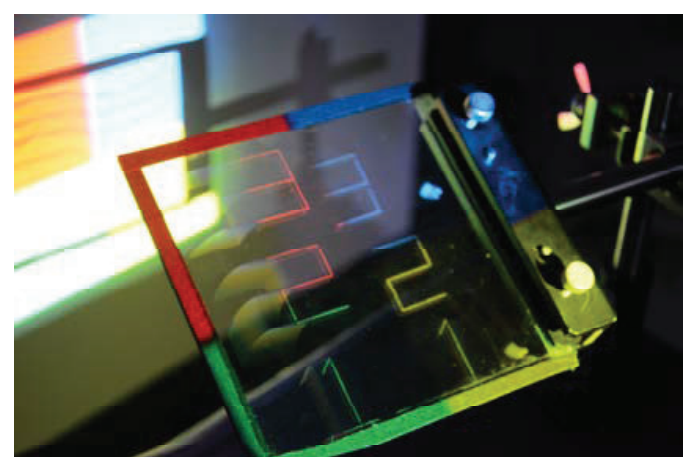

(a)

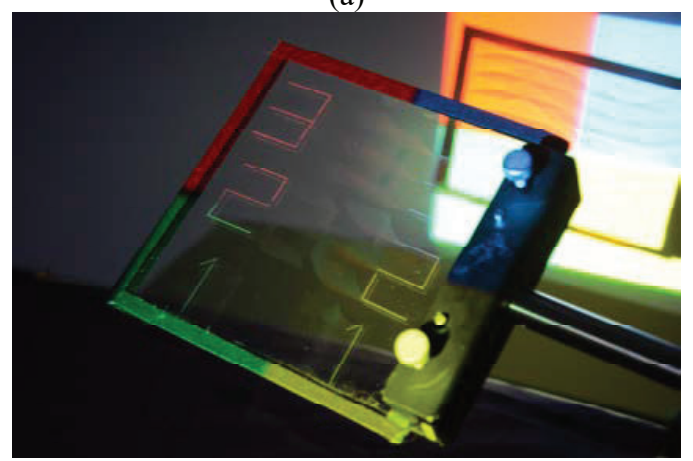

(b)

Fig. 4. Colorization of Arc3D using projector lighting. Viewed images (a) from the left, (b) from the right.

\section{Conclusion}

We have succeeded in changing the color of the Arc3D display in the specified color and range by irradiating the projector illumination. When applied to buttons, Arc3D can be produced at low cost by using glass substrate Arc3D, which has a high degree of freedom in design and excellent chemical resistance. Furthermore, since the color of the bright spot can be changed freely depending on the color of the illumination, it is possible to change the color when the button is pressed, turn the display on and off, and change the color depending on the type of button.

\section{Acknowledgment}

A part of this work was supported by JST/ACCEL (grant no. JPMJAC1601) and JSPS KAKENHI (19H04155, 20H05702).

\section{References}

[1] W. J. Beaty, "Drawing Holograms by Hand," Proc. SPIE 5005, 156-167 (2003).

[2] N. Yamada, C. Maeda, H. Yamamoto, and S. Suyama, "Theoretical and measured evaluation of lighting and observation angle dependence of perceived depth in arc 3D display," in The 19th International Display Workshop (IDW), 1219-1222 (2012).

Fig. 3. Experimental setups. 
LDC2021 | Oral Presentation

\title{
Closing Remarks
}

Thu. Apr 22, 2021 3:15 PM - 3:25 PM LDC (Online)

Click to the Online Room

\section{[LDC-Closing] Closing Remarks}

\author{
3:15 PM - 3:25 PM
}

Proc. of SPIE Vol. 11851 1185101-120 
3:15 PM - 3:25 PM (Thu. Apr 22, 2021 3:15 PM - 3:25 PM LDC)

\section{[LDC-Closing] Closing Remarks}

Proc. of SPIE Vol. 11851 1185101-121

Downloaded From: https://www.spiedigitallibrary.org/conference-proceedings-of-spie on 26 Apr 2023 Terms of Use: https://www.spiedigitallibrary.org/terms-of-use 